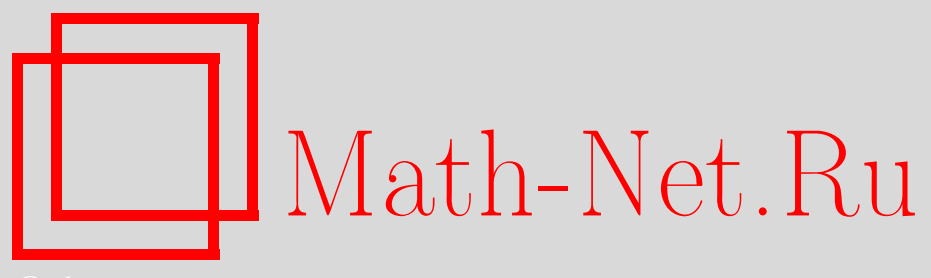

Е. И. Кайкина, П. И. Наумкин, И. А. Шишмарев, Асимптотика решений при больших временах для нелинейных уравнений типа Соболева, УМН, 2009, том 64, выпуск $3,3-72$

DOI: https://doi.org/10.4213/rm9299

Использование Общероссийского математического портала Math-Net.Ru подразумевает, что вы прочитали и согласны с пользовательским соглашением http: //www . mathnet.ru/rus/agreement

Параметры загрузки:

IP: 18.208 .226 .222

26 апреля 2023 г., 15:29:42

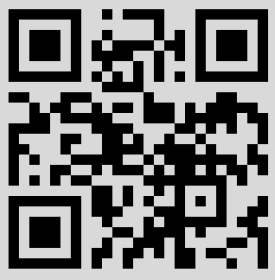




\title{
Асимптотика решений при больших временах для нелинейных уравнений типа Соболева
}

\author{
Е. И. Кайкина, П. И. Наумкин, И. А. Шишмарёв
}

Исследовано асимптотическое поведение при больших временах решений задачи Коши для нелинейного уравнения типа Соболева с диссипацией. Используемый нами подход в случае малых начальных данных основан на детальном изучении функции Грина линейной задачи и применении метода сжимающих отображений. Значительное внимание уделено также случаю больших начальных данных. В суперкритическом случае асимптотика имеет квазилинейный характер. Асимптотическое поведение решений нелинейного уравнения типа Соболева с критической нелинейностью неконвективного типа отличается от поведения решений соответствующего линейного уравнения логарифмической поправкой. Для критической конвективной нелинейности, а также для субкритической неконвективной нелинейности доказано, что главный член асимптотики решения при больших временах дается автомодельным решением. Для уравнений Соболева с конвективной нелинейностью асимптотика решений в субкритическом случае представима в виде произведения волны разряжения и ударной волны.

Библиография: 84 названия.

Ключевые слова: нелинейное уравнение типа Соболева с диссипацией, задача Коши, асимптотическое поведение решения при больших временах, критический показатель нелинейности.

\section{СоДЕРЖАНИЕ}

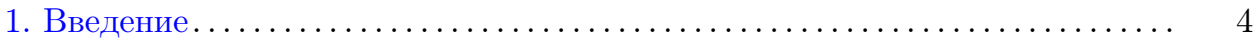

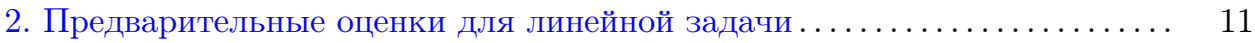

3. Асимптотически слабая нелинейность ........................ 17

3.1. Малые начальные данные .......................... 17

3.2. Большие начальные данные . . . . . . . . . . . . . . . . . . . . . . 25

4. Критическая неконвективная нелинейность ................. 33

4.1. Малые начальные данные ........................... 33

4.2. Большие начальные данные............................. 40

Работа выполнена при поддержке РФФИ (грант № 08-01-00-200-а).

(С) Е. И. КАЙКИНА, П. И. НАУмКиН, И. А. ШиШмАРЁв, 2009 
5. Критическая конвективная нелинейность .................. 41

5.1. Малые начальные данные . . . . . . . . . . . . . . . . . . . . . . . . 41

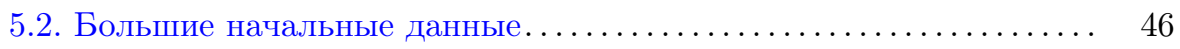

6. Субкритическая неконвективная нелинейность ................. 48

6.1. Малые начальные данные . . . . . . . . . . . . . . . . . . . . . . . 48

6.2. Большие начальные данные ......................... 57

7. Субкритическая конвективная нелинейность ................. 58

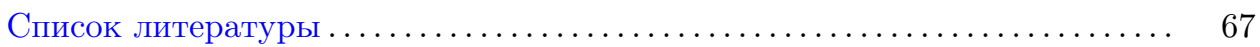

\section{1. Введение}

В настоящей работе мы даем обзор известных результатов и развиваем единый систематический подход к изучению асимптотики при больших временах решений задачи Коши для уравнений типа Соболева

$$
\begin{gathered}
\partial_{t}\left(u+(-\Delta)^{k} u\right)-\alpha \Delta u=\mathscr{N}(u), \quad x \in \mathbb{R}^{n}, \quad t>0, \\
u(0, x)=u_{0}(x), \quad x \in \mathbb{R}^{n},
\end{gathered}
$$

где $\alpha>0, k=1,2$, с различными нелинейными членами $\mathscr{N}=\lambda|u|^{\sigma} u, \mathscr{N}=$ $\lambda \operatorname{div}\left(|\nabla u|^{\sigma} \nabla u\right), \lambda \in \mathbb{R}$, и $\mathscr{N}=(\lambda \cdot \nabla)|u|^{\sigma} u, \lambda \in \mathbb{R}^{n}, \sigma>0$.

Уравнения типа Соболева описывают различные физические процессы, и их исследованию посвящено большое количество работ, так что математическая теория этих уравнений занимает важное место в современной математической физике. В работе С. Л. Соболева [1] было выведено линейное уравнение $\partial_{t}^{2} \Delta u+\alpha^{2} \partial_{x_{3}}^{2} u=0, x \in \mathbb{R}^{3}$, описывающее малые колебания во вращающейся жидкости, и впервые была математически строго рассмотрена задача, отличная от задач типа Коши-Ковалевской. Эта работа вызвала большой интерес к исследованию неклассических уравнений - так называемых уравнений типа Соболева. Впоследствии исследования С.Л. Соболева были продолжены в [2]-[10] и др.

Никоим образом не претендуя на полноту, дадим все же некоторый обзор литературы, посвященной исследованию уравнений типа Соболева. В работе [11] получено линейное уравнение типа Соболева $\partial_{t}(\Delta u+\beta u)+\Delta u=0$, где $\beta \neq 0$, описывающее нестационарный процесс фильтрации жидкости в пористой среде с трещинами. В работах [3], [12] доказана глобальная во времени разрешимость начально-краевых задач для уравнений в стратифицированных жидкостях. В работе [13] построено фундаментальное решение оператора внутренних гравитационных волн $\partial_{t}^{2}\left(\Delta u-\beta^{2} u\right)+\omega_{0}^{2}\left(\partial_{x_{1}}^{2}+\partial_{x_{2}}^{2}\right) u=0, x \in \mathbb{R}^{3}$, где $\beta$ - параметр стратификации, $\omega_{0}$ - частота Вейселя-Брента. Дисперсионные уравнения третьего и пятого порядка исследовались в работах [14]-[29], где, в частности, изучались вопросы глобального во времени существования и разрушения решений.

Приведем обзор результатов, посвященных исследованию диссипативных нелинейных уравнений типа Соболева. По поводу локальной разрешимости в классах $\mathbf{C}$ и $\mathbf{L}^{2}$ для уравнений типа Соболева в операторно-дифференциальной форме отметим книгу [30], где также рассмотрен вопрос обоснования методов конечномерной аппроксимации и метода Галёркина. В книге [31] установлены 
условия разрешимости задач в весовых соболевских пространствах, доказаны теоремы единственности и получены априорные $\mathbf{L}^{p}$-оценки решений задачи Коши и смешанных задач для дифференциальных уравнений и систем, не разрешенных относительно старшей производной, а также изучены асимптотические свойства решений некоторых задач гидродинамики. Уравнения типа Соболева со знакопеременным или необратимым оператором при старшей производной по времени в абстрактной постановке $\mathbf{B} \frac{d}{d t} u+\mathbf{L} u=f$, где $\mathbf{L}$ и $\mathbf{B}$ - самосопряженные или диссипативные операторы в некотором гильбертовом пространстве, изучались в работе [32]. Применение полугрупп операторов с нетривиальными ядрами, а также ограниченных, секториальных и радиальных операторов к общей теории сингулярных уравнений типа Соболева получило развитие в [33]. Вырожденные уравнения типа Соболева исследовались с абстрактной точки зрения в книге [34], где сингулярные уравнения соболевского типа сведены к дифференциальному уравнению с многозначным линейным оператором. Уравнения типа Соболева с двумя нелинейностями рассматривались в работе [35], где развивается метод многозначных линейных операторов, предложенный в работе [34].

Иногда в литературе уравнения типа Соболева называются также псевдопараболическими уравнениями. В работе [36] исследован вопрос о единственности решения задачи Коши для квазилинейного уравнения псевдопараболического типа $\partial_{t}(u-c \Delta u)=\varphi(u)$ в классе растущих функций $\varphi(u)$. В работе [37] получены результаты типа теорем существования-несуществования, в частности, доказано разрушение за конечное время положительного решения нелинейного уравнения типа Соболева с источником $\partial_{t}(u-\Delta u)-\Delta \psi(u)+q(u)=0$. Оптимальное управление в линейных задачах для уравнений псевдопараболического типа исследовалось в работе [38]. В [39] доказано существование обобщенных решений для линейных уравнений соболевского типа более высокого порядка. Отметим также, что изучению задач для квазилинейных уравнений соболевского типа посвящены работы [40] и [41]. Исследованию псевдопараболических уравнений методами теории функций комплексного переменного посвящена работа [42]. Доказательству принципа максимума для уравнений псевдопараболического типа посвящена работа [43]. Псевдопараболические уравнения с монотонной нелинейностью изучались в работе [44], где в развернутом виде применялся классический метод монотонности в приложении к различным классам уравнений математической физики, в частности, к нелинейным уравнениям соболевского типа с монотонными нелинейностями.

Перейдем к обзору результатов о несуществовании и разрушении решений для уравнений соболевского типа. Прежде всего отметим классическую работу Х. Фуджиты [45] о несуществовании положительного решения для полулинейного уравнения параболического типа, где автор получил оптимальный результат о разрушении положительного решения задачи Коши для полулинейного параболического уравнения $u_{t}=\Delta u+u^{1+\sigma}, x \in \mathbb{R}^{n}$, пользуясь свойствами фундаментального решения оператора теплопроводности. Кроме того, были определены критические показатели нелинейности $\sigma_{\mathrm{cr}}=2 / n$ (так называемые показатели Фуджиты) такие, что при $\sigma>\sigma_{\text {cr }}$ всегда существует классическое

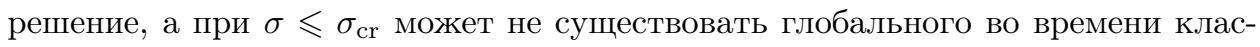


сического решения (по крайней мере положительного). Метод доказательства несуществования решения для некоторых классов краевых задач, основанный на использовании принципа максимума, развит в работах [46], [47]. В монографии [48] развит оригинальный метод исследования вопросов разрушения решений дифференциальных неравенств. Благодаря обобщению понятия емкости авторам [48] удается доказать разрушение решений для любого типа дифференциальных неравенств. Надеемся, что этот подход применим также к случаю дифференциальных уравнений соболевского типа. В классической работе [49] получены достаточные условия разрушения решений для классов квазилинейных уравнений параболического типа с помощью признаков сравнения, построения верхних и нижних решений, а также методом неограниченных коэффициентов Фурье. Эта же методика может быть применена и при исследовании вопросов разрушения для псевдопараболических уравнений.

В монографии [10] дано всестороннее освещение современного состояния теории линейных и нелинейных уравнений соболевского типа. Дан вывод модельных линейных уравнений соболевского типа высокого порядка, применимых в теории плазмы и для описания квазистационарных процессов в сплошных электромагнитных средах. Получены достаточные, близкие к необходимым, условия разрушения за конечное время и глобальной разрешимости. Обсуждаются методы численного решения уравнения типа Соболева.

В работе [50] была предложена новая задача, для которой удалось получить оптимальный результат Фуджиты. В работе [51] для исследования класса квазилинейных параболических уравнений был применен принцип максимума. В классической работе Г. Левина [52] энергетическим методом доказано разрушение сильного и слабого обобщенных решений задачи Коши для операторно-дифференциального уравнения вида $A \frac{d u}{d t}+L u=F(u)$ при достаточно больших начальных данных $u(0)=u_{0}$, где линейные операторы $A$ и $L-$ положительно определенные и самосопряженные, а нелинейность $F(u)$ имеет симметричную производную Фреше. Отметим, что в работах [53], [14] энергетический метод Левина развит в следующих направлениях: рассмотрен случай нелинейных операторов $A$ и $L$ и получены двусторонние оценки времени разрушения, далее в случае линейного оператора $A$ найдены оптимальные двусторонние оценки не только для времени, но и для скорости разрушения, наконец, рассмотрен случай волновых уравнений псевдопараболического типа.

В случае глобальной во времени разрешимости исследовались вопросы асимптотического поведения решений рассматриваемых задач при больших временах, теория рассеяния и устойчивость решений типа уединенных волн как для одномерных, так и для многомерных уравнений типа Бенджамена-Бона-Махони-Бюргерса и Розенау-Бюргерса. Асимптотическое поведение при больших временах решения задачи Коши для уравнения типа Соболева с нелинейностью конвективного типа изучалось в работах [54]-[63], [29]. Асимптотическое поведение при больших временах решений задачи Коши для нелинейного уравнения типа Соболева с неконвективной нелинейностью степенного вида (1) изучалось в работах [64]-[67].

Как правило, уравнения типа Соболева не удается решить явно, поэтому различные аналитические методы их исследования играют важную роль. Од- 
ним из наиболее эффективных способов качественного анализа нелинейных дифференциальных уравнений в частных производных являются асимптотические методы, позволяющие получать явные представления решений. Асимптотические формулы дают возможность выявить такие свойства решений, как скорость убывания или роста в различных областях, монотонный или осциллирующий характер их поведения, зависимость от начальных возмущений с течением времени и т. д. Также интересно исследовать влияние нелинейных членов уравнений типа Соболева на асимптотику их решений. Так, например, в отличие от соответствующих линейных уравнений решения нелинейных задач могут быстро осциллировать, они могут расти или убывать быстрее, чем решения соответствующих линейных уравнений, приближаться к автомодельному решению и т. п. Заметим, что очень трудно выявить эту информацию с помощью вычислительного эксперимента, так что асимптотические методы важны не только с теоретической точки зрения, но также широко используются на практике в дополнение к численным методам.

Асимптотические методы для нелинейных эволюционных уравнений являются относительно молодой областью математики, и общая теория еще далека от своего завершения. Описание асимптотики при больших временах решений нелинейных эволюционных уравнений требует принципиально новых подходов. Например, требования бесконечной дифференцируемости решения и компактности его носителя, обычно допустимые для линейных уравнений, являются слишком ограничительными в нелинейной теории. Асимптотические методы сложны даже в случае линейных эволюционных уравнений, поскольку требуют не только глобального во времени существования решений, но также ряда дополнительных априорных оценок (часто в весовых нормах) для того, чтобы можно было оценить разность между решением и его асимптотическим приближением. Обобщенные решения обычно непригодны для асимптотической теории, поэтому мы рассматриваем классические или полуклассические решения в пространствах Лебега или Соболева. Каждый тип нелинейности приходится рассматривать отдельно, в особенности при рассмотрении не малых начальных данных.

Опишем кратко основные утверждения работы. Мы делим нелинейные члены в уравнении типа Соболева (1) на три класса: асимптотически слабые, критические и сильные (или субкритические). Кроме того, критические и субкритические нелинейности подразделяются на конвективные и неконвективные. Асимптотика при больших временах решений задачи Коши для уравнения типа Соболева (1) оказывается различной во всех этих случаях. С эвристической точки зрения роль критичности показателя нелинейности нетрудно понять, если сравнить скорости убывания линейных и нелинейных членов уравнения на фундаментальном решении, так что критический показатель является критерием равновесия асимптотик линейных и нелинейных частей на фундаментальном решении соответствующего линейного оператора Соболева. Именно поэтому возникает асимптотическая подчиненность нелинейного оператора при $\sigma>\sigma_{\text {сr }}$ и в силу этого сохраняется в главном асимптотика фундаментального решения и для соответствующей нелинейной задачи Коши. Похожая ситуация имеет место для показателя Фуджиты, который отграничивает об- 
ласть глобального во времени существования решений от области разрушения решений за конечное время. Следует, однако, подчеркнуть, что критический показатель с точки зрения асимптотики решений для различных нелинейных уравнений, вообще говоря, может отличаться от показателя Фуджиты.

В суперкритическом случае нелинейность является асимптотически слабой и не влияет существенно на асимптотическую форму решений. Так, для уравнения Соболева (1) при $k=1$ со степенной нелинейностью $\mathscr{N}=\lambda|u|^{\sigma} u, \lambda \in \mathbb{R}$, в суперкритическом случае $\sigma>2 / n$ доказано существование единственного решения задачи Коши с достаточно малыми начальными возмущениями и для этого решения вычислен главный член асимптотики: $u \approx A t^{-n / 2} e^{-|x|^{2} /(4 \alpha t)}$ при больших временах $t \rightarrow \infty$ равномерно по $x \in \mathbb{R}^{n}$, где $A$ - некоторая постоянная (см. теорему 3 ниже). Отметим, что с точностью до константы эта же асимптотика имеет место и для линейного уравнения Соболева. В теореме 4 мы покажем, что нелинейность $\mathscr{N}=(\lambda \cdot \nabla)|u|^{\sigma} u, \lambda \in \mathbb{R}^{n}$, в уравнении Соболева (1) с $k=2$ является слабой при $\sigma>1 / n$, а именно, для достаточно малых начальных возмущений будет доказано существование единственного решения задачи Коши и вычислена его асимптотика при $t \rightarrow \infty$, равномерная по $x \in \mathbb{R}^{n}$. Эта же асимптотика справедлива и для решения задачи Коши для уравнения Соболева (1) с $k=2$ и нелинейностью $\mathscr{N}=\lambda \operatorname{div}\left(|\nabla u|^{\sigma} \nabla u\right), \lambda \in \mathbb{R}$, при всех $\sigma>0$ (см. теорему 5 ниже). Поскольку нелинейность зачастую приводит к разрушению решений за конечное время, нам приходится рассматривать малые начальные данные, чтобы иметь возможность изучать глобальные во времени решения. Однако в ряде случаев нам удается получить асимптотику при больших временах также и для произвольных (т. е. не малых) начальных условий. Так, в теоремах 6,7 и 8 соответственно для уравнения Соболева (1) при $k=1$ со степенной нелинейностью $\mathscr{N}=\lambda|u|^{\sigma} u, \lambda<0, \sigma>2 / n$, для уравнения Соболева (1) при $k=2$ с нелинейностью $\mathscr{N}=(\lambda \cdot \nabla)|u|^{\sigma} u, \lambda \in \mathbb{R}^{n}, \sigma \geqslant 1, n=1,2$, и для уравнения Соболева (1) при $k=2$ с нелинейностью $\mathscr{N}=\lambda \operatorname{div}\left(|\nabla u|^{\sigma} \nabla u\right)$, $\lambda>0, \sigma>0, n=1$, мы избавимся от требования малости начальных данных.

В критическом случае нелинейность неконвективного типа определяет логарифмическую поправку в главном члене асимптотики по сравнению с соответствующим линейным уравнением. Так, для уравнения Соболева (1) при $k=1$ со степенной нелинейностью $\mathscr{N}=\lambda|u|^{\sigma} u, \lambda \in \mathbb{R}$, в критическом случае $\sigma=2 / n$ доказано существование единственного решения задачи Коши с достаточно малыми начальными возмущениями такими, что общая масса $\theta=\int_{\mathbb{R}^{n}} u_{0}(x) d x$ удовлетворяет условию $\lambda \theta<0$, и для этого решения получена асимптотика $u \approx A(\log t)^{-n / 2} t^{-n / 2} e^{-|x|^{2} /(4 \alpha t)}$ (см. теорему 10). Если же $\lambda<0$, то мы можем отказаться от требования малости начальных возмущений, однако при этом асимптотика в критическом случае $\sigma=2 / n$ имеет альтернативный характер, а именно, либо решение имеет главный член асимптотики $u \approx A(\log t)^{-n / 2} t^{-n / 2} e^{-|x|^{2} /(4 \alpha t)}$, либо оно убывает более быстро во времени: $u=O\left(t^{-n / 2} \log ^{-n / 2-1} t\right)$ (см. теорему 12$)$.

Для критической конвективной нелинейности доказывается, что главный член асимптотики при больших временах дается автомодельным решением. Так, для уравнения Соболева (1) при $k=2$ с критической конвективной нели- 
нейностью $\mathscr{N}=(\lambda \cdot \nabla)|u|^{\sigma} u, \lambda \in \mathbb{R}^{n}, \sigma=1 / n, n=1,2,3$, в теореме 14 доказано, что при малых начальных возмущениях с ненулевой общей массой, $\theta \neq 0$, существует единственное решение задачи Коши и для этого решения справедлива асимптотика $u \approx t^{-n / 2} V\left(x t^{-1 / 2}\right)$, где $V(\xi)$ является решением интегрального уравнения

$$
V(\xi)=\theta \widetilde{G}_{0}(\xi)-\int_{0}^{1} \frac{d z}{z(1-z)^{n / 2}} \int_{\mathbb{R}^{n}} \widetilde{G}_{0}\left(\frac{\xi-y \sqrt{z}}{\sqrt{1-z}}\right) \mathscr{N}(V(y)) d y .
$$

Здесь $\widetilde{G}_{0}(\xi)=(4 \pi \alpha)^{-n / 2} e^{-|\xi|^{2} /(4 \alpha)}$. А в случае не малых начальных данных $u_{0}$, имеющих ненулевую общую массу, $\theta \neq 0$, в теореме 14 доказано, что существует единственное решение задачи Коши для уравнения Соболева (1) при $k=2$ с критической конвективной нелинейностью $\mathscr{N}=(\lambda \cdot \nabla)|u|^{\sigma} u, \sigma=1$, $\lambda \in \mathbb{R}, n=1$. Более того, для этого решения справедлива асимптотика $u(t) \approx t^{-1 / 2} f_{\theta}\left(x t^{-1 / 2}\right)$, где функция

$$
f_{\theta}(\chi)=-2 \sqrt{\mu} \frac{\partial}{\partial \chi} \log \left(\cosh \frac{\theta}{4}-\sinh \left(\frac{\theta}{4}\right) \operatorname{Erf}\left(\frac{\chi}{2 \sqrt{\mu}}\right)\right)
$$

является автомодельным решением уравнения Бюргерса $u_{t}+u u_{x}-\mu u_{x x}=0$, определяемым общей массой $\theta$ начальных данных, здесь $\operatorname{Erf}(x)-$ интеграл ошибок.

В случае субкритической неконвективной нелинейности главный член асимптотики решения при больших временах также дается автомодельным решением. В теореме 19 рассматривается уравнение типа Соболева (1) при $k=1$ со степенной нелинейностью $\mathscr{N}=\lambda|u|^{\sigma} u, \lambda \in \mathbb{R}$, в субкритическом случае $0<\sigma<2 / n$. В предположении, что начальные возмущения $u_{0}$ достаточно малы и $\lambda \theta<0$, а также что величина $\sigma$ достаточно близка к значению $2 / n$, мы докажем, что задача Коши имеет единственное глобальное во времени решение. Более того, для этого решения справедлива асимптотика $u \approx A t^{-1 / \sigma} V\left(x t^{-1 / 2}\right)$, где $A$ - некоторая постоянная, а функция $V(\xi)$ является решением интегрального уравнения типа (2). Если $\lambda<0, n=1,2$, то мы можем освободиться от условия малости начальных данных и установить (см. теорему 20) глобальное во времени существование решений задачи Коши с начальными данными $u_{0}$, имеющими ненулевую общую массу $\theta \neq 0$. Это решение имеет ту же асимптотику при $t \rightarrow \infty$, что и в теореме 19 .

Наконец, для уравнения типа Соболева (1) при $k=1$ с конвективной нелинейностью $\mathscr{N}=(\lambda \cdot \nabla)|u|^{\sigma} u, \lambda \in \mathbb{R}, n=1$, в субкритическом случае $\sigma \in(0,1)$ мы представим асимптотику решений в виде произведения волны разряжения и ударной волны (см. теорему 23).

Отметим, что подобное асимптотическое поведение решений было ранее обнаружено для нелинейного уравнения теплопроводности с неконвективной нелинейностью $u_{t}-\Delta u+u^{1+\sigma}=0$ (см. работу [68] по поводу суперкритического случая $\sigma>2 / n$, работу [69] в критическом случае $\sigma=2 / n$ и работы [70]-[73] в субкритическом случае $\sigma \in(0,2 / n))$, а также для нелинейного уравнения теплопроводности с конвективной нелинейностью $u_{t}-\Delta u+(\lambda \cdot \nabla)|u|^{\sigma} u=0$, где $\sigma>0$ (см. [74], [75]). 
Будем обозначать всюду

$$
\langle x\rangle=\sqrt{1+|x|^{2}}, \quad\{x\}=\frac{|x|}{\langle x\rangle}, \quad|x|=\left(\sum_{j=1}^{n} x_{j}^{2}\right)^{1 / 2},
$$

так что имеет место представление $|x|=\{x\}\langle x\rangle$ для всех $x \in \mathbb{R}^{n}$. Обозначим через $[\alpha]$ целую часть числа $\alpha \in \mathbb{R} ;$ более подробно, $[\alpha]=\max _{k \leqslant \alpha} k$, где $k \in \mathbb{Z}$. Через $\mathscr{F} \phi$ или $\hat{\phi}$ будем обозначать прямое преобразование Фурье функции $\phi$, т. e.

$$
\hat{\phi}(\xi)=(2 \pi)^{-\frac{n}{2}} \int_{\mathbb{R}^{n}} e^{-i x \xi} \phi(x) d x
$$

тогда обратное преобразование Фурье дается формулой

$$
\mathscr{F}^{-1} \phi=(2 \pi)^{-\frac{n}{2}} \int_{\mathbb{R}^{n}} e^{i x \xi} \phi(x) d x,
$$

где через $x \xi$, как обычно, обозначаем $\xi x=(\xi \cdot x)$ скалярное произведение в $\mathbb{R}^{n}$. Пространство Лебега обозначаем, как обычно, через $\mathbf{L}^{p}\left(\mathbb{R}^{n}\right)=\left\{\varphi \in \mathscr{S}^{\prime}\right.$ : $\left.\|\varphi\|_{\mathbf{L}^{p}}<\infty\right\}$, с нормой

$$
\|\varphi\|_{\mathbf{L}^{p}}=\left(\int_{\mathbb{R}^{n}}|\varphi(x)|^{p} d x\right)^{\frac{1}{p}} \text { при } 1 \leqslant p<\infty
$$

и $\|\varphi\|_{\mathbf{L}^{\infty}}=\operatorname{ess.sup}_{x \in \mathbb{R}^{n}}|\varphi(x)|$ при $p=\infty$. Определим также весовое пространство Лебега $\mathbf{L}^{p, a}$

$$
\mathbf{L}^{p, a}=\left\{\phi \in \mathbf{L}^{p}:\|\phi\|_{\mathbf{L}^{p, a}}<\infty\right\}
$$

с нормой $\|\phi\|_{\mathbf{L}^{p, a}} \equiv\left\|\langle x\rangle^{a} \phi(x)\right\|_{\mathbf{L}^{p}}$. Пространство Соболева определим следующим образом: $\mathbf{W}_{p}^{k}\left(\mathbb{R}^{n}\right)=\left\{\varphi \in \mathbf{L}^{p}:\left\|\left\langle i \partial_{x}\right\rangle^{k} \varphi(x)\right\|_{\mathbf{L}^{p}}<\infty\right\}$ при $1 \leqslant p \leqslant \infty$ и целом $k$. Также обозначим $\mathbf{H}^{k}\left(\mathbb{R}^{n}\right)=\mathbf{W}_{2}^{k}\left(\mathbb{R}^{n}\right)$, где $k \geqslant 0$ (см. [76]). Весовое пространство Соболева определим как $\mathbf{W}_{p}^{s, a}\left(\mathbb{R}^{n}\right)=\left\{\varphi \in \mathbf{L}^{p}:\left\|\left\langle i \partial_{x}\right\rangle^{s} \varphi(x)\right\|_{\mathbf{L}^{p, a}}<\infty\right\}$ для любых $s, a \in \mathbb{R}, 1 \leqslant p \leqslant \infty$. В частности, при $p=2$ обозначим также $\mathbf{H}^{s, a}\left(\mathbb{R}^{n}\right)=\mathbf{W}_{2}^{s, a}\left(\mathbb{R}^{n}\right)$. Очевидно равенство $\mathbf{W}_{p}^{s, 0}\left(\mathbb{R}^{n}\right)=\mathbf{W}_{p}^{s}\left(\mathbb{R}^{n}\right)$. Норму обычного пространства Соболева $\mathbf{H}^{k}=\mathbf{H}^{k, 0}$ будем задавать также следующим образом: $\|\phi\|_{\mathbf{H}^{k}}^{2}=\sum_{j=0}^{k}\left\|\partial^{j} \phi\right\|_{\mathbf{L}^{2}}^{2}$. Мы будем также рассматривать $\mathbf{C}(\mathbf{I} ; \mathbf{B})-$ пространство непрерывных функций, отображающих интервал времени I в банахово пространство В. Различные постоянные будем обозначать одной и той же буквой $C$. Введем дробную производную $\partial_{x_{j}}^{\beta}$ для всех $\beta \geqslant 0$ и $j=1, \ldots, n$ следующим образом (см. [77]):

$$
\partial_{x_{j}}^{\beta} \phi=\mathscr{F}^{-1}\left(\left(i \xi_{j}\right)^{\varrho-1}\left(i \xi_{j}\right)^{k+1} \hat{\phi}\right),
$$

где $k=[\beta], \varrho=\beta-k \in(0,1)$ и $\left(i \xi_{j}\right)^{\varrho-1}$ обозначает главное значение аналитической функции $\left(i \xi_{j}\right)^{\varrho-1}=\left|\xi_{j}\right|^{\varrho-1} \exp \left\{i(\varrho-1) \arg \left(i \xi_{j}\right)\right\}$. Поскольку

$$
\mathscr{F}^{-1}\left(\left(i \xi_{j}\right)^{\varrho-1}\right)=\frac{\sqrt{\pi} y^{-\varrho}}{\sqrt{2} \Gamma(1-\varrho) \cos \left(\frac{\pi}{2}(\varrho-1)\right)}
$$


при $y>0, \varrho \in(0,1)$, где $Г$ - гамма-функция Эйлера, то интегрирование по частям дает следующее представление:

$$
\partial_{x_{j}}^{\beta} \phi(x)=\frac{2 \pi}{\Gamma(1-\varrho)} \int_{0}^{\infty}\left(\partial_{x_{j}}^{k} \phi(\breve{x}(y))-\partial_{x_{j}}^{k} \phi(x)\right) y^{-1-\varrho} d y,
$$

где $x=\left(x_{1}, \ldots, x_{n}\right)$, а $\breve{x}(y)=\left(x_{1}, \ldots, x_{j}+y, \ldots, x_{n}\right)$.

В следующем разделе мы получим предварительные оценки для оператора Грина линейной задачи Коши для уравнений типа Соболева. В последующих разделах мы изучим асимптотическое поведение при больших временах решений задачи Коши для нелинейного уравнения типа Соболева (1) в суперкритическом, критическом и субкритическом случаях.

\section{2. Предварительные оценки для линейной задачи}

Рассмотрим линейную задачу Коши

$$
\begin{gathered}
\partial_{t}\left(u+(-\Delta)^{k} u\right)-\alpha \Delta u=f, \quad x \in \mathbb{R}^{n}, \quad t>0, \\
u(0, x)=u_{0}(x), \quad x \in \mathbb{R}^{n},
\end{gathered}
$$

где $\alpha>0, k=1,2$. С помощью принципа Дюамеля запишем решение задачи (3) в виде

$$
u(t)=\mathscr{G}_{k}(t) u_{0}+\int_{0}^{t} \mathscr{G}_{k}(t-\tau) \mathscr{B}_{k} f(\tau) d \tau,
$$

где оператор Грина $\mathscr{G}_{k}(t)$ задается через обратное преобразование Фурье следующим образом:

$$
\mathscr{G}_{k}(t) \phi=\mathscr{F}^{-1}\left(\exp \left\{-\frac{\alpha t|\xi|^{2}}{1+|\xi|^{2 k}}\right\} \hat{\phi}\right),
$$

a оператор $\mathscr{B}_{k} \phi=\int_{\mathbb{R}^{n}} B_{k}(x-y) \phi(y) d y$ задан своим ядром

$$
B_{k}(x)=(2 \pi)^{-\frac{n}{2}} \int_{\mathbb{R}^{n}} e^{i \xi x}\left(1+|\xi|^{2 k}\right)^{-1} d \xi .
$$

Отметим, что справедлива оценка $\left|B_{k}(x)\right| \leqslant C|x|^{1-n} e^{-|x|}$ (см. [78], [79]), так что имеем

$$
\begin{aligned}
\left\|\mathscr{B}_{k} \phi\right\|_{\mathbf{L}^{p, a}} & =\left\|\int_{\mathbb{R}^{n}} B_{k}(x-y) \phi(y) d y\right\|_{\mathbf{L}^{p, a}} \\
& \leqslant\left\|B_{k}\right\|_{\mathbf{L}^{1, a}}\|\phi\|_{\mathbf{L}^{p, a}} \leqslant C\|\phi\|_{\mathbf{L}^{p, a}} \int_{\mathbb{R}^{n}}|x|^{a+1-n} e^{-|x|} d x \leqslant C\|\phi\|_{\mathbf{L}^{p, a}}
\end{aligned}
$$

для всех $1 \leqslant p \leqslant \infty$ и $a \geqslant 0$.

Рассмотрим оценки для оператора $\mathscr{G}_{1}(t)$ в нормах весовых пространств Лебега. Обозначим через $\widetilde{G}_{0}(x)=(4 \pi \alpha)^{-n / 2} e^{-|x|^{2} /(4 \alpha)}$ ядро функции Грина для уравнения теплопроводности. 
ЛЕмма 1. Справедливы оченки

u

$$
\begin{gathered}
\left\||x|^{b} \partial^{\beta} \mathscr{G}_{1}(t) \phi\right\|_{\mathbf{L}^{p}} \leqslant \\
C\langle t\rangle^{-\frac{n}{2}\left(\frac{1}{r}-\frac{1}{p}\right)+\frac{b}{2}-\frac{|\beta|}{2}}\left(\|\phi\|_{\mathbf{L}^{r}}+\|\phi\|_{\left.\mathbf{W}_{p}^{|\beta|, b}\right)}\right. \\
+\langle t\rangle^{-\frac{n}{2}\left(\frac{1}{r}-\frac{1}{p}\right)-\frac{|\beta|}{2}}\|\phi\|_{\mathbf{L}^{r, b}}
\end{gathered}
$$

$$
\begin{aligned}
\left\||x|^{b}\left(\mathscr{G}_{1}(t) \phi-\theta\langle t\rangle^{-\frac{n}{2}} \widetilde{G}_{0}\left(x\langle t\rangle^{-\frac{1}{2}}\right)\right)\right\|_{\mathbf{L}^{p}} \\
\quad \leqslant C\langle t\rangle^{-\frac{n}{2}\left(1-\frac{1}{p}\right)+\frac{b-a}{2}}\left(\left\||x|^{a} \phi\right\|_{\mathbf{L}^{1}}+\left\||x|^{b} \phi\right\|_{\mathbf{L}^{p}}\right)
\end{aligned}
$$

для всех $t>0$, где $1 \leqslant r \leqslant p \leqslant \infty, 0 \leqslant|\beta| \leqslant n+1,0 \leqslant b \leqslant a \leqslant 1, \theta=$ $\int_{\mathbb{R}^{n}} \phi(x) d x$

ДокАЗАТЕЛьство. Представим оператор Грина $\mathscr{G}_{1}(t)$ в виде

$$
\mathscr{G}_{1}(t) \phi=\mathscr{G}_{0}(t) \phi+e^{-\alpha t} \sum_{k=0}^{n} \frac{\alpha^{k} t^{k}}{k !} \mathscr{B}_{1}^{k} \phi+\mathscr{R}_{1}(t) \phi,
$$

где

$$
\mathscr{G}_{0}(t) \phi=\langle t\rangle^{-\frac{n}{2}} \int_{\mathbb{R}^{n}} \widetilde{G}_{0}\left((x-y)\langle t\rangle^{-\frac{1}{2}}\right) \phi(y) d y,
$$

а остаток $\mathscr{R}_{1}(t) \phi=\int_{\mathbb{R}^{n}} R_{1}(t, x-y) \phi(y) d y$ имеет ядро $R_{1}(t, x)=\mathscr{F}^{-1} \widehat{R}_{1}(t, \xi)$, где

$$
\widehat{R}_{1}(t, \xi)=e^{-\alpha t|\xi|^{2}\langle\xi\rangle^{-2}}-e^{-\alpha\langle t\rangle|\xi|^{2}}-e^{-\alpha t} \sum_{k=0}^{n} \frac{\alpha^{k} t^{k}}{k !}\langle\xi\rangle^{-2 k} .
$$

Благодаря оценкам леммы 1.28 из книги [67] найдем

$$
\begin{gathered}
\left\||x|^{b} \partial^{\beta} \mathscr{G}_{0}(t) \phi\right\|_{\mathbf{L}^{p}} \leqslant \\
C\langle t\rangle^{-\frac{n}{2}\left(\frac{1}{r}-\frac{1}{p}\right)+\frac{b-|\beta|}{2}}\left(\|\phi\|_{\mathbf{L}^{r}}+\|\phi\|_{\mathbf{W}_{p}^{|\beta|}}\right) \\
+C\langle t\rangle^{-\frac{n}{2}\left(\frac{1}{r}-\frac{1}{p}\right)-\frac{|\beta|}{2}}\|\phi\|_{\mathbf{L}^{r, b}}
\end{gathered}
$$

и

$$
\left\||x|^{b}\left(\mathscr{G}_{0}(t) \phi-\theta\langle t\rangle^{-\frac{n}{2}} \widetilde{G}_{0}\left(x\langle t\rangle^{-\frac{1}{2}}\right)\right)\right\|_{\mathbf{L}^{p}} \leqslant C\langle t\rangle^{-\frac{n}{2}\left(1-\frac{1}{p}\right)+\frac{b-a}{2}}\left\||x|^{a} \phi\right\|_{\mathbf{L}^{1}}
$$

для всех $t>0$. Кроме того, при $t>0$ и $1 \leqslant p \leqslant \infty$ выполнено неравенство

$$
\left\||x|^{b} \partial^{\beta} e^{-\alpha t} \sum_{k=0}^{n} \frac{\alpha^{k} t^{k}}{k !} \mathscr{B}_{1}^{k} \phi\right\|_{\mathbf{L}^{p}} \leqslant C\langle t\rangle^{n} e^{-\alpha t}\|\phi\|_{\mathbf{W}_{p}^{|\beta|, b}}
$$

Оценим теперь остаток $\mathscr{R}_{1}(t) \phi$. При всех $\xi \in \mathbb{R}^{n}, t>0,0 \leqslant j \leqslant n+2$ имеет место неравенство

$$
\left|\partial_{\xi_{l}}^{j} \widehat{R}_{1}(t, \xi)\right| \leqslant C\langle t\rangle^{\frac{j}{2}-1} e^{-\frac{\alpha}{2} t|\xi|^{2}}+C\langle t\rangle^{n+1} e^{-\alpha t}\langle\xi\rangle^{-2 n-2},
$$

из которого выводится следующая оценка для ядра $R_{1}(t, x):$ при $0 \leqslant|\beta| \leqslant n+1$

$$
\begin{aligned}
\left|\partial^{\beta} R_{1}(t, x)\right| \leqslant C & \left\||\xi|^{|\beta|} \widehat{R}_{1}(t, \xi)\right\|_{\mathbf{L}^{1}} \leqslant C\langle t\rangle^{-1}\left\||\xi|^{|\beta|} e^{-\frac{\alpha}{2} t|\xi|^{2}}\right\|_{\mathbf{L}^{1}} \\
& +C\langle t\rangle^{n+1} e^{-\alpha t}\left\||\xi|^{\beta}\langle\xi\rangle^{-2 n-2}\right\|_{\mathbf{L}^{1}} \leqslant C\langle t\rangle^{-1} t^{-\frac{n}{2}-\frac{|\beta|}{2}} .
\end{aligned}
$$


С другой стороны, интегрируя по частям $n+2$ раза по переменной $\xi_{l}$, получаем

$$
\begin{aligned}
& \left|\partial^{\beta} R_{1}(t, x)\right| \leqslant C|x|^{-n-2} \sum_{l=1}^{n}\left|\partial^{\beta} \int_{\mathbb{R}^{n}} e^{i \xi x} \partial_{\xi_{l}}^{n+2} \widehat{R}_{1}(t, \xi) d \xi\right| \\
& \quad \leqslant C|x|^{-n-2} t^{\frac{n+2}{2}}\langle t\rangle^{-1}|||\xi|^{|\beta|} e^{-\frac{\alpha}{2} t|\xi|^{2}}\left\|_{\mathbf{L}^{1}}+C|x|^{-n-2} e^{-\frac{\alpha}{2} t}\right\||\xi|^{|\beta|}\langle\xi\rangle^{-2 n-2} \|_{\mathbf{L}^{1}} \\
& \quad \leqslant C\left(|x| t^{-\frac{1}{2}}\right)^{-n-2}\langle t\rangle^{-1} t^{-\frac{n}{2}-\frac{|\beta|}{2}}+C|x|^{-n-2} e^{-\frac{\alpha}{2} t} .
\end{aligned}
$$

Комбинируя эти две оценки, имеем для всех $x \in \mathbb{R}^{n}, t>0$

$$
\left|\partial^{\beta} R_{1}(t, x)\right| \leqslant C\left\langle|x| t^{-\frac{1}{2}}\right\rangle^{-n-2}\langle t\rangle^{-1} t^{-\frac{n}{2}-\frac{|\beta|}{2}}+C\langle x\rangle^{-n-2} e^{-\frac{\alpha}{2} t} .
$$
Ввиду (8), применяя неравенство Юнга для сверток с $\frac{1}{p}=\frac{1}{q}+\frac{1}{r}-1$, находим
при $t \in(0,1]$

$$
\begin{aligned}
\left\|\partial^{\beta} \mathscr{R}_{1}(t) \phi\right\|_{\mathbf{L}^{p}} & \leqslant C\left\|\int_{\mathbb{R}^{n}} R_{1}(t, x-y) \partial^{\beta} \phi(y) d y\right\|_{\mathbf{L}^{p}} \\
& \leqslant C\left\|R_{1}(t)\right\|_{\mathbf{L}^{1}}\|\phi\|_{\mathbf{W}_{p}^{|\beta|}} \leqslant C\|\phi\|_{\mathbf{W}_{p}^{|\beta|}}
\end{aligned}
$$

и при $t>1$

$$
\begin{aligned}
\left\|\partial^{\beta} \mathscr{R}_{1}(t) \phi\right\|_{\mathbf{L}^{p}} & \leqslant C\left\|\int_{\mathbb{R}^{n}} \partial^{\beta} R_{1}(t, x-y) \phi(y) d y\right\|_{\mathbf{L}^{p}} \leqslant C\left\|\partial^{\beta} R_{1}(t)\right\|_{\mathbf{L}^{q}}\|\phi\|_{\mathbf{L}^{r}} \\
& \leqslant C t^{-\frac{n}{2}-1-\frac{|\beta|}{2}}\left\|\left\langle|x| t^{-\frac{1}{2}}\right\rangle^{-n-2}\right\|_{\mathbf{L}^{q}}\|\phi\|_{\mathbf{L}^{r}}+C e^{-\frac{\alpha}{2} t}\left\|\langle x\rangle^{-n-2}\right\|\left\|_{\mathbf{L}^{q}}\right\| \phi \|_{\mathbf{L}^{r}} \\
& \leqslant C t^{-\frac{n}{2}\left(\frac{1}{r}-\frac{1}{p}\right)-1-\frac{|\beta|}{2}}\|\phi\|_{\mathbf{L}^{r}},
\end{aligned}
$$

где $1 \leqslant r \leqslant p \leqslant \infty$. Из (5)-(9) следуют оценки леммы. Лемма 1 доказана.

Теперь оценим в весовых лебеговских нормах оператор $\mathscr{G}_{2}(t)$.

Лемма 2. При всех $t>0$ справедливы оценки

$$
\begin{gathered}
\left\||x|^{b} \partial^{\beta} \mathscr{G}_{2}(t) \phi\right\|_{\mathbf{L}^{p}} \leqslant C\langle t\rangle^{-\frac{n}{2}\left(\frac{1}{r}-\frac{1}{p}\right)-\frac{|\beta|}{2}}\|\phi\|_{\mathbf{L}^{r, b}}+C\langle t\rangle^{-\frac{n}{2}\left(\frac{1}{r}-\frac{1}{p}\right)+\frac{b-|\beta|}{2}}\|\phi\|_{\mathbf{L}^{r}} \\
+C\langle t\rangle^{-\frac{m-|\beta-\alpha|}{2}}\|\phi\|_{\mathbf{W}_{p}^{|\alpha|+m, b}}
\end{gathered}
$$

где $1 \leqslant r \leqslant p \leqslant \infty, 0 \leqslant \alpha \leqslant \beta,|\beta-\alpha|<m, 0 \leqslant b<1, u$

$$
\begin{gathered}
\left\||x|^{b}\left(\mathscr{G}_{2}(t) \phi-\theta\langle t\rangle^{-\frac{n}{2}} \widetilde{G}_{0}\left(x\langle t\rangle^{-\frac{1}{2}}\right)\right)\right\|_{\mathbf{L}^{p}} \leqslant C\langle t\rangle^{-\frac{n}{2}\left(1-\frac{1}{p}\right)+\frac{b-a}{2}}\left\||x|^{a} \phi\right\|_{\mathbf{L}^{1}} \\
+C\langle t\rangle^{-\frac{m}{2}}\|\phi\|_{\mathbf{W}_{p}^{m, b}}
\end{gathered}
$$

где $1 \leqslant p \leqslant \infty, m>0,0 \leqslant b \leqslant a<1, \theta=\int_{\mathbb{R}^{n}} \phi(x) d x$.

ДокАЗАтЕльство. Представим оператор Грина $\mathscr{G}_{2}(t)$ следующим образом:

$$
\mathscr{G}_{2}(t) \phi=\mathscr{F}^{-1} \exp \left\{-\frac{\alpha t|\xi|^{2}}{1+|\xi|^{4}}\right\} \hat{\phi}(\xi)=\int_{\mathbb{R}^{n}} G_{2}(t, x-y)\langle i \nabla\rangle^{m} \phi(y) d y, \quad m>0,
$$


где ядро $G_{2}(t, x)$ имеет вид

$$
G_{2}(t, x)=(2 \pi)^{-\frac{n}{2}} \mathscr{F}^{-1}\left(\exp \left\{-\frac{\alpha t|\xi|^{2}}{1+|\xi|^{4}}\right\}\langle\xi\rangle^{-m}\right) .
$$

Рассмотрим сначала случай $0<t \leqslant 1$. С помощью интегрирования по частям находим, что

$$
\begin{aligned}
\left|\partial^{\beta-\alpha} G_{2}(t, x)\right| & \leqslant C|x|^{-l} \sum_{j=1}^{n}\left|\int_{\mathbb{R}^{n}} e^{i \xi x} \xi^{\beta-\alpha} \partial_{\xi_{j}}^{l}\left(\exp \left\{-\frac{\alpha t|\xi|^{2}}{1+|\xi|^{4}}\right\}\langle\xi\rangle^{-m}\right) d \xi\right| \\
& \leqslant C|x|^{-l}\left|\int_{\mathbb{R}^{n}}\langle\xi\rangle^{|\beta-\alpha|-m-l} d \xi\right| \leqslant C|x|^{-l}
\end{aligned}
$$

для всех $x \in \mathbb{R}^{n} \backslash\{0\}, 0<t \leqslant 1$ при $l>n+|\beta-\alpha|-m$. Комбинируя это неравенство с соотношениями $l=n+\gamma+|\beta-\alpha|-m$ и $l=n+\gamma+|\beta-\alpha|+b$, $\gamma \in(0, m-|\beta-\alpha|)$, получаем

$$
\left|\partial^{\beta-\alpha} G_{2}(t, x)\right| \leqslant C|x|^{-n-\gamma-|\beta-\alpha|+m}\langle x\rangle^{|\beta-\alpha|-m-b}
$$

для всех $x \in \mathbb{R}^{n} \backslash\{0\}, 0<t \leqslant 1$. Значит, справедлива оценка

$$
\left\|\partial^{\beta-\alpha} G_{2}(t)\right\|_{\mathbf{L}^{1}}+\left\||x|^{b} \partial^{\beta-\alpha} G_{2}(t)\right\|_{\mathbf{L}^{1}} \leqslant C
$$

при $0 \leqslant|\beta-\alpha|<m$. Тогда по неравенству Юнга приходим к первой оценке леммы:

$$
\begin{aligned}
&\left\||x|^{b} \partial^{\beta} \mathscr{G}_{2}(t) \phi\right\|_{\mathbf{L}^{p}} \leqslant\left\|\int_{\mathbb{R}^{n}}|x-y|^{b} \partial^{\beta-\alpha} G_{2}(t, x-y) \partial^{\alpha}\langle i \nabla\rangle^{m} \phi(y) d y\right\|_{\mathbf{L}^{p}} \\
&+\left\|\int_{\mathbb{R}^{n}} \partial^{\beta-\alpha} G_{2}(t, x-y)\langle y\rangle^{b} \partial^{\alpha}\langle i \nabla\rangle^{m} \phi(y) d y\right\|_{\mathbf{L}^{p}} \leqslant C\|\phi\|_{\mathbf{W}_{p}^{|\alpha|+m, b}}
\end{aligned}
$$

для всех $0<t \leqslant 1$, при $0 \leqslant|\alpha| \leqslant|\beta|, 0 \leqslant|\beta-\alpha|<m$.

Теперь рассмотрим $t \geqslant 1$. Введем срезку $\varphi_{1} \in \mathbf{C}_{0}^{\infty}\left(\mathbb{R}^{n}\right)$ такую, что $\varphi_{1}(\xi)=1$ при $|\xi| \leqslant 1$ и $\varphi_{1}(\xi)=0$ при $|\xi| \geqslant 2$, положим также $\varphi_{2}(\xi)=1-\varphi_{1}(\xi)$ и обозначим

$$
K_{j}(t, x)=(2 \pi)^{-\frac{n}{2}} \mathscr{F}^{-1}\left(\exp \left\{-\frac{\alpha t|\xi|^{2}}{1+|\xi|^{4}}\right\}\langle\xi\rangle^{-m} \varphi_{j}(\xi)\right), \quad j=1,2,
$$
так что $G_{2}(t, x)=K_{1}(t, x)+K_{2}(t, x)$. Отметим, что функция $\exp \left\{-\frac{\alpha t|\xi|^{2}}{1+|\xi|^{4}}\right\} \times$
$\langle\xi\rangle^{-m} \varphi_{1}(\xi) \in \mathbf{C}^{N}\left(\mathbb{R}^{n} \backslash\{0\}\right), N=n+2$, удовлетворяет оценке

$$
\left|\partial_{\xi_{j}}^{l}\left(\exp \left\{-\frac{\alpha t|\xi|^{2}}{1+|\xi|^{4}}\right\}\langle\xi\rangle^{-m} \varphi_{1}(\xi)\right)\right| \leqslant C t^{\frac{l}{2}} \exp \left\{-\frac{\alpha}{3} t|\xi|^{2}\right\}
$$

для всех $t \geqslant 1, \xi \in \mathbb{R}^{n} \backslash\{0\}, l=0,1, \ldots, n+2, j=1, \ldots, n$. Благодаря (10) ядро $K_{1}(t, x)$ можно оценить следующим образом:

$$
\begin{aligned}
\left|\partial^{\beta} K_{1}(t, x)\right| & \leqslant C\left\|\exp \left\{-\frac{\alpha t|\xi|^{2}}{1+|\xi|^{4}}\right\}\langle\xi\rangle^{-m} \xi^{\beta} \varphi_{1}(\xi)\right\|_{\mathbf{L}^{1}} \\
& \leqslant C\left\||\xi|^{\beta} \exp \left\{-\frac{\alpha}{3} t|\xi|^{2}\right\}\right\|_{\mathbf{L}^{1}} \leqslant C t^{-\frac{n+|\beta|}{2}} .
\end{aligned}
$$


С другой стороны, с помощью интегрирования $n+2$ раза по частям по $\xi_{l}$ получаем неравенство

$$
\begin{aligned}
\left|\partial^{\beta} K_{1}(t, x)\right| & \leqslant C|x|^{-n-2} \sum_{j=1}^{n}\left|\int_{\mathbb{R}^{n}} e^{i \xi x} \xi^{\beta} \partial_{\xi_{j}}^{n+2}\left(\exp \left\{-\frac{\alpha t|\xi|^{2}}{1+|\xi|^{4}}\right\}\langle\xi\rangle^{-m} \varphi_{1}(\xi)\right) d \xi\right| \\
& \leqslant C|x|^{-n-2} t^{\frac{n+2}{2}}\left\||\xi|^{\beta} \exp \left\{-\frac{\alpha}{3} t|\xi|^{2}\right\}\right\|_{\mathbf{L}^{1}} \leqslant C\left(|x| t^{-\frac{1}{2}}\right)^{-n-2} t^{-\frac{n+|\beta|}{2}} .
\end{aligned}
$$

Комбинируя последние два неравенства, имеем для всех $x \in \mathbb{R}^{n}, t \geqslant 1$

$$
\left|\partial^{\beta} K_{1}(t, x)\right| \leqslant C\left\langle|x| t^{-\frac{1}{2}}\right\rangle^{-n-2} t^{-\frac{n+|\beta|}{2}}
$$

при любых $|\beta| \geqslant 0$. Ввиду (11), для $\mathscr{K}_{1}(t) \phi=\int_{\mathbb{R}^{n}}\langle i \nabla\rangle^{m} K_{1}(t, x-y) \phi(y) d y$ с помощью неравенства Юнга с $\frac{1}{p}=\frac{1}{q}+\frac{1}{r}-1$ получаем

$$
\begin{aligned}
& \left\||x|^{b} \partial^{\beta} \mathscr{K}_{1}(t) \phi\right\|_{\mathbf{L}^{p}} \leqslant C\left\|\int_{\mathbb{R}^{n}} \partial^{\beta}\langle i \nabla\rangle^{m} K_{1}(t, x-y)\langle y\rangle^{b} \phi(y) d y\right\|_{\mathbf{L}^{p}} \\
& \quad+C\left\|\int_{\mathbb{R}^{n}}|x-y|^{b} \partial^{\beta}\langle i \nabla\rangle^{m} K_{1}(t, x-y) \phi(y) d y\right\|_{\mathbf{L}^{p}} \\
& \leqslant C\left\|K_{1}(t)\right\|_{\mathbf{L}^{q}}\|\phi\|_{\mathbf{L}^{r, b}}+C\left\|K_{1}(t)\right\|_{\mathbf{L}^{q, b}}\|\phi\|_{\mathbf{L}^{r}} \\
& \leqslant C t^{-\frac{n+|\beta|}{2}}\left\|\left\langle|x| t^{-\frac{1}{2}}\right\rangle^{-n-2}\right\|_{\mathbf{L}^{q}}\|\phi\|_{\mathbf{L}^{r, b}}+C t^{-\frac{n+|\beta|}{2}}\left\|\left\langle|x| t^{-\frac{1}{2}}\right\rangle^{-n-2}\right\|_{\mathbf{L}^{q, b}}\|\phi\|_{\mathbf{L}^{r}} \\
& \leqslant C t^{-\frac{n}{2}\left(\frac{1}{r}-\frac{1}{p}\right)-\frac{|\beta|}{2}}\|\phi\|_{\mathbf{L}^{r, b}}+C t^{-\frac{n}{2}\left(\frac{1}{r}-\frac{1}{p}\right)+\frac{b-|\beta|}{2}}\|\phi\|_{\mathbf{L}^{r}}
\end{aligned}
$$

для всех $t>0$, где $1 \leqslant r \leqslant p \leqslant \infty, 0 \leqslant b<1$. Поскольку для всех $j>n$

$$
\left.\left|\int_{|\xi|>1} \exp \left\{-\frac{\alpha t}{2|\xi|^{4}}\right\}\right| \xi\right|^{-j} d \xi \mid \leqslant C \int_{1}^{\infty} \exp \left\{-\frac{\alpha t}{2 y^{2}}\right\} y^{n-1-j} d y \leqslant C\langle t\rangle^{-\frac{j-n}{2}}
$$

то интегрирование по частям дает при $l>n+|\beta-\alpha|-m$

$$
\begin{aligned}
\left|\partial^{\beta-\alpha} K_{2}(t, x)\right| & \leqslant C|x|^{-l} \sum_{j=1}^{n}\left|\int_{\mathbb{R}^{n}} e^{i \xi x} \partial_{\xi_{j}}^{l}\left(\exp \left\{-\frac{\alpha t|\xi|^{2}}{1+|\xi|^{4}}\right\}\langle\xi\rangle^{-m} \xi^{\beta-\alpha} \varphi_{2}(\xi)\right) d \xi\right| \\
& \leqslant\left. C|x|^{-l}\left|\int_{|\xi|>1} \exp \left\{-\frac{\alpha t}{2|\xi|^{4}}\right\}\right| \xi\right|^{|\beta-\alpha|-m-l} d \xi \mid \\
& \leqslant C|x|^{-l} t^{-\frac{l+m-|\beta-\alpha|-n}{2}}
\end{aligned}
$$

для всех $x \in \mathbb{R}^{n} \backslash\{0\}, t \geqslant 1$. Комбинируя это неравенство с соотношениями $l=n+\gamma+|\beta-\alpha|-m$ и $l=n+\gamma+|\beta-\alpha|+1, \gamma \in(0, m-|\beta-\alpha|)$, находим

$$
\left|\partial^{\beta-\alpha} K_{2}(t, x)\right| \leqslant C|x|^{-n-\gamma-|\beta-\alpha|+m} t^{-\frac{\gamma}{2}}\langle|x| \sqrt{t}\rangle^{-m-1}
$$

для всех $x \in \mathbb{R}^{n} \backslash\{0\}, t \geqslant 1$. Следовательно,

$$
\left\||x|^{b} \partial^{\beta-\alpha} K_{2}(t)\right\|_{\mathbf{L}^{1}} \leqslant C t^{\frac{|\beta-\alpha|}{2}-\frac{m+b}{2}}
$$


для всех $t \geqslant 1$. Поэтому применение неравенства Юнга к оператору $\mathscr{K}_{2}(t) \phi=$ $\int_{\mathbb{R}^{n}} K_{2}(t, x-y)\langle i \nabla\rangle^{m} \phi(y) d y$ дает оценку

$$
\begin{aligned}
& \left\|\partial^{\beta} \mathscr{K}_{2}(t) \phi\right\|_{\mathbf{L}^{p, b}} \leqslant C\left\|\int_{\mathbb{R}^{n}}|x-y|^{b} \partial^{\beta-\alpha} K_{2}(t, x-y)\langle i \nabla\rangle^{m} \phi(y) d y\right\|_{\mathbf{L}^{p}} \\
& \quad+C\left\|\int_{\mathbb{R}^{n}} \partial^{\beta-\alpha} K_{2}(t, x-y)\langle y\rangle^{b}\langle i \nabla\rangle^{m} \phi(y) d y\right\|_{\mathbf{L}^{p}} \leqslant C t^{\frac{|\beta-\alpha|}{2}-\frac{m}{2}}\|\phi\|_{\mathbf{W}_{p}^{|\alpha|+m, b}}
\end{aligned}
$$

для всех $t \geqslant 1$, где $0 \leqslant|\beta-\alpha|<m$. Таким образом, первая оценка леммы имеет место.

Для доказательства второй оценки леммы запишем $\mathscr{G}_{2}(t) \phi=\mathscr{G}_{0}(t) \phi+\mathscr{R}_{2}(t) \phi$, где остаток $\mathscr{R}_{2}(t) \phi$ имеет вид

$$
\mathscr{R}_{2}(t) \phi=\int_{\mathbb{R}^{n}} R_{2}(t, x-y)\langle i \nabla\rangle^{m} \phi(y) d y
$$

с ядром $R_{2}(t, x)=\widetilde{K}_{1}(t, x)+\widetilde{K}_{2}(t, x)$, где

$$
\widetilde{K}_{j}(t, x)=(2 \pi)^{-\frac{n}{2}} \mathscr{F}^{-1}\left(\left(\exp \left\{-\frac{\alpha t|\xi|^{2}}{1+|\xi|^{4}}\right\}-\exp \left\{-\alpha\langle t\rangle|\xi|^{2}\right\}\right)\langle\xi\rangle^{-m} \varphi_{j}(\xi)\right) .
$$

Как и выше, получаем оценку $\left|\langle i \nabla\rangle^{m} \widetilde{K}_{1}(t, x)\right| \leqslant C\left\langle|x|\langle t\rangle^{-1 / 2}\right\rangle^{-n-2}\langle t\rangle^{-n / 2-1}$ для всех $x \in \mathbb{R}^{n}, t>0$, благодаря которой для оператора $\widetilde{\mathscr{K}}_{1}(t) \phi=\int_{\mathbb{R}^{n}}\langle i \nabla\rangle^{m} \times$ $\widetilde{K}_{1}(t, x-y) \phi(y) d y$ с помощью неравенства Юнга при $\frac{1}{p}=\frac{1}{q}+\frac{1}{r}-1$ выводим оценку

$$
\begin{aligned}
\left\||x|^{b} \widetilde{\mathscr{K}}_{1}(t) \phi\right\|_{\mathbf{L}^{p}} \leqslant & C\left\|\int_{\mathbb{R}^{n}}\langle i \nabla\rangle^{m} \widetilde{K}_{1}(t, x-y)\langle y\rangle^{b} \phi(y) d y\right\|_{\mathbf{L}^{p}} \\
& +C\left\|\int_{\mathbb{R}^{n}}|x-y|^{b}\langle i \nabla\rangle^{m} \widetilde{K}_{1}(t, x-y) \phi(y) d y\right\|_{\mathbf{L}^{p}} \\
\leqslant & C\langle t\rangle^{-1-\frac{n}{2}\left(\frac{1}{r}-\frac{1}{p}\right)}\|\phi\|_{\mathbf{L}^{r, b}}+C\langle t\rangle^{-1-\frac{n}{2}\left(\frac{1}{r}-\frac{1}{p}\right)+\frac{b}{2}}\|\phi\|_{\mathbf{L}^{r}}
\end{aligned}
$$

для всех $t>0$, где $1 \leqslant r \leqslant p \leqslant \infty, 0 \leqslant b<1$. Аналогично, имеем

$$
\left\||x|^{b} \widetilde{K}_{2}(t)\right\|_{\mathbf{L}^{1}} \leqslant C t^{-\frac{m+b}{2}}
$$

для всех $t>0$. Поэтому для $\widetilde{\mathscr{K}}_{2}(t) \phi=\int_{\mathbb{R}^{n}} \widetilde{K}_{2}(t, x-y)\langle i \nabla\rangle^{m} \phi(y) d y$ неравенство Юнга дает

$$
\begin{aligned}
\left\|\mathscr{K}_{2}(t) \phi\right\|_{\mathbf{L}^{p, b}} \leqslant C \| & \int_{\mathbb{R}^{n}}|x-y|^{b} \widetilde{K}_{2}(t, x-y)\langle i \nabla\rangle^{m} \phi(y) d y \|_{\mathbf{L}^{p}} \\
& +C\left\|\int_{\mathbb{R}^{n}} \widetilde{K}_{2}(t, x-y)\langle y\rangle^{b}\langle i \nabla\rangle^{m} \phi(y) d y\right\|_{\mathbf{L}^{p}} \leqslant C t^{-\frac{m}{2}}\|\phi\|_{\mathbf{W}_{p}^{m, b}}
\end{aligned}
$$

для всех $t>0$. Так что в силу (6) справедлива вторая оценка леммы. Лемма 2 доказана. 


\section{3. Асимптотически слабая нелинейность}

3.1. Малые начальные данные. В этом разделе мы развиваем общий подход к изучению асимптотики при больших временах для решений эволюционных диссипативных уравнений с асимптотически слабой нелинейностью. Рассмотрим задачу Коши

$$
\begin{gathered}
u_{t}+\mathscr{N}(u)+\mathscr{L} u=0, \quad x \in \mathbb{R}^{n}, \quad t>0, \\
u(0, x)=u_{0}(x), \quad x \in \mathbb{R}^{n},
\end{gathered}
$$

где $\mathscr{L}$ - это линейный псевдодифференциальный оператор, $\mathscr{L} \phi=\mathscr{F}^{-1} L(\xi) \hat{\phi}(\xi)$ с $L(\xi)=\frac{\alpha|\xi|^{2}}{1+|\xi|^{2 k}}, k=1,2, \alpha>0$, а $\mathscr{N}(u)$ - нелинейный член такой, что $\mathscr{N}(0)=0$. С помощью принципа Дюамеля перепишем задачу Коши (12) в виде интегрального уравнения

$$
\begin{aligned}
u(t) & =\mathscr{G}(t) u_{0}-\int_{0}^{t} \mathscr{G}(t-\tau) \mathscr{N}(u(\tau)) d \tau \\
& =\int_{\mathbb{R}^{n}} G(t, x-y) u_{0}(y) d y-\int_{0}^{t} \int_{\mathbb{R}^{n}} G(t-\tau, x-y) \mathscr{N}(u)(\tau, y) d y d \tau,
\end{aligned}
$$

где $\mathscr{G}$ - оператор Грина соответствующей линейной задачи,

$$
\mathscr{G}(t) \phi=\overline{\mathscr{F}}_{\xi \rightarrow x} e^{-t L(\xi)} \hat{\phi}(\xi)=\int_{\mathbb{R}^{n}} G(t, x-y) \phi(y) d y .
$$

Под решением задачи Коши (12) будем понимать решение соответствующего интегрального уравнения (13).

Пусть $\mathbf{Z}$ - некоторое метрическое пространство функций на $\mathbb{R}^{n}$, кроме того, зафиксируем некоторое полное метрическое пространство $\mathbf{X}$ функций на $[0, \infty) \times \mathbb{R}^{n}$. Докажем сначала глобальное во времени существование решений задачи Коши (12).

Теорема 1. Пусть начальные возмущения $u_{0} \in \mathbf{Z}$ достаточно маль. Предположим, что определен оператор Грина $\mathscr{G}: \mathbf{Z} \rightarrow \mathbf{X}$, удовлетворяющий оченке

$$
\|\mathscr{G} \phi\|_{\mathbf{x}} \leqslant C\left\|_{\phi}\right\|_{\mathbf{z}}
$$

Предположим также, что $\int_{0}^{t} \mathscr{G}(t-\tau) \mathscr{N}(v(\tau)) d \tau \in \mathbf{X}$ для любой функиии $v \in \mathbf{X}$ и справедлива ощенка

$$
\left\|\int_{0}^{t} \mathscr{G}(t-\tau)(\mathscr{N}(w(\tau))-\mathscr{N}(v(\tau))) d \tau\right\|_{\mathbf{X}} \leqslant C\|w-v\|_{\mathbf{X}}\left(\|w\|_{\mathbf{X}}+\|v\|_{\mathbf{X}}\right)^{\sigma}
$$

для любых $v, w \in \mathbf{X}$, где $\sigma>0$. Тогда существует единственное решение $u \in \mathbf{X}$ задачи Коши (12), удовлетворяющее неравенству

$$
\|u\|_{\mathbf{x}} \leqslant C\left\|u_{0}\right\|_{\mathbf{z}}
$$


ДоКАЗАТЕЛЬСТво. Применим принцип сжимающих отображений в шаре $\mathbf{X}_{\rho}=\left\{\phi \in \mathbf{X}:\|\phi\|_{\mathbf{X}} \leqslant \rho\right\}$ с радиусом $\rho=(2 C)^{-1}\left\|u_{0}\right\|_{\mathbf{Z}}>0$, где постоянная $C>0$ взята из условий (14), (15). Для всех $v \in \mathbf{X}_{\rho}$ определим отображение $\mathscr{M}(v)$ формулой

$$
\mathscr{M}(v)=\mathscr{G}(t) u_{0}-\int_{0}^{t} \mathscr{G}(t-\tau) \mathscr{N}(v(\tau)) d \tau .
$$

Докажем сначала неравенство $\|\mathscr{M}(v)\|_{\mathbf{X}} \leqslant \rho$, где $\rho>0$ достаточно мало. Из условий теоремы и интегральной формулы (17) получим

$$
\begin{aligned}
\|\mathscr{M}(v)\|_{\mathbf{X}} & \leqslant\left\|\mathscr{G} u_{0}\right\|_{\mathbf{X}}+\left\|\int_{0}^{t} \mathscr{G}(t-\tau) \mathscr{N}(v(\tau)) d \tau\right\|_{\mathbf{X}} \\
& \leqslant C\left\|u_{0}\right\|_{\mathbf{z}}+C\|v\|_{\mathbf{X}}^{\sigma+1} \leqslant \frac{\rho}{2}+C \rho^{\sigma+1}<\rho,
\end{aligned}
$$

поскольку $\rho>0$ достаточно мало. Следовательно, отображение $\mathscr{M}$ преобразует шар $\mathbf{X}_{\rho}$ в себя. Подобным образом получим оценку $\|\mathscr{M}(w)-\mathscr{M}(v)\|_{\mathbf{X}} \leqslant$ $(1 / 2)\|w-v\|_{\mathbf{X}}$, откуда видно, что $\mathscr{M}$ является сжимающим отображением. Таким образом, существует единственное решение $u \in \mathbf{X}$ задачи Коши (12), удовлетворяющее неравенству (16). Теорема 1 доказана.

Будем называть функцию $G_{0} \in \mathbf{X}$ асимптотическим ядром оператора Грина $\mathscr{G}$ в пространствах $\mathbf{X}$ и $\mathbf{Z}$, если для некоторого непрерывного функционала $f: \mathbf{Z} \rightarrow \mathbb{R}$ выполняется оценка

$$
\left\|\langle t\rangle^{\gamma}\left(\mathscr{G}(t) \phi-G_{0}(t) f(\phi)\right)\right\|_{\mathbf{X}} \leqslant C\|\phi\|_{\mathbf{Z}}
$$

для всех $\phi \in \mathbf{Z}$, где $\gamma>0$. Ниже функционал $f(\phi)$ будем выбирать в виде $f(\phi)=\int_{\mathbb{R}^{n}} \phi(x) d x=(2 \pi)^{n / 2} \hat{\phi}(0)$, также функционал $f$ может иметь форму моментов. Будем называть нелинейность $\mathscr{N}(u)$ в уравнении (12) асимптотически слабой в пространстве $\mathbf{X}$, если сходится интеграл $\int_{0}^{\infty} f(\mathscr{N}(u(\tau))) d \tau$ для любой функции $u \in \mathbf{X}$.

В следующей теореме мы установим достаточные условия для нахождения асимптотики решений задачи Коши (12) с асимптотически слабой нелинейностью.

Теорема 2. Пусть $u_{0} \in \mathbf{Z}$. Предположим, ито существует асимптотическое ядро $G_{0}$ для оператора Грина $\mathscr{G}$ в пространствах $\mathbf{X}, \mathbf{Z}$. Пусть нелинейность $\mathscr{N}(u)$ в уравнении (12) является асимптотически слабой в пространстве $\mathbf{X}$. Предположим, ито существует единственное глобальное решение $u \in \mathbf{X}$ задачи Коши (12), удовлетворяющее оченкам

$$
\begin{gathered}
\left\|\langle t\rangle^{\gamma} \int_{0}^{\frac{t}{2}}\left(\mathscr{G}(t-\tau) \mathscr{N}(u(\tau))-G_{0}(t-\tau) f(\mathscr{N}(u(\tau)))\right) d \tau\right\|_{\mathbf{X}} \\
+\left\|\langle t\rangle^{\gamma} \int_{\frac{t}{2}}^{t} \mathscr{G}(t-\tau) \mathscr{N}(u(\tau)) d \tau\right\|_{\mathbf{X}} \leqslant C\|u\|_{\mathbf{X}}^{\sigma}
\end{gathered}
$$




$$
\begin{aligned}
\|\langle t\rangle^{\gamma} G_{0}(t) & \int_{\frac{t}{2}}^{\infty} f(\mathscr{N}(u(\tau))) d \tau \|_{\mathbf{X}} \\
& +\left\|\langle t\rangle^{\gamma} \int_{0}^{\frac{t}{2}}\left(G_{0}(t-\tau)-G_{0}(t)\right) f(\mathscr{N}(u(\tau))) d \tau\right\|_{\mathbf{X}} \leqslant C\|u\|_{\mathbf{X}}^{\sigma}
\end{aligned}
$$

где $\sigma>0, \gamma>0$. Тогда справедливо асимптотическое представление

$$
u(t)=A G_{0}(t)+\langle t\rangle^{-\gamma} R(t),
$$

где постоянная $A$ вычисляется по формуле

$$
A=f\left(u_{0}\right)-\int_{0}^{\infty} f(\mathscr{N}(u(\tau))) d \tau
$$

и остаток $R$ оченивается следующим образом: $\|R\|_{\mathbf{X}} \leqslant C\left\|u_{0}\right\|_{\mathbf{Z}}+C\|u\|_{\mathbf{X}}^{\sigma}$.

ЗАмечАниЕ 1. Мы можем гарантировать, что коэффициент $A$ в асимптотическом представлении (21) отличен от нуля, например, если $f\left(u_{0}\right) \neq 0$ и $\mathscr{N}(u)$ достаточно мало, что имеет место в случае малых решений, а также в случае уравнений конвективного типа, когда $f(\mathscr{N}(u)) \equiv 0$. Если же $A=0$ (например, для уравнений конвективного типа в случае, когда начальные возмущения имеют нулевое среднее значение $f\left(u_{0}\right)=0$ ), то формула $(21)$ дает лишь оценку убывания решения при больших временах.

ДокАЗАТЕЛьство. Из интегрального уравнения (13) получаем

$$
\begin{aligned}
\|\langle t\rangle^{\gamma}(u(t) & \left.-A G_{0}(t)\right)\left\|_{\mathbf{X}} \leqslant\right\|\langle t\rangle^{\gamma}\left(\mathscr{G}(t) u_{0}-G_{0}(t) f\left(u_{0}\right)\right) \|_{\mathbf{X}} \\
& +\left\|\langle t\rangle^{\gamma} \int_{0}^{\frac{t}{2}}\left(\mathscr{G}(t-\tau) \mathscr{N}(u(\tau))-G_{0}(t-\tau) \vartheta(\tau)\right) d \tau\right\|_{\mathbf{X}} \\
& +\left\|\langle t\rangle^{\gamma} \int_{\frac{t}{2}}^{t} \mathscr{G}(t-\tau) \mathscr{N}(u(\tau)) d \tau\right\|_{\mathbf{X}}+\left\|\langle t\rangle^{\gamma} G_{0}(t) \int_{\frac{t}{2}}^{\infty} \vartheta(\tau) d \tau\right\|_{\mathbf{X}} \\
& +\left\|\langle t\rangle^{\gamma} \int_{0}^{\frac{t}{2}}\left(G_{0}(t-\tau)-G_{0}(t)\right) \vartheta(\tau) d \tau\right\|_{\mathbf{X}},
\end{aligned}
$$

где $\vartheta(\tau)=f(\mathscr{N}(u(\tau)))$. В силу неравенств (18)-(20) правая часть (22) может быть оценена сверху величиной $C\left\|u_{0}\right\|_{\mathbf{z}}+C\|u\|_{\mathbf{X}}^{\sigma}$. Таким образом, из (22) вытекает асимптотика (21). Теорема 2 доказана.

Применим теоремы 1 и 2 к уравнению Соболева со степенной нелинейностью

$$
\begin{gathered}
\partial_{t}(u-\Delta u)-\alpha \Delta u=\lambda|u|^{\sigma} u, \quad x \in \mathbb{R}^{n}, \quad t>0, \\
u(0, x)=u_{0}(x), \quad x \in \mathbb{R}^{n},
\end{gathered}
$$

где $\alpha>0, \lambda \in \mathbb{R}$ (см. уравнение (1) с $k=1, \mathscr{N}=\lambda|u|^{\sigma} u, \sigma>0, \lambda \in \mathbb{R}$ ). Оказывается, что нелинейность в (23) является слабой при $\sigma>2 / n$. Уравнение $(23)$ можно рассматривать как многомерное обобщение уравнений Буссинеска; оно возникает в задачах фильтрации жидкостей в пористых средах [80], [37] и в 
теории кристаллических полупроводников [81]. Чтобы иметь возможность доказать существование глобальных во времени решений уравнения (23) с коэффициентом нелинейности $\lambda$ произвольного знака, приходится предполагать малость начальных возмущений, поскольку при $\lambda>0$ возможно разрушение решений за конечное время (см. [49], [48], [81]). В работе [64] доказан следующий результат.

Теорема 3. Пусть $\sigma>2 / n$. Предположим, что

$$
u_{0} \in \mathbf{L}^{1, a}\left(\mathbb{R}^{n}\right) \cap \mathbf{L}^{\infty}\left(\mathbb{R}^{n}\right), \quad a \in(0,1]
$$

причем норма $\left\|u_{0}\right\|_{\mathbf{L}^{1, a}}+\left\|u_{0}\right\|_{\mathbf{L}^{\infty}}$ достаточно мала. Тогда существует единственное решение $u \in \mathbf{C}\left([0, \infty) ; \mathbf{L}^{1, a}\left(\mathbb{R}^{n}\right) \cap \mathbf{L}^{\infty}\left(\mathbb{R}^{n}\right)\right)$ задачи Коши (23). Для этого решения при $t \rightarrow \infty$ равномерно по $x \in \mathbb{R}^{n}$ справедлива асимптотика $u(t, x)=A t^{-n / 2} e^{-|x|^{2} /(4 t)}+O\left(t^{-n / 2-\gamma}\right)$, где $A-$ некоторая постоянная, $0<\gamma<\min (a / 2,(n / 2) \sigma-1)$.

Теперь применим теоремы 1 и 2 к уравнению Соболева с конвективной нелинейностью

$$
\begin{gathered}
\partial_{t}\left(u+\Delta^{2} u\right)-\alpha \Delta u=(\lambda \cdot \nabla)|u|^{\sigma} u, \quad x \in \mathbb{R}^{n}, \quad t>0, \\
u(0, x)=u_{0}(x), \quad x \in \mathbb{R}^{n},
\end{gathered}
$$

где $\alpha>0, \sigma>0, \lambda \in \mathbb{R}^{n}$ (см. уравнение (1) с $k=2, \mathscr{N}=(\lambda \cdot \nabla)|u|^{\sigma} u, \sigma>0$, $\left.\lambda \in \mathbb{R}^{n}\right)$. В следующей теореме мы покажем, что нелинейность $\mathscr{N}=(\lambda \cdot \nabla)|u|^{\sigma} u$ в уравнении (24) является слабой при $\sigma>1 / n$.

Теорема 4. Пусть $\sigma>1 / n, n=1,2,3,4$. Предположим, что

$$
u_{0} \in \mathbf{L}^{1, a}\left(\mathbb{R}^{n}\right) \cap \mathbf{W}_{\infty}^{1}\left(\mathbb{R}^{n}\right), \quad a \in(0,1),
$$

причем норма $\left\|u_{0}\right\|_{\mathbf{L}^{1, a}}+\left\|u_{0}\right\|_{\mathbf{W}_{\infty}^{1}}$ достаточно мала. Тогда существует единственное решение $u \in \mathbf{C}\left([0, \infty) ; \mathbf{L}^{1, a}\left(\mathbb{R}^{n}\right) \cap \mathbf{W}_{\infty}^{1}\left(\mathbb{R}^{n}\right)\right)$ задачи Коши (24). Для этого решения при больших временах $t \rightarrow \infty$ равномерно по $x \in \mathbb{R}^{n}$ справедлива асимптотика $u(t, x)=\theta(4 \pi \alpha t)^{-n / 2} e^{-|x|^{2} /(4 \alpha t)}+O\left(t^{-n / 2-\gamma}\right)$, где $\theta=$ $\int_{\mathbb{R}^{n}} u_{0}(x) d x, 0<\gamma<\min (a / 2,(n / 2) \sigma-1)$.

ДокАЗАТЕЛЬство. Введем пространство

$$
\mathbf{X}=\left\{v \in \mathbf{C}\left([0, \infty) ; \mathbf{L}^{1, a}\left(\mathbb{R}^{n}\right) \cap \mathbf{W}_{\infty}^{1}\left(\mathbb{R}^{n}\right)\right):\|v\|_{\mathbf{X}}<\infty\right\}
$$

с нормой

$$
\|v\|_{\mathbf{X}}=\sup _{t>0}\left(\langle t\rangle^{-a / 2}\|v(t)\|_{\mathbf{L}^{1, a}}+\langle t\rangle^{n / 2}\|v(t)\|_{\mathbf{W}_{\infty}^{1}}\right) .
$$

Заметим, что $\sup _{t>0}\|\phi(t)\|_{\mathbf{L}^{1}} \leqslant\|\phi\|_{\mathbf{X}}$. Также обозначим

$$
\mathbf{Z}=\left\{\phi \in \mathbf{L}^{1, a}\left(\mathbb{R}^{n}\right) \cap \mathbf{W}_{\infty}^{1}\left(\mathbb{R}^{n}\right):\|\phi\|_{\mathbf{Z}}<\infty\right\}
$$

с нормой $\|\phi\|_{\mathbf{Z}}=\|\phi\|_{\mathbf{L}^{1, a}}+\|\phi\|_{\mathbf{W}_{\infty}^{1}}$. Определим отображение $\mathscr{M}(v)$ формулой (17), которая с учетом (4) принимает вид

$$
\mathscr{M}(v)=\mathscr{G}_{2}(t) u_{0}+\int_{0}^{t} \mathscr{B}_{2} \mathscr{G}_{2}(t-\tau) \mathscr{N}(v(\tau)) d \tau .
$$


Применение леммы 2 дает неравенства

$$
\left\|\mathscr{G}_{2} \phi\right\|_{\mathbf{W}_{\infty}^{1}} \leqslant C\langle t\rangle^{-2}\|\phi\|_{\mathbf{W}_{\infty}^{1}}+C\langle t\rangle^{-1 / 2}\|\phi\|_{\mathbf{L}^{1}}
$$

и

$$
\begin{gathered}
\left\|\int_{0}^{t} \mathscr{B}_{2} \mathscr{G}_{2}(t-\tau)(\lambda \cdot \nabla)\left(|w|^{\sigma} w(\tau)-|v|^{\sigma} v(\tau)\right) d \tau\right\|_{\mathbf{W}_{\infty}^{1}} \\
\leqslant C\left\||\nabla| \int_{0}^{t} \mathscr{B}_{2} \mathscr{G}_{2}(t-\tau)\left(|w|^{\sigma} w(\tau)-|v|^{\sigma} v(\tau)\right) d \tau\right\|_{\mathbf{L}^{\infty}} \\
\quad+C\left\||\nabla| \int_{0}^{t} \mathscr{B}_{2} \mathscr{G}_{2}(t-\tau)(\lambda \cdot \nabla)\left(|w|^{\sigma} w(\tau)-|v|^{\sigma} v(\tau)\right) d \tau\right\|_{\mathbf{L}^{\infty}} \\
\leqslant C \int_{0}^{\frac{t}{2}}\langle t-\tau\rangle^{-2}\left(\left\||w|^{\sigma} w(\tau)-|v|^{\sigma} v(\tau)\right\|_{\mathbf{W}_{\infty}^{1}}\right. \\
\left.\quad+\langle t-\tau\rangle^{-\frac{n+1}{2}}\left\||w|^{\sigma} w(\tau)-|v|^{\sigma} v(\tau)\right\|_{\mathbf{L}^{1}}\right) d \tau \\
\quad+C \int_{\frac{t}{2}}^{t}\langle t-\tau\rangle^{-\frac{1}{2}}\left|\left\||w|^{\sigma} w(\tau)-|v|^{\sigma} v(\tau)\right\|_{\mathbf{W}_{\infty}^{1}} d \tau .\right.
\end{gathered}
$$

Используя оценки

$$
\begin{gathered}
\left\||w|^{\sigma} w(\tau)-|v|^{\sigma} v(\tau)\right\|_{\mathbf{W}_{\infty}^{1}} \leqslant C\langle\tau\rangle^{-\frac{n}{2}(\sigma+1)}\|w-v\|_{\mathbf{X}}\left(\|w\|_{\mathbf{X}}^{\sigma}+\|v\|_{\mathbf{X}}^{\sigma}\right), \\
\left\||w|^{\sigma} w(\tau)-|v|^{\sigma} v(\tau)\right\|_{\mathbf{L}^{1}} \leqslant C\langle\tau\rangle^{-\frac{n}{2} \sigma}\|w-v\|_{\mathbf{X}}\left(\|w\|_{\mathbf{X}}^{\sigma}+\|v\|_{\mathbf{X}}^{\sigma}\right),
\end{gathered}
$$

получаем

$$
\begin{gathered}
\left\|\int_{0}^{t} \mathscr{B}_{2} \mathscr{G}_{2}(t-\tau)(\lambda \cdot \nabla)\left(|w|^{\sigma} w(\tau)-|v|^{\sigma} v(\tau)\right) d \tau\right\|_{\mathbf{W}_{\infty}^{1}} \\
\leqslant C\|w-v\|_{\mathbf{X}}\left(\|w\|_{\mathbf{X}}^{\sigma}+\|v\|_{\mathbf{X}}^{\sigma}\right)\left(\int_{\frac{t}{2}}^{t}\langle\tau\rangle^{-\frac{n}{2}(\sigma+1)}\langle t-\tau\rangle^{-\frac{1}{2}} d \tau\right. \\
\left.\quad+\int_{0}^{\frac{t}{2}}\left(\langle\tau\rangle^{-\frac{n}{2} \sigma}\langle t-\tau\rangle^{-\frac{n+1}{2}}+\langle\tau\rangle^{-\frac{n}{2}(\sigma+1)}\langle t-\tau\rangle^{-2}\right) d \tau\right) \\
\leqslant\langle t\rangle^{-\frac{n}{2}-\frac{\gamma}{2}}\|w-v\|_{\mathbf{X}}\left(\|w\|_{\mathbf{X}}^{\sigma}+\|v\|_{\mathbf{X}}^{\sigma}\right),
\end{gathered}
$$

поскольку $\sigma>1 / n, n=1,2,3,4$, где $0<\gamma<\min (1, n \sigma-1)$. Аналогично с помощью леммы 2 выводим неравенства

$$
\begin{gathered}
\left\|\mathscr{G}_{2} \phi\right\|_{\mathbf{L}^{1, a}} \leqslant C\langle t\rangle^{\frac{a}{2}}\|\phi\|_{\mathbf{L}^{1, a}}, \\
\left\|\mathscr{B}_{2} \mathscr{G}_{2}(t)(\lambda \cdot \nabla) \phi\right\|_{\mathbf{L}^{1, a}} \leqslant C\langle t\rangle^{-\frac{1}{2}}\|\phi\|_{\mathbf{L}^{1, a}}+C\langle t\rangle^{\frac{a-1}{2}}\|\phi\|_{\mathbf{L}^{1}},
\end{gathered}
$$

поэтому

$$
\begin{aligned}
\left\|\int_{0}^{t} \mathscr{B}_{2} \mathscr{G}_{2}(t-\tau)(\lambda \cdot \nabla)\left(|w|^{\sigma} w(\tau)-|v|^{\sigma} v(\tau)\right) d \tau\right\|_{\mathbf{L}^{1, a}} \\
\leqslant C \int_{0}^{t}\langle t-\tau\rangle^{-\frac{1}{2}}|||w|^{\sigma} w(\tau)-|v|^{\sigma} v(\tau) \|_{\mathbf{L}^{1, a}} d \tau \\
\quad+C \int_{0}^{t}\langle t-\tau\rangle^{\frac{a-1}{2}}\left\||w|^{\sigma} w(\tau)-|v|^{\sigma} v(\tau)\right\|_{\mathbf{L}^{1}} d \tau
\end{aligned}
$$




$$
\begin{aligned}
\leqslant C & \|w-v\|_{\mathbf{X}}\left(\|w\|_{\mathbf{X}}^{\sigma}+\|v\|_{\mathbf{X}}^{\sigma}\right)\left(\int_{0}^{t}\langle\tau\rangle^{-\frac{n}{2} \sigma+\frac{a}{2}}\langle t-\tau\rangle^{-\frac{1}{2}} d \tau\right. \\
& \left.+\int_{0}^{t}\langle\tau\rangle^{-\frac{n}{2} \sigma}\langle t-\tau\rangle^{\frac{a}{2}-\frac{1}{2}} d \tau\right) \leqslant C\langle t\rangle^{\frac{a}{2}}\|w-v\|_{\mathbf{X}}\left(\|w\|_{\mathbf{X}}^{\sigma}+\|v\|_{\mathbf{X}}^{\sigma}\right)
\end{aligned}
$$

при $\sigma>1 / n$, где мы воспользовались неравенством $\left\||w|^{\sigma} w(\tau)-|v|^{\sigma} v(\tau)\right\|_{\mathbf{L}^{1, a}} \leqslant$ $\|w-v\|_{\mathbf{X}}\left(\|w\|_{\mathbf{X}}^{\sigma}+\|v\|_{\mathbf{X}}^{\sigma}\right)\langle\tau\rangle^{-n \sigma / 2+a / 2}$. Таким образом, $\left\|\mathscr{G}_{2} \phi\right\|_{\mathbf{X}} \leqslant C\left\|_{\phi}\right\|_{\mathbf{Z}}$ и

$$
\left\|\int_{0}^{t} \mathscr{B}_{2} \mathscr{G}_{2}(t-\tau)(\mathscr{N}(w(\tau))-\mathscr{N}(v(\tau))) d \tau\right\|_{\mathbf{X}} \leqslant C\|w-v\|_{\mathbf{X}}\left(\|w\|_{\mathbf{X}}+\|v\|_{\mathbf{X}}\right)^{\sigma} .
$$

Следовательно, применяя теорему 1, мы видим, что существует единственное решение $u \in \mathbf{C}\left([0, \infty) ; \mathbf{L}^{1, a}\left(\mathbb{R}^{n}\right) \cap \mathbf{W}_{\infty}^{1}\left(\mathbb{R}^{n}\right)\right)$ задачи Коши $(24)$, удовлетворяющее оценкам

$$
\|u(t)\|_{\mathbf{W}_{\infty}^{1}} \leqslant C\langle t\rangle^{-n / 2} \quad \text { и } \quad\|u(t)\|_{\mathbf{L}^{1, a}} \leqslant C\langle t\rangle^{a / 2} .
$$

Для доказательства асимптотики решения воспользуемся теоремой 2. Благодаря оценкам леммы 2 имеем следующее представление для оператора Грина: $\mathscr{G}_{2}(t) u_{0}=\theta t^{-n / 2} \widetilde{G}_{0}\left(x t^{-1 / 2}\right)+O\left(t^{-(n+a) / 2}\left\|u_{0}\right\|_{\mathbf{L}^{1, a}}\right)$ при $t \rightarrow \infty$ равномерно по $x \in \mathbb{R}^{n}$, где $\widetilde{G}_{0}(\xi)=(4 \pi \alpha)^{-n / 2} e^{-|\xi|^{2} /(4 \alpha)}, \theta=\int_{\mathbb{R}^{n}} u_{0}(x) d x$ и $a \in(0,1]$. Также
ввиду леммы 2

$\left\|\mathscr{B}_{2} \mathscr{G}_{2}(t-\tau)(\lambda \cdot \nabla)|u|^{\sigma} u(\tau)\right\|_{\mathbf{L}^{\infty}} \leqslant C(t-\tau)^{-\frac{n}{2}-\frac{a}{2}}\left(\left\||u|^{\sigma} u(\tau)\right\|_{\mathbf{L}^{\infty}}+\left\||u|^{\sigma} u(\tau)\right\|_{\mathbf{L}^{1, a}}\right)$, поэтому в силу полученных выше оценок (25) для решения имеем

$$
\begin{gathered}
\left\|\int_{0}^{t}(\lambda \cdot \nabla) \mathscr{B}_{2} \mathscr{G}_{2}(t-\tau)|u|^{\sigma} u(\tau) d \tau\right\|_{\mathbf{L}^{\infty}} \leqslant C\|u\|_{\mathbf{X}}^{\sigma+1} \int_{0}^{\frac{t}{2}}(t-\tau)^{-\frac{n}{2}-\frac{a}{2}}\langle\tau\rangle^{-\frac{n}{2} \sigma} d \tau \\
+C\|u\|_{\mathbf{X}}^{\sigma+1} \int_{\frac{t}{2}}^{t}\langle\tau\rangle^{-\frac{n}{2}(\sigma+1)} d \tau \leqslant C\|u\|_{\mathbf{X}}^{\sigma+1}\langle t\rangle^{-\frac{n}{2}-\gamma},
\end{gathered}
$$

где $0<\gamma<\min (a / 2, n \sigma / 2-1)$. Таким образом, условия теоремы 2 выполнены и мы приходим к асимптотике $u(t, x)=\theta(4 \pi \alpha t)^{-n / 2} e^{-|x|^{2} /(4 \alpha t)}+O\left(t^{-n / 2-\gamma}\right)$ для решения задачи Коши (24). Теорема 4 доказана.

Применим также теоремы 1 и 2 к уравнению Соболева с конвективной нелинейностью

$$
\begin{gathered}
\partial_{t}(u-\Delta u)-\alpha \Delta u=\lambda \operatorname{div}\left(|\nabla u|^{\sigma} \nabla u\right), \quad x \in \mathbb{R}^{n}, \quad t>0, \\
u(0, x)=u_{0}(x), \quad x \in \mathbb{R}^{n},
\end{gathered}
$$

где $\alpha>0, \sigma>0, \lambda \in \mathbb{R}$ (см. уравнение (1) с $k=2, \mathscr{N}=\lambda \operatorname{div}\left(|\nabla u|^{\sigma} \nabla u\right), \sigma>0$, $\lambda \in \mathbb{R})$. Оказывается, что нелинейность $\mathscr{N}=\lambda \operatorname{div}\left(|\nabla u|^{\sigma} \nabla u\right)$ в уравнении (26) является слабой при любых $\sigma>0$.

Теорема 5. Пусть $\sigma>0$. Предположим, что $u_{0} \in \mathbf{L}^{1, a}\left(\mathbb{R}^{n}\right) \cap \mathbf{W}_{p}^{2}\left(\mathbb{R}^{n}\right)$, $a \in(0,1), p>(1+\sigma) /(2 \sigma)$ nри $n=1$ u $p>n$ при $n \geqslant 2$, причем норма $\left\|u_{0}\right\|_{\mathbf{W}_{p}^{2}\left(\mathbb{R}^{n}\right)}$ достаточно мала. Тогда существует единственное решение 
$u \in \mathbf{C}\left([0, \infty) ; \mathbf{W}_{p}^{2}\left(\mathbb{R}^{n}\right)\right)$ задачи Коши (26). Для этого решения справедлива асимптотика $u(t, x)=\theta(4 \pi \alpha t)^{-n / 2} e^{-|x|^{2} /(4 \alpha t)}+O\left(t^{-n / 2-\gamma}\right)$ nри $t \rightarrow \infty$ равномерно по $x \in \mathbb{R}^{n}$, где $\theta=\int_{\mathbb{R}^{n}} u_{0}(x) d x, \gamma=\min (1, \sigma, a)$.

ДокАзАтЕЛьство. Следуя методу доказательства теоремы 1 , рассмотрим шар $\mathbf{X}_{\rho}=\left\{u \in \mathbf{C}\left([0, \infty) ; \mathbf{W}_{p}^{2}\left(\mathbb{R}^{n}\right)\right):\|u\|_{\mathbf{Y}} \leqslant \rho\right\}$, где $\rho>0$ достаточно мало и

$$
\|u\|_{\mathbf{Y}}=\sup _{t>0} \sup _{0 \leqslant|\beta| \leqslant 2} \sup _{1 \leqslant q \leqslant p}\langle t\rangle^{\frac{n}{2}\left(1-\frac{1}{q}\right)+\frac{|\beta|}{2}}\left\|\partial^{\beta} u(t)\right\|_{\mathbf{L}^{q} .} .
$$

Для всех $v \in \mathbf{X}_{\rho}$ определим отображение $\mathscr{M}(v)$ формулой (17), которая с учетом (4) принимает вид

$$
\mathscr{M}(v)=\mathscr{G}_{1}(t) u_{0}+\int_{0}^{t} \mathscr{B}_{1} \mathscr{G}_{1}(t-\tau) \mathscr{N}(v(\tau)) d \tau,
$$

и докажем, что $\mathscr{M}(v) \in \mathbf{X}_{\rho}$. Имеем

$$
\begin{aligned}
\left\|\mathscr{B}_{1} \mathscr{N}(v)\right\|_{\mathbf{W}_{q}^{|\beta|}} & \leqslant C\|\mathscr{N}(v)\|_{\mathbf{L}^{q}} \leqslant C\|\Delta u(\tau)\|_{\mathbf{L}^{q}}\|\nabla v(\tau)\|_{\mathbf{W}_{p}^{1}}^{\sigma} \\
& \leqslant C\langle\tau\rangle^{-\frac{n}{2}\left(1-\frac{1}{q}\right)-1-\frac{\sigma}{2}-\frac{n \sigma}{2}\left(1-\frac{1}{p}\right)}\|v\|_{\mathbf{Y}}^{1+\sigma} .
\end{aligned}
$$

Ввиду первой оценки леммы 1 имеем $\left\|\mathscr{G}_{1} \phi\right\|_{\mathbf{Y}} \leqslant C\|\phi\|_{\mathbf{z}}$ и

$$
\begin{aligned}
& \langle t\rangle^{\frac{n}{2}\left(1-\frac{1}{q}\right)+\frac{|\beta|}{2}}\left\|\partial^{\beta} \int_{0}^{t} \mathscr{G}_{1}(t-\tau) \mathscr{B}_{1} \mathscr{N}(v) d \tau\right\|_{\mathbf{L}^{q}} \\
& \leqslant C\langle t\rangle^{\frac{n}{2}\left(1-\frac{1}{q}\right)+\frac{|\beta|}{2}} \int_{0}^{\frac{t}{2}}\langle t-\tau\rangle^{-\frac{n}{2}\left(1-\frac{1}{q}\right)-\frac{|\beta|}{2}}\left(\left\|\mathscr{B}_{1} \mathscr{N}(v)\right\|_{\mathbf{L}^{1}}+\left\|\mathscr{B}_{1} \mathscr{N}(v)\right\|_{\mathbf{W}_{p}^{|\beta|}}\right) d \tau \\
& +C\langle t\rangle^{\frac{n}{2}\left(1-\frac{1}{q}\right)+\frac{|\beta|}{2}} \int_{\frac{t}{2}}^{t}\langle t-\tau\rangle^{-\frac{|\beta|}{2}}\left\|\mathscr{B}_{1} \mathscr{N}(v)\right\|_{\mathbf{W}_{q}^{|\beta|}} d \tau \\
& \leqslant C\|v\|_{\mathbf{Y}}^{1+\sigma} \int_{0}^{\frac{t}{2}}\langle\tau\rangle^{-1-\frac{\sigma}{2}-\frac{n \sigma}{2}\left(1-\frac{1}{p}\right)} d \tau \\
& +C\|v\|_{\mathbf{Y}}^{1+\sigma} \int_{\frac{t}{2}}^{t}\langle t-\tau\rangle^{-\frac{|\beta|}{2}}\langle\tau\rangle^{\frac{|\beta|}{2}-1-\frac{\sigma}{2}-\frac{n \sigma}{2}\left(1-\frac{1}{p}\right)} d \tau \leqslant C\|v\|_{\mathbf{Y}}^{1+\sigma}
\end{aligned}
$$

для всех $t>0,0 \leqslant|\beta| \leqslant 2,1 \leqslant q \leqslant p$. Откуда следует, что $\mathscr{M}(v) \in \mathbf{X}_{\rho}$.

Покажем теперь, что $\mathscr{M}$ является сжимающим отображением в норме

$$
\|u\|_{\mathbf{X}}=\sup _{t>0} \sup _{|\beta|=0,1} \sup _{1 \leqslant q \leqslant p}\langle t\rangle^{\frac{n}{2}\left(1-\frac{1}{q}\right)+\frac{|\beta|}{2}}\left\|\partial^{\beta} u(t)\right\|_{\mathbf{L}^{q}} .
$$

Из теоремы вложения Соболева (см. [76], [77]) имеем $\|v\|_{\mathbf{L}^{\infty}} \leqslant C\|v\|_{\mathbf{W}_{p}^{1}}$ для $p>(1+\sigma) /(2 \sigma)$ при $n=1$ и для $p>n$ при $n \geqslant 2$, поэтому

$$
\begin{aligned}
& \left\|\mathscr{B}_{1}(\mathscr{N}(v)-\mathscr{N}(w))\right\|_{\mathbf{W}_{q}^{1}} \\
& \quad \leqslant C\|\nabla v(\tau)-\nabla w(\tau)\|_{\mathbf{L}^{q}}\left(\|\nabla v(\tau)\|_{\mathbf{W}_{p}^{1}}^{\sigma}+\|\nabla w(\tau)\|_{\mathbf{W}_{p}^{1}}^{\sigma}\right) \\
& \quad \leqslant C\langle\tau\rangle^{-\frac{n}{2}\left(1-\frac{1}{q}\right)-1-\frac{\sigma}{2}-\frac{n \sigma}{2}\left(1-\frac{1}{p}\right)}\|v-w\|_{\mathbf{X}}\left(\|v\|_{\mathbf{Y}}^{\sigma}+\|w\|_{\mathbf{Y}}^{\sigma}\right) .
\end{aligned}
$$


Отсюда следуют неравенства

$$
\begin{aligned}
& \langle t\rangle^{\frac{n}{2}\left(1-\frac{1}{q}\right)+\frac{|\beta|}{2} \|} \partial^{\beta} \int_{0}^{t} \mathscr{G}_{1}(t-\tau) \mathscr{B}_{1}(\mathscr{N}(v)-\mathscr{N}(w)) d \tau \|_{\mathbf{L}^{q}} \\
& \leqslant C\langle t\rangle^{\frac{n}{2}\left(1-\frac{1}{q}\right)+\frac{|\beta|}{2}} \int_{0}^{\frac{t}{2}}\langle t-\tau\rangle^{-\frac{n}{2}\left(1-\frac{1}{q}\right)-\frac{|\beta|}{2}} \\
& \quad \times\left(\left\|\mathscr{B}_{1}(\mathscr{N}(v)-\mathscr{N}(w))\right\|_{\mathbf{L}^{1}}+\left\|\mathscr{B}_{1}(\mathscr{N}(v)-\mathscr{N}(w))\right\|_{\mathbf{W}_{p}^{1}}\right) d \tau \\
& +C\langle t\rangle^{\frac{n}{2}\left(1-\frac{1}{q}\right)+\frac{|\beta|}{2}} \int_{\frac{t}{2}}^{t}\langle t-\tau\rangle^{-\frac{|\beta|}{2}}\left\|\mathscr{B}_{1}(\mathscr{N}(v)-\mathscr{N}(w))\right\|_{\mathbf{W}_{q}^{1}} d \tau \\
& \leqslant C\|v-w\|_{\mathbf{X}}\left(\|v\|_{\mathbf{Y}}^{\sigma}+\|w\|_{\mathbf{Y}}^{\sigma}\right)\left(\int_{0}^{\frac{t}{2}}\langle\tau\rangle^{-\frac{n \sigma}{2}-1-\frac{\sigma}{2}+\frac{n \sigma}{2 p}} d \tau\right. \\
& \left.+\int_{\frac{t}{2}}^{t}\langle t-\tau\rangle^{-\frac{|\beta|}{2}}\langle\tau\rangle^{\frac{|\beta|}{2}-1-\frac{\sigma}{2}-\frac{n \sigma}{2}\left(1-\frac{1}{p}\right)} d \tau\right) \leqslant C\|v-w\|_{\mathbf{X}}\left(\|v\|_{\mathbf{Y}}^{\sigma}+\|w\|_{\mathbf{Y}}^{\sigma}\right)
\end{aligned}
$$

для всех $t>0,|\beta|=0,1,1 \leqslant q \leqslant p$. Таким образом, $\mathscr{M}$ является сжимающим отображением в норме пространства $\mathbf{X}$; поэтому существует единственное решение $u \in \mathbf{C}\left([0, \infty) ; \mathbf{W}_{p}^{2}\left(\mathbb{R}^{n}\right)\right)$ задачи Коши $(26)$.

Докажем асимптотику для решения. Из второй оценки леммы 1 имеем $\mathscr{G}_{1}(t) u_{0}=\theta G_{0}(t, x)+O\left(t^{-(n+a) / 2}\left(\left\|u_{0}\right\|_{\mathbf{L}^{1, a}}+\left\|u_{0}\right\|_{\mathbf{L}^{\infty}}\right)\right)$, где $G_{0}(t, x)=(4 \pi \alpha t)^{-n / 2} \times$ $e^{-|x|^{2} /(4 \alpha t)}$. Для нелинейности $\mathscr{N}(u)=\lambda \operatorname{div}\left(|\nabla u|^{\sigma} \nabla u\right)$ в интегральном уравнении (17) найдем

$$
\left\|\int_{0}^{t} \mathscr{B}_{1} \mathscr{G}_{1}(t-\tau) \mathscr{N}(u(\tau)) d \tau\right\|_{\mathbf{L}^{\infty}} \leqslant C \int_{0}^{t}\left\|\mathscr{G}_{1}(t-\tau) \mathscr{B}_{1} \operatorname{div}\left(|\nabla u|^{\sigma} \nabla u\right)\right\|_{\mathbf{L}^{\infty}} d \tau .
$$

Поэтому в силу первой оценки леммы 1 имеем

$$
\begin{aligned}
& \langle t\rangle^{\frac{n+\gamma}{2}}\left\|\int_{0}^{t} \mathscr{B}_{1} \mathscr{G}_{1}(t-\tau) \mathscr{N}(u(\tau)) d \tau\right\|_{\mathbf{L}^{\infty}} \\
& \leqslant C\langle t\rangle^{\frac{n+\gamma}{2}} \int_{\frac{t}{2}}^{t}\langle t-\tau\rangle^{-\frac{1}{2}}\left(\left\|\mathscr{B}_{1}\left(|\nabla u|^{\sigma} \nabla u(\tau)\right)\right\|_{\left.\mathbf{L}^{\infty}+\left\|\mathscr{B}_{1}\left(|\nabla u|^{\sigma} \nabla u(\tau)\right)\right\|_{\mathbf{W}_{\infty}^{1}}\right) d \tau}\right. \\
& \quad+C\langle t\rangle^{\frac{n+\gamma}{2}} \int_{0}^{\frac{t}{2}}\langle t-\tau\rangle^{-\frac{n+1}{2}}\left(\left\|\mathscr{B}_{1}\left(|\nabla u|^{\sigma} \nabla u(\tau)\right)\right\|_{\mathbf{L}^{1}}+\left\|\mathscr{B}_{1}\left(|\nabla u|^{\sigma} \nabla u(\tau)\right)\right\|_{\mathbf{W}_{p}^{1}}\right) d \tau \\
& \leqslant C\langle t\rangle^{\frac{n+\gamma}{2}} \int_{\frac{t}{2}}^{t}\langle t-\tau\rangle^{-\frac{1}{2}}\|\nabla u(\tau)\|_{\mathbf{L}^{\infty}}^{1+\sigma} d \tau \\
& \quad+C\langle t\rangle^{\frac{n+\gamma}{2}} \int_{0}^{\frac{t}{2}}\langle t-\tau\rangle^{-\frac{n+1}{2}}\|\nabla u(\tau)\|_{\mathbf{L}^{\infty}}^{\sigma}\|\nabla u(\tau)\|_{\mathbf{L}^{1}} d \tau \\
& \leqslant C\|u\|_{\mathbf{Y}}^{1+\sigma}\langle t\rangle^{\frac{n+\gamma}{2}}\left(\int_{\frac{t}{2}}^{t}\langle t-\tau\rangle^{-\frac{1}{2}}\langle\tau\rangle^{-\frac{n+1}{2}(1+\sigma)} d \tau+\int_{0}^{\frac{t}{2}}\langle t-\tau\rangle^{-\frac{n+1}{2}}\langle\tau\rangle^{-\frac{1}{2}-\frac{n+1}{2} \sigma} d \tau\right) \\
& \leqslant C\|u\|_{\mathbf{Y}}^{1+\sigma},
\end{aligned}
$$

где $\gamma=\min (1, \sigma)$, при $p>(1+\sigma) /(2 \sigma)$ для $n=1$ и $p>n$ для $n \geqslant 2$. Откуда приходим к асимптотике для решения. Теорема 5 доказана. 
3.2. Большие начальные данные. В случае $\lambda<0$ в уравнении (23) можно избавиться от условия малости начальных возмущений. В работе [64] доказан следующий результат.

Теорема 6. Пусть $\sigma>2 / n, \lambda<0, n=1,2$. Предположим, что

$$
u_{0} \in \mathbf{W}_{\infty}^{2}\left(\mathbb{R}^{n}\right) \cap \mathbf{W}_{1}^{2}\left(\mathbb{R}^{n}\right) \cap \mathbf{L}^{1, a}\left(\mathbb{R}^{n}\right), \quad a \in(0,1] .
$$

Тогда существует единственное решение $u \in \mathbf{C}\left([0, \infty) ; \mathbf{L}^{1, a}\left(\mathbb{R}^{n}\right) \cap \mathbf{L}^{\infty}\left(\mathbb{R}^{n}\right)\right)$ задачи Коши (23). Для этого решения справедлива асимптотика $u(t, x)=$ $A t^{-n / 2} e^{-|x|^{2} /(4 \alpha t)}+O\left(t^{-n / 2-\gamma}\right)$ nри $t \rightarrow \infty$ равномерно по $x \in \mathbb{R}^{n}$, где $A-$ некоторая постоянная, $0<\gamma<\min (a / 2, n \sigma / 2-1)$.

Обратимся теперь к уравнению (24). Рассмотрим для простоты случай $\sigma \geqslant 1$. Имеет место следующий результат.

Теорема 7. Пусть $\sigma \geqslant 1, \lambda \in \mathbb{R}^{n}, n=1,2$. Предположим, ито

$$
u_{0} \in \mathbf{H}^{4}\left(\mathbb{R}^{n}\right) \cap \mathbf{W}_{1}^{2}\left(\mathbb{R}^{n}\right) \cap \mathbf{L}^{1, a}\left(\mathbb{R}^{n}\right), \quad a \geqslant 0 .
$$

Тогда существует единственное решение $u \in \mathbf{C}\left([0, \infty) ; \mathbf{H}^{4}\left(\mathbb{R}^{n}\right) \cap \mathbf{W}_{1}^{2}\left(\mathbb{R}^{n}\right) \cap\right.$ $\left.\mathbf{L}^{1, a}\left(\mathbb{R}^{n}\right)\right)$ задачи Коши (24). Для этого решения справедлива асимптотика $u(t, x)=\theta(4 \pi \alpha t)^{-n / 2} e^{-|x|^{2} /(4 \alpha t)}+O\left(t^{-n / 2-\gamma}\right)$ nри $t \rightarrow \infty$ равномерно по $x \in \mathbb{R}^{n}$, где $\theta=\int_{\mathbb{R}^{n}} u_{0}(x) d x, 0<\gamma<\min (a / 2, n \sigma / 2-1)$.

ДокАЗАтельство. Определим нормы $\|\phi\|_{p, q} \equiv\|\| \phi(t, x)\left\|_{\mathbf{L}^{q}\left(\mathbb{R}_{x}\right)}\right\|_{\mathbf{L}^{p}\left(\mathbb{R}_{t}^{+}\right)}$. Сначала докажем глобальное во времени существование решений $u \in \mathbf{C}([0, \infty)$; $\left.\mathbf{H}^{4}\left(\mathbb{R}^{n}\right) \cap \mathbf{W}_{1}^{2}\left(\mathbb{R}^{n}\right) \cap \mathbf{L}^{1, a}\left(\mathbb{R}^{n}\right)\right)$ задачи Коши (24), удовлетворяющих априорной оценке

$$
\left\|\left(1+\Delta^{2}\right) u\right\|_{\infty, 2}+\|\nabla(1-\Delta) u\|_{2,2} \leqslant C\left\|u_{0}\right\|_{\mathbf{H}^{2}} .
$$

Локальное существование решений $u \in \mathbf{C}\left([0, T] ; \mathbf{H}^{4}\left(\mathbb{R}^{n}\right) \cap \mathbf{W}_{1}^{2}\left(\mathbb{R}^{n}\right) \cap \mathbf{L}^{1, a}\left(\mathbb{R}^{n}\right)\right)$ задачи Коши (24) для некоторого $T>0$ легко установить, например, с помощью принципа сжимающих отображений. Умножим уравнение (24) на $2 u$ и проинтегрируем результат по всему пространству $x \in \mathbb{R}^{n}$, тогда

$$
\frac{d}{d t}\left(\|u(t)\|_{\mathbf{L}^{2}}^{2}+\|\Delta u(t)\|_{\mathbf{L}^{2}}^{2}\right)+2 \alpha\|\nabla u(t)\|_{\mathbf{L}^{2}}^{2}=0 .
$$

Затем, интегрируя по времени, получаем

$$
\|u(t)\|_{\mathbf{L}^{2}}^{2}+\|\Delta u(t)\|_{\mathbf{L}^{2}}^{2}+2 \alpha \int_{0}^{t}\|\nabla u(\tau)\|_{\mathbf{L}^{2}}^{2} d \tau \leqslant\left\|u_{0}\right\|_{\mathbf{H}^{2}}^{2}
$$

для всех $t \in[0, T]$. Аналогично, умножив уравнение $(24)$ на $2 \Delta^{2} u$ и проинтегрировав результат по всему пространству $x \in \mathbb{R}^{n}$, получаем

$$
\begin{aligned}
& \frac{d}{d t}\left(\|\Delta u\|_{\mathbf{L}^{2}}^{2}+\left\|\Delta^{2} u\right\|_{\mathbf{L}^{2}}^{2}\right)+2 \alpha\|\nabla \Delta u\|_{\mathbf{L}^{2}}^{2} \\
& \quad \leqslant C\|\nabla \Delta u\|_{\mathbf{L}^{2}}\left(\left\||u|^{\sigma} \Delta u\right\|_{\mathbf{L}^{2}}+\left\||u|^{\sigma-1}(\nabla u)^{2}\right\|_{\mathbf{L}^{2}}\right) .
\end{aligned}
$$


Интегрирование последнего неравенства по времени дает

$$
\|\Delta u\|_{\mathbf{L}^{2}}^{2}+\left\|\Delta^{2} u\right\|_{\mathbf{L}^{2}}^{2}+\alpha \int_{0}^{t}\|\nabla \Delta u\|_{\mathbf{L}^{2}}^{2} d \tau \leqslant C\left\|u_{0}\right\|_{\mathbf{H}^{4}}^{2}
$$

для всех $t \in[0, T]$. Пользуясь оценками леммы 2 и представлением (4), получаем неравенства

$$
\begin{aligned}
\|u\|_{\mathbf{W}_{1}^{2}} \leqslant C\left\|u_{0}\right\|_{\mathbf{W}_{1}^{2}}+C \int_{0}^{t}\left\|\nabla \mathscr{B}_{2} u^{2}(\tau)\right\|_{\mathbf{W}_{1}^{2}} d \tau \\
\leqslant C+C \int_{0}^{t}\|u(\tau)\|_{\mathbf{L}^{2}}^{2} d \tau \leqslant C(1+t) \\
\|u\|_{\mathbf{L}^{1, a}} \leqslant C\left\|u_{0}\right\|_{\mathbf{L}^{1, a}}+C \int_{0}^{t}\langle t\rangle^{\frac{a}{2}}\left\|\nabla \mathscr{B}_{2} u^{2}(\tau)\right\|_{\mathbf{L}^{1}} d \tau \\
\quad+C \int_{0}^{t}\left\|\nabla \mathscr{B}_{2} u^{2}(\tau)\right\|_{\mathbf{L}^{1, a}} d \tau \leqslant C\langle t\rangle^{\frac{a}{2}+1}+C \int_{0}^{t}\|u(\tau)\|_{\mathbf{L}^{1, a}} d \tau
\end{aligned}
$$

для всех $t \in[0, T]$, откуда по лемме Гронуолла следует, что

$$
e^{-C t}\left(\|u(t)\|_{\mathbf{L}^{1, a}}+\|u(t)\|_{\mathbf{W}_{1}^{2}}\right) \leqslant C
$$

для всех $t \in[0, T]$ с не зависящей от $T$ постоянной $C>0$. Таким образом, стандартной процедурой мы можем продолжить решение для всех $T>0$. При этом $\mathscr{B}_{2} \phi=\mathscr{F}^{-1}\left(1+|\xi|^{2 k}\right)^{-1} \hat{\phi}(\xi)$ задан своим ядром.

Применив к уравнению (24) оператор

$$
\mathscr{B}_{2} \phi=\mathscr{F}^{-1}\left(1+|\xi|^{4}\right)^{-1} \hat{\phi}(\xi)=\left(1+\Delta^{2}\right)^{-1} \phi,
$$

получим

$$
\partial_{t} u=\alpha \Delta u+(\lambda \cdot \nabla)|u|^{\sigma} u-\alpha \Delta^{3} \mathscr{B}_{2} u+(\lambda \cdot \nabla) \Delta^{2} \mathscr{B}_{2}|u|^{\sigma} u
$$

Чтобы оценить $\mathbf{L}^{1}(\mathbb{R})$-норму решения, умножим уравнение (28) на функцию $S(t, x)=\operatorname{sign}(u(t, x))$ и проинтегрируем результат по всему пространству $x \in \mathbb{R}^{n}$, тогда

$$
\begin{gathered}
\int_{\mathbb{R}^{n}} S(t, x) \partial_{t} u(t, x) d x=\alpha \int_{\mathbb{R}^{n}} S(t, x) \Delta u d x+\int_{\mathbb{R}^{n}} S(t, x)(\lambda \cdot \nabla)|u|^{\sigma} u d x \\
-\alpha \int_{\mathbb{R}^{n}} S(t, x) \Delta^{3} \mathscr{B}_{2} u d x+\int_{\mathbb{R}^{n}} S(t, x)(\lambda \cdot \nabla) \Delta^{2} \mathscr{B}_{2}|u|^{\sigma} u d x .
\end{gathered}
$$

Имеем

$$
\begin{gathered}
\int_{\mathbb{R}^{n}} u_{t}(t, x) S(t, x) d x=\frac{d}{d t}\|u(t)\|_{\mathbf{L}^{1}}, \\
\int_{\mathbb{R}^{n}} S(t, x)(\lambda \cdot \nabla)|u|^{\sigma} u d x=0, \quad \int_{\mathbb{R}^{n}} S(t, x) \Delta u d x \leqslant 0 .
\end{gathered}
$$


Для оставшихся членов в уравнении (29) докажем оценки

$$
\left|\int_{0}^{\infty} d t \int_{\mathbb{R}^{n}} S(t) \Delta^{3} \mathscr{B}_{2} u(t) d x\right| \leqslant C, \quad\left\|\Delta^{2} \mathscr{B}_{2}|u|^{\sigma} \nabla u\right\|_{1,1} \leqslant C .
$$

Перепишем уравнение (24) в интегральном виде:

$$
u(t)=\mathscr{G}_{2}(t) u_{0}+\int_{0}^{t} \mathscr{B}_{2} \mathscr{G}_{2}(t-\tau)(\lambda \cdot \nabla)|u|^{\sigma} u(\tau) d \tau,
$$

откуда, умножив на $\Delta^{3} \mathscr{B}_{2}$, получим

$$
\begin{aligned}
\Delta^{3} \mathscr{B}_{2} u(t)=\Delta^{3} \mathscr{B}_{2} \mathscr{G}_{2}(t) u_{0}+\int_{0}^{t-\nu(t)} \Delta^{3} \mathscr{B}_{2}^{2} \mathscr{G}_{2}(t-\tau)(\lambda \cdot \nabla)|u|^{\sigma} u(\tau) d \tau \\
+\int_{t-\nu(t)}^{t} \Delta^{3} \mathscr{B}_{2}^{2} \mathscr{G}_{2}(t-\tau)(\lambda \cdot \nabla)|u|^{\sigma} u(\tau) d \tau \equiv J_{1}+J_{2}+J_{3}
\end{aligned}
$$

где $\nu(t)=t^{2 / 3}$ при $t \geqslant 1$ и $\nu(t)=0$ при $t \in(0,1)$. Первое слагаемое в правой части (32) оценивается для всех $t>0$ с помощью леммы 2 следующим образом:

$$
\left\|J_{1}\right\|_{\mathbf{L}^{1}} \leqslant C\langle t\rangle^{-2}\left\|u_{0}\right\|_{\mathbf{W}_{1}^{2}} .
$$

Для второго слагаемого в правой части (32) имеем в силу первой оценки леммы 2

$$
\begin{aligned}
\mid \int_{0}^{T} d t & \int_{\mathbb{R}^{n}} S(t) \Delta^{3} \mathscr{B}_{2} u(t) d x \mid \leqslant \int_{0}^{T} d t\left\|J_{2}\right\|_{\mathbf{L}^{1}} \\
\leqslant & \int_{0}^{T} d t \int_{0}^{t-\nu(t)}\langle t-\tau\rangle^{-\frac{3}{2}}\left\||u|^{\sigma-1}(\nabla u)^{2}\right\|_{\mathbf{L}^{1}} d \tau \\
& \quad+C \int_{0}^{T} d t \int_{0}^{t-\nu(t)}\langle t-\tau\rangle^{-2}\left(\left\||u|^{\sigma-1}(\nabla u) \Delta u\right\|_{\mathbf{L}^{1}}+\left\||u|^{\sigma-1} u \nabla \Delta u\right\|_{\mathbf{L}^{1}}\right) d \tau \\
\leqslant & \int_{0}^{T} d t \int_{0}^{t-\nu(t)}\langle t-\tau\rangle^{-\frac{3}{2}}\|u\|_{\mathbf{L}^{\infty}}^{\sigma-1}\|\nabla u\|_{\mathbf{L}^{2}}^{2} d \tau \\
& +C \int_{0}^{T} d t \int_{0}^{t-\nu(t)}\langle t-\tau\rangle^{-2}\left(\|u\|_{\mathbf{L}^{\infty}}^{\sigma-1}\|\nabla u\|_{\mathbf{L}^{2}}\|\Delta u\|_{\mathbf{L}^{2}}\right. \\
& \left.+\|u\|_{\mathbf{L}^{\infty}}^{\sigma-1}\|u\|_{\mathbf{L}^{2}}\|\nabla \Delta u\|_{\mathbf{L}^{2}}\right) d \tau
\end{aligned}
$$

и в силу неравенств (27)

$$
\begin{aligned}
& \left|\int_{0}^{T} d t \int_{\mathbb{R}^{n}} S(t) \Delta^{3} \mathscr{B}_{2} u(t) d x\right| \\
& \leqslant C\left\|\left(1+\Delta^{2}\right) u\right\|_{\infty, 2}^{\sigma-1} \int_{0}^{T} d t\langle t\rangle^{-\frac{3}{2}} \int_{0}^{T}\|\nabla u(\tau)\|_{\mathbf{L}^{2}}\left(\|\nabla u(\tau)\|_{\mathbf{L}^{2}}+\|\Delta u(\tau)\|_{\mathbf{L}^{2}}\right) d \tau \\
& \quad+C\left\|\left(1+\Delta^{2}\right) u\right\|_{\infty, 2}^{\sigma} \int_{0}^{T} d t\langle t\rangle^{-\frac{8}{15}} \int_{\nu(t)}^{t} d \tau\langle\tau\rangle^{-\frac{6}{5}}\|\nabla \Delta u(t-\tau)\|_{\mathbf{L}^{2}} \\
& \leqslant C+C \int_{0}^{T} d \tau\langle\tau\rangle^{-\frac{6}{5}} \int_{0}^{T}\langle t\rangle^{-\frac{8}{15}}\|\nabla u(t-\tau)\|_{\mathbf{L}^{2}} d t \leqslant C
\end{aligned}
$$


Для оставшегося слагаемого $J_{3}$ в правой части (32) получаем

$$
\begin{gathered}
\int_{\mathbb{R}^{n}} d x S(t) J_{3}=\int_{\mathbb{R}^{n}} d x|u(t)| \int_{t-\nu(t)}^{t} d \tau \Delta^{3} \mathscr{B}_{2}^{2} \mathscr{G}_{2}(t-\tau) S(\tau)|u|^{\sigma-1}(\lambda \cdot \nabla) u(\tau) \\
+\int_{\mathbb{R}^{n}} d x S(t) \int_{t-\nu(t)}^{t} d \tau\left[\Delta^{3} \mathscr{B}_{2}^{2} \mathscr{G}_{2}(t-\tau), u(\tau)\right] S(\tau)|u|^{\sigma-1}(\lambda \cdot \nabla) u(\tau) \\
+\int_{\mathbb{R}^{n}} d x S(t) \int_{t-\nu(t)}^{t} d \tau(u(\tau, x)-u(t, x)) \Delta^{3} \mathscr{B}_{2}^{2} \mathscr{G}_{2}(t-\tau) S(\tau) \\
\quad \times|u|^{\sigma-1}(\lambda \cdot \nabla) u(\tau)=I_{1}+I_{2}+I_{3},
\end{gathered}
$$

где

$\left[\Delta^{3} \mathscr{B}_{2}^{2} \mathscr{G}_{2}(t-\tau), u(\tau)\right] \phi(\tau) \equiv \Delta^{3} \mathscr{B}_{2}^{2} \mathscr{G}_{2}(t-\tau)(u(\tau) \phi(\tau))-u(\tau) \Delta^{3} \mathscr{B}_{2}^{2} \mathscr{G}_{2}(t-\tau) \phi(\tau)$.

В первом слагаемом $I_{1}$ интегрирование по частям дает

$$
I_{1}=-\sum_{j=1}^{n} \int_{\mathbb{R}^{n}} d x \partial_{x_{j}}|u(t)| \int_{t-\nu(t)}^{t} d \tau \partial_{x_{j}} \Delta^{2} \mathscr{B}_{2}^{2} \mathscr{G}_{2}(t-\tau) S(t)|u|^{\sigma-1}(\lambda \cdot \nabla) u(\tau)
$$

откуда по неравенству Юнга ввиду (27) получаем

$$
\begin{aligned}
\int_{0}^{T}\left|I_{1}(t)\right| d t & \leqslant C \int_{0}^{T} d t\|\nabla u(t)\|_{\mathbf{L}^{2}} \int_{t-\nu(t)}^{t} \frac{d \tau}{\langle t-\tau\rangle^{2}}\|u(\tau)\|_{\mathbf{L}^{\infty}}^{\sigma-1}\|\nabla u(\tau)\|_{\mathbf{L}^{2}} \\
& \leqslant C \int_{0}^{T} d t\|\nabla u(t)\|_{\mathbf{L}^{2}} \int_{0}^{T} \frac{d \tau}{\langle\tau\rangle^{2}}\|\nabla u(t-\tau)\|_{\mathbf{L}^{2}} \leqslant C\|\nabla u\|_{2,2}^{2} \leqslant C .
\end{aligned}
$$

Для интеграла $I_{2}$ с помощью леммы 2 и неравенства Юнга найдем

$$
\begin{aligned}
\int_{0}^{T}\left|I_{2}(t)\right| d t & \leqslant \int_{0}^{T} d t \int_{0}^{t} d \tau\left\|\left[\Delta^{3} \mathscr{B}_{2}^{2} \mathscr{G}_{2}(t-\tau), u(\tau)\right] S(\tau)|u|^{\sigma-1}(\lambda \cdot \nabla) u(\tau)\right\|_{\mathbf{L}^{1}} \\
& \leqslant C \int_{0}^{T} d t \int_{0}^{t}\langle t-\tau\rangle^{-2}\|\nabla u(\tau)\|_{\mathbf{L}^{2}}^{2} d \tau \leqslant C\|\nabla u\|_{2,2}^{2} \leqslant C
\end{aligned}
$$

Чтобы оценить третье слагаемое $I_{3}$, запишем $u(t)-u(t-\tau)=\int_{0}^{\tau} u_{t}\left(t-t^{\prime}\right) d t^{\prime}$
и воспользуемся интегральным уравнением (31):

$$
u_{t}=\partial_{t} \mathscr{G}_{2}(t) u_{0}-\mathscr{B}_{2}\left((\lambda \cdot \nabla)|u|^{\sigma} u\right)-\int_{0}^{t} d \tau^{\prime} \partial_{t} \mathscr{G}_{2}\left(t-\tau^{\prime}\right) \mathscr{B}_{2}(\lambda \cdot \nabla)|u|^{\sigma} u\left(\tau^{\prime}\right)
$$

откуда благодаря лемме 2 имеем оценку

$$
\begin{gathered}
\|u(t)-u(t-\tau)\|_{\mathbf{L}^{2}} \leqslant C \int_{0}^{\tau} d t^{\prime}\left\langle t-t^{\prime}\right\rangle^{-2}+C \int_{0}^{\tau} d t^{\prime}\left\|u\left(t-t^{\prime}\right)\right\|_{\mathbf{L} \infty}\left\|\nabla u\left(t-t^{\prime}\right)\right\|_{\mathbf{L}^{2}} \\
+C \int_{0}^{\tau} d t^{\prime} \int_{0}^{t-t^{\prime}} d \tau^{\prime}\left\langle\tau^{\prime}\right\rangle^{-2}\left\|u\left(t-t^{\prime}-\tau^{\prime}\right)\right\|_{\mathbf{L}^{\infty}}\left\|\nabla u\left(t-t^{\prime}-\tau^{\prime}\right)\right\|_{\mathbf{L}^{2}} .
\end{gathered}
$$


Тогда получаем

$$
\begin{aligned}
& \int_{0}^{T}\left|I_{3}(t)\right| d t \leqslant \int_{0}^{T} d t \int_{0}^{\nu(t)} d \tau\|u(t)-u(t-\tau)\|_{\mathbf{L}^{1}} \\
& \times\left\|\Delta^{3} \mathscr{B}_{2}^{2} \mathscr{G}_{2}(\tau) S(t-\tau)|u|^{\sigma-1}(\lambda \cdot \nabla) u(t-\tau)\right\|_{\mathbf{L}^{\infty}} \\
& \leqslant C \int_{0}^{T} d t \int_{0}^{\nu(t)} d \tau\langle\tau\rangle^{-2}\|\nabla u(t-\tau)\|_{\mathbf{L}^{2}}\left(\int_{0}^{\tau} d t^{\prime}\left\langle t-t^{\prime}\right\rangle^{-2}\right. \\
&\left.\quad+\int_{0}^{\tau} d t^{\prime}\left\|\nabla u\left(t-t^{\prime}\right)\right\|_{\mathbf{L}^{2}}^{\frac{3}{2}}+\int_{0}^{\tau} d t^{\prime} \int_{0}^{t-t^{\prime}} d \tau^{\prime}\left\langle\tau^{\prime}\right\rangle^{-2}\left\|\nabla u\left(t-t^{\prime}-\tau^{\prime}\right)\right\|_{\mathbf{L}^{2}}^{\frac{3}{2}}\right) .
\end{aligned}
$$

Меняя порядок интегрирования и учитывая (27), приходим к оценке

$$
\begin{aligned}
\int_{0}^{T} & \left|I_{3}(t)\right| d t \leqslant \int_{0}^{T} d t^{\prime} \int_{t^{\prime}}^{T} d \tau\langle\tau\rangle^{-2} \int_{\tau}^{T} d t\|\nabla u(t-\tau)\|_{\mathbf{L}^{2}} \\
& \times\left(\left\langle t-t^{\prime}\right\rangle^{-2}+\left\|\nabla u\left(t-t^{\prime}\right)\right\|_{\mathbf{L}^{2}}^{\frac{3}{2}}+\int_{0}^{t-t^{\prime}} d \tau^{\prime}\left\langle\tau^{\prime}\right\rangle^{-2}\left\|\nabla u\left(t-t^{\prime}-\tau^{\prime}\right)\right\|_{\mathbf{L}^{2}}^{\frac{3}{2}}\right) \\
\leqslant & C \int_{0}^{T} d \tau\langle\tau\rangle^{-\frac{9}{8}} \int_{\tau}^{T} d t\|\nabla u(t-\tau)\|_{\mathbf{L}^{2}}^{2} \int_{0}^{T} d t^{\prime}\left\langle t^{\prime}\right\rangle^{-\frac{7}{8}}\left\|\nabla u\left(t-t^{\prime}\right)\right\|_{\mathbf{L}^{2}}^{1 / 2} \leqslant C .
\end{aligned}
$$

Оценки (36)-(38) вместе (35) дают

$$
\left.\left|\int_{\mathbb{R}^{n}} d x S(t) \int_{t-\nu(t)}^{t} \Delta^{3} \mathscr{B}_{2}^{2} \mathscr{G}_{2}(t-\tau)\right| u\right|^{\sigma}(\lambda \cdot \nabla) u(\tau) d \tau \mid \leqslant C .
$$

Собирая вместе неравенства (33), (34) и (39), приходим к первой оценке в (30).

Чтобы доказать вторую оценку в (30), запишем неравенство

$$
\left\|\Delta^{2} \mathscr{B}_{2}|u|^{\sigma} \nabla u\right\|_{\mathbf{L}^{1}} \leqslant C\|\nabla u\|_{\mathbf{L}^{2}}^{2}+\left\|u \mathscr{B}_{2} \Delta^{2} \nabla u\right\|_{\mathbf{L}^{1}}+\left\|\left[u, \mathscr{B}_{2}\right] \Delta^{2} \nabla u\right\|_{\mathbf{L}^{1}},
$$

где

$$
\left[u, \mathscr{B}_{2}\right] \Delta u=\frac{1}{2} \int_{\mathbb{R}} K(x-y)(u(t, x)-u(t, y)) \Delta u(t, y) d y
$$

С помощью интегрирования по частям и неравенства Коши получаем

$$
\begin{aligned}
\left\|\left[u, \mathscr{B}_{2}\right] \Delta^{2} \nabla u\right\|_{\mathbf{L}^{1}} & =\left\|\int_{\mathbb{R}^{n}} K(x-y)(u(t, x)-u(t, y)) \Delta^{2} \nabla u(t, y) d y\right\|_{\mathbf{L}^{1}} \\
\leqslant & \left\|\int_{\mathbb{R}^{n}} \nabla K(x-y)(u(t, x)-u(t, y)) \Delta^{2} u(t, y) d y\right\|_{\mathbf{L}^{1}} \\
& +\left\|\int_{\mathbb{R}^{n}} K(x-y) \nabla u(t, y) \Delta^{2} u(t, y) d y\right\|_{\mathbf{L}^{1}} \leqslant C\|\nabla u\|_{\mathbf{L}^{2}}^{2} .
\end{aligned}
$$


Поэтому $\left\|\left[u, \mathscr{B}_{2}\right] \Delta^{2} \nabla u\right\|_{1,1} \leqslant C$ для всех $t>0$. Ввиду интегрального уравнения (31) найдем

$$
\begin{gathered}
\left\|\mathscr{B}_{2} \Delta^{2} \nabla u(t)\right\|_{\mathbf{L}^{2}} \\
\leqslant\left\|\mathscr{B}_{2} \Delta^{2} \nabla \mathscr{G}_{2}(t) u_{0}\right\|_{\mathbf{L}^{2}}+\left\|\int_{0}^{t} \Delta^{2} \nabla \mathscr{B}_{2}^{2} \mathscr{G}_{2}(t-\tau)(\lambda \cdot \nabla)|u|^{\sigma} u(\tau) d \tau\right\|_{\mathbf{L}^{2}} \\
\leqslant C\langle t\rangle^{-2}\left\|u_{0}\right\|_{\mathbf{H}^{1}}+C \int_{0}^{t}\langle t-\tau\rangle^{-2}\|\nabla u(\tau)\|_{\mathbf{L}^{2}}^{2} d \tau \\
\quad+C \int_{0}^{t}\langle t-\tau\rangle^{-2}\|\nabla u(\tau)\|_{\mathbf{L}^{2}}^{1 / 2}\left\|\mathscr{B}_{2} \Delta^{2} u(\tau)\right\|_{\mathbf{L}^{2}} d \tau \\
\left\|\mathscr{B}_{2} \Delta^{2} u(\tau)\right\|_{\mathbf{L}^{2}} \\
\leqslant\left\|\mathscr{B}_{2} \Delta^{2} \mathscr{G}_{2}(t) u_{0}\right\|_{\mathbf{L}^{2}}+\left\|\int_{0}^{t} \Delta^{2} \mathscr{B}_{2}^{2} \mathscr{G}_{2}(t-\tau)(\lambda \cdot \nabla)|u|^{\sigma} u(\tau) d \tau\right\|_{\mathbf{L}^{2}} \\
\leqslant C\langle t\rangle^{-2}\left\|u_{0}\right\|_{\mathbf{L}^{2}}+C \int_{0}^{t}\langle t-\tau\rangle^{-2}\left(\|\nabla u(\tau)\|_{\mathbf{L}^{2}}^{2}+\|\nabla u(\tau)\|_{\mathbf{L}^{2}}^{\frac{3}{2}}\right) d \tau .
\end{gathered}
$$

Поэтому $\left\|\mathscr{B}_{2} \Delta^{2} u\right\|_{\frac{4}{3}, 2} \leqslant C$. Используя неравенство Юнга, из последних трех неравенств получаем

$$
\begin{aligned}
&\left\|\mathscr{B}_{2} \Delta^{2} \nabla u\right\|_{1,2} \leqslant C\left\|u_{0}\right\|_{\mathbf{H}^{1}}+C\left\|\int_{0}^{t}\langle t-\tau\rangle^{-2}\right\| \nabla u(\tau)\left\|_{\mathbf{L}^{2}}^{2} d \tau\right\|_{\mathbf{L}_{t}^{1}} \\
&+C\left\|\int_{0}^{t}\langle t-\tau\rangle^{-2}\right\| \nabla u(\tau)\left\|_{\mathbf{L}^{2}}^{1 / 2}\right\| \mathscr{B}_{2} \Delta^{2} u(\tau)\left\|_{\mathbf{L}^{2}} d \tau\right\|_{\mathbf{L}_{t}^{1}} \\
& \leqslant C\left\|u_{0}\right\|_{\mathbf{H}^{1}}+C\|\nabla u(\tau)\|_{2,2}^{2}+C\|\nabla u(\tau)\|_{2,2}^{1 / 2}\left\|\mathscr{B}_{2} \Delta^{2} u\right\|_{\frac{4}{3}, 2} \leqslant C .
\end{aligned}
$$

Так что справедлива вторая оценка в (30).

Теперь установим оптимальную оценку убывания

$$
\|u(t)\|_{\mathbf{L}^{p}} \leqslant C\langle t\rangle^{-\frac{n}{2}\left(1-\frac{1}{p}\right)}
$$

при $1 \leqslant p \leqslant 2$. Из $(29)$ найдем

$$
\begin{aligned}
\|u(t)\|_{\mathbf{L}^{1}} \leqslant\left\|u_{0}\right\|_{\mathbf{L}^{1}}+\left|\int_{0}^{t} d t \int_{\mathbb{R}^{n}} S(t) \Delta^{3} \mathscr{B}_{2} u d x\right| \\
+\left.\left|\int_{0}^{t} d t \int_{\mathbb{R}^{n}} S(t)(\lambda \cdot \nabla) \Delta^{2} \mathscr{B}_{2}\right| u\right|^{\sigma} u d x \mid \leqslant C .
\end{aligned}
$$

Поэтому для всех $t>0$ имеем

$$
\sup _{\xi \in \mathbb{R}^{n}}|\hat{u}(t, \xi)| \leqslant C\|u(t)\|_{\mathbf{L}^{1}} \leqslant\left\|u_{0}\right\|_{\mathbf{L}^{1}}+C \leqslant C,
$$

что дает оценку (42) при $p=1$.

Умножив уравнение (24) на $2 u$ и проинтегрировав результат по всем $x \in \mathbb{R}^{n}$, получим

$$
\frac{d}{d t}\left(\|u(t)\|_{\mathbf{L}^{2}}^{2}+\|\Delta u(t)\|_{\mathbf{L}^{2}}^{2}\right)=-2\|\nabla u(t)\|_{\mathbf{L}^{2}}^{2} .
$$


Используя теорему Планшереля и метод расщепления Фурье из работы [82], находим

$$
\begin{aligned}
\|\nabla u(t)\|_{\mathbf{L}^{2}}^{2} & =\|\xi \hat{u}(t)\|_{\mathbf{L}^{2}}^{2}=\int_{|\xi| \leqslant \delta}|\hat{u}(t, \xi)|^{2}|\xi|^{2} d \xi+\int_{|\xi| \geqslant \delta}|\hat{u}(t, \xi)|^{2}|\xi|^{2} d \xi \\
& \geqslant \delta^{2}\|u(t)\|_{\mathbf{L}^{2}}^{2}-2 \delta^{n+2} \sup _{|\xi| \leqslant \delta}|\hat{u}(t, \xi)|^{2}
\end{aligned}
$$

где $\delta>0$. Таким образом, из (43) получаем

$$
\frac{d}{d t}\|u(t)\|_{\mathbf{H}^{1}}^{2} \leqslant-2 \delta^{2}\|u(t)\|_{\mathbf{H}^{1}}^{2}+C \delta^{n+2} \sup _{|\xi| \leqslant \delta}|\hat{u}(t, \xi)|^{2} .
$$

Выберем теперь $2 \delta^{2}=(n+1)(1+t)^{-1}$ и сделаем замену $\|u(t)\|_{\mathbf{H}^{1}}^{2}=(1+t)^{-n-1} \times$ $W(t)$. Тогда, ввиду оценок $\|u(t)\|_{\mathbf{L}^{2}} \leqslant\left\|u_{0}\right\|_{\mathbf{H}^{1}}$ и $\|u(t)\|_{\mathbf{L}^{1}} \leqslant C$, получим из (44) неравенство $\frac{d}{d t} W(t) \leqslant C(1+t)^{n / 2}$. Откуда интегрированием по времени получаем

$$
W(t) \leqslant\left\|u_{0}\right\|_{\mathbf{H}^{1}}^{2}+C\left((1+t)^{\frac{n}{2}+1}-1\right),
$$

что дает оптимальную оценку убывания $\mathbf{L}^{2}$-нормы решения: $\|u(t)\|_{\mathbf{L}^{2}} \leqslant$ $C(1+t)^{-n / 4}$. Оценка (42) теперь следует из неравенства Гёльдера. Аналогично, пользуясь интегральным уравнением (31), устанавливаем оптимальные оценки убывания в $\mathbf{W}_{\infty}^{1}$ - и $\mathbf{L}^{1, a}$-нормах:

$$
\|u(t)\|_{\mathbf{W}_{\infty}^{1}} \leqslant C(1+t)^{-\frac{n}{2}}, \quad\|u(t)\|_{\mathbf{L}^{1, a}} \leqslant C(1+t)^{\frac{a}{2}} .
$$

Асимптотика $u(t, x)=\theta(4 \pi \alpha t)^{-n / 2} e^{-|x|^{2} /(4 \alpha t)}+O\left(t^{-n / 2-\gamma}\right)$ для решения задачи Коши (24) доказывается так же, как и при доказательстве теоремы 4 . Теорема 7 доказана.

Для уравнения (26) в случае $\lambda>0$ мы можем отказаться от требования малости начальных данных. Для простоты ограничимся случаем $n=1$.

Tеорема 8. Пусть $\sigma>0, \lambda>0, n=1$. Предположим, что $u_{0} \in \mathbf{L}^{1, a}(\mathbb{R}) \cap$ $\mathbf{H}^{2}(\mathbb{R}), a \in(0,1]$. Тогда существует единственное решение $u \in \mathbf{C}([0, \infty)$; $\left.\mathbf{H}^{2}(\mathbb{R})\right)$ задачи Коши (26). Для этого решения справедлива асимптотика $u(t, x)=\theta(4 \pi t)^{-1 / 2} e^{-x^{2} /(4 \alpha t)}+O\left(t^{-1 / 2-\gamma}\right)$ nри $t \rightarrow \infty$ равномерно по $x \in \mathbb{R}$, где $\theta=\int_{\mathbb{R}^{n}} u_{0}(x) d x, \gamma=\min (1, \sigma, a)$.

ДокАЗАтЕльство. Умножим уравнение (26), в одномерном случае имеющее вид $\partial_{t}\left(u-\partial_{x}^{2} u\right)-\alpha \partial_{x}^{2} u=\lambda \partial_{x}\left(\left|u_{x}\right|^{\sigma} u_{x}\right)$, на $2 u$ и проинтегрируем результат по $x \in \mathbb{R}$, тогда получим

$$
\frac{d}{d t}\left(\|u(t)\|_{\mathbf{L}^{2}}^{2}+\left\|u_{x}(t)\right\|_{\mathbf{L}^{2}}^{2}\right)=-2 \alpha\left\|u_{x}(t)\right\|_{\mathbf{L}^{2}}^{2}-2 \lambda\left\|u_{x}(t)\right\|_{\mathbf{L}^{\sigma+2}}^{\sigma+2} .
$$

Аналогично, умножив уравнение $(26)$ на $2 u_{x x}$ и проинтегрировав результат по $x \in \mathbb{R}$, найдем

$$
\frac{d}{d t}\left(\left\|u_{x}(t)\right\|_{\mathbf{L}^{2}}^{2}+\left\|u_{x x}(t)\right\|_{\mathbf{L}^{2}}^{2}\right)=-2 \alpha\left\|u_{x x}(t)\right\|_{\mathbf{L}^{2}}^{2}-2 \lambda(\sigma+1)\left\|\left|u_{x}\right|^{\sigma} u_{x x}^{2}\right\|_{\mathbf{L}^{1}} .
$$


Наконец, умножив уравнение $(26)$ на $2 x^{2} u_{x x}$ и проинтегрировав результат по $x \in \mathbb{R}$, получим

$$
\begin{aligned}
& \frac{d}{d t}\left(\left\|x u_{x}(t)\right\|_{\mathbf{L}^{2}}^{2}-\|u(t)\|_{\mathbf{L}^{2}}^{2}+\left\|x u_{x x}(t)\right\|_{\mathbf{L}^{2}}^{2}\right) \\
& \quad=-2 \alpha\left\|x^{2} u_{x x}(t)\right\|_{\mathbf{L}^{2}}^{2}-2 \lambda(\sigma+1)\left\|\left|u_{x}\right|^{\sigma} x^{2} u_{x x}^{2}\right\|_{\mathbf{L}^{1}} .
\end{aligned}
$$

В частности, мы видим, что нормы $\|u(t)\|_{\mathbf{H}^{2}}$ и $\left\|x u_{x}(t)\right\|_{\mathbf{H}^{1}}$ ограничены для всех $t>0$.

Теперь докажем грубую оценку убывания $\left\|u_{x}(t)\right\|_{\mathbf{H}^{1}} \leqslant C\langle t\rangle^{-1 / 4}$ при $t>0$. Используя теорему Планшереля и метод расщепления Фурье из работы [82], имеем

$$
\begin{aligned}
\left\|u_{x x}(t)\right\|_{\mathbf{L}^{2}}^{2} & =\left\|\xi^{2} \hat{u}(t)\right\|_{\mathbf{L}^{2}}^{2}=\int_{|\xi| \leqslant \delta}|\hat{u}(t, \xi)|^{2} \xi^{4} d \xi+\int_{|\xi| \geqslant \delta}|\hat{u}(t, \xi)|^{2} \xi^{4} d \xi \\
& \geqslant \delta^{2}\left\|u_{x}(t)\right\|_{\mathbf{L}^{2}}^{2}-2 \delta^{3} \sup _{|\xi| \leqslant \delta}|\xi \hat{u}(t, \xi)|^{2},
\end{aligned}
$$

где $\delta>0$. Таким образом, из (45) получаем неравенство

$$
\frac{d}{d t}\left\|u_{x}(t)\right\|_{\mathbf{H}^{1}}^{2} \leqslant-\alpha \delta^{2}\left\|u_{x}(t)\right\|_{\mathbf{H}^{1}}^{2}+4 \alpha \delta^{3} \sup _{|\xi| \leqslant \delta}|\xi \hat{u}(t, \xi)|^{2} .
$$

Выберем $\alpha \delta^{2}=2\left(t_{0}+t\right)^{-1}, t_{0}=\sqrt{2 / \alpha}$ и сделаем замену $\left\|u_{x}(t)\right\|_{\mathbf{H}^{1}}^{2}=\left(t_{0}+t\right)^{-2} \times$ $W(t)$. Заметим, что

$$
\sup _{|\xi| \leqslant \delta}|\xi \hat{u}(t, \xi)| \leqslant C\|\xi \hat{u}(t, \xi)\|_{\mathbf{H}^{1}} \leqslant C\left\|x u_{x}(t)\right\|_{\mathbf{L}^{2}}+C\left\|u_{x}(t)\right\|_{\mathbf{L}^{2}} \leqslant C .
$$

В силу (47) из (46) получим

$$
\frac{d}{d t} W(t) \leqslant C\left(t_{0}+t\right)^{\frac{1}{2}}
$$

Интегрирование (48) по времени дает $W(t) \leqslant\left\|\partial_{x} u_{0}\right\|_{\mathbf{H}^{1}}^{2}+C\left(\left(t_{0}+t\right)^{3 / 2}-1\right)$. Таким образом, получаем оценку убывания $\left\|u_{x}(t)\right\|_{\mathbf{H}^{1}} \leqslant C\langle t\rangle^{-1 / 4}$ для всех $t>0$. Далее, имеем

$$
\begin{aligned}
\left\|u_{x}(t)\right\|_{\mathbf{H}^{1}} \leqslant C & \langle t\rangle^{-3 / 4}\left(\left\|u_{0}\right\|_{\mathbf{H}^{2}}+\left\|u_{0}\right\|_{\mathbf{L}^{1}}\right) \\
& +C \int_{0}^{t}\left\|\partial_{x}^{2} \mathscr{G}(t-\tau) \mathscr{B}\left(\left|u_{x}\right|^{\sigma} u_{x}\right)\right\|_{\mathbf{H}^{1}} d \tau,
\end{aligned}
$$

где, в силу первой оценки леммы 1 ,

$$
\begin{aligned}
\left\|\partial_{x}^{2} \mathscr{G}(t-\tau) \mathscr{B}\left(\left|u_{x}\right|^{\sigma} u_{x}\right)\right\|_{\mathbf{H}^{1}} & \leqslant C\langle t-\tau\rangle^{-\frac{1}{2 r}-\frac{3}{4}}\left(\left\|u_{x}\right\|_{\mathbf{L}^{r(\sigma+1)}}^{\sigma+1}+\left\|\left|u_{x}\right|^{\sigma} u_{x}\right\|_{\mathbf{H}^{1}}\right) \\
& \leqslant C\langle t-\tau\rangle^{-1-\frac{\sigma}{4}}\left\|u_{x}(\tau)\right\|_{\mathbf{H}^{1}}^{\sigma+1} .
\end{aligned}
$$

Поэтому

$$
\left\|u_{x}(t)\right\|_{\mathbf{H}^{1}} \leqslant C\langle t\rangle^{-\frac{3}{4}}\left(\left\|u_{0}\right\|_{\mathbf{H}^{2}}+\left\|u_{0}\right\|_{\mathbf{L}^{1}}\right)+C \int_{0}^{t}\langle t-\tau\rangle^{-1-\frac{\sigma}{4}}\left\|u_{x}(\tau)\right\|_{\mathbf{H}^{1}}^{\sigma+1} d \tau .
$$

Подставляя в правую часть (50) оценку $\left\|u_{x}(t)\right\|_{\mathbf{H}^{1}} \leqslant C\langle t\rangle^{-1 / 4}$, находим, что $\left\|u_{x}(t)\right\|_{\mathbf{H}^{1}} \leqslant C\langle t\rangle^{-\frac{3}{4}}\left(\left\|u_{0}\right\|_{\mathbf{H}^{2}}+\left\|u_{0}\right\|_{\mathbf{L}^{1}}\right)+C \int_{0}^{t}\langle t-\tau\rangle^{-1-\frac{\sigma}{4}}\langle\tau\rangle^{-\frac{1}{4}-\frac{\sigma}{4}} d \tau \leqslant C\langle t\rangle^{-\frac{\gamma}{4}}$, 
где $\gamma=\min (3,1+\sigma)$. Подстановка в правую часть (50) полученного неравенства $\left\|u_{x}(t)\right\|_{\mathbf{H}^{1}} \leqslant C\langle t\rangle^{-1 / 4-\sigma / 4}$ дает

$$
\begin{aligned}
\left\|u_{x}(t)\right\|_{\mathbf{H}^{1}} \leqslant C & \langle t\rangle^{-\frac{3}{4}}\left(\left\|u_{0}\right\|_{\mathbf{H}^{2}}+\left\|u_{0}\right\|_{\mathbf{L}^{1}}\right) \\
& +C \int_{0}^{t}\langle t-\tau\rangle^{-1-\frac{\sigma}{4}}\langle\tau\rangle^{-\frac{\gamma}{4}(1+\sigma)} d \tau \leqslant C\langle t\rangle^{-\frac{\gamma}{4}},
\end{aligned}
$$

где $\gamma=\min \left(3,(1+\sigma)^{2}\right)$. Итерируя эти оценки, мы приходим к неравенству $\left\|u_{x}(t)\right\|_{\mathbf{H}^{1}} \leqslant C\langle t\rangle^{-\gamma / 4}$ с $\gamma=\min \left(3,(1+\sigma)^{k}\right)$. Так что мы можем гарантировать, что $\gamma=3$ после конечного числа итераций. Таким образом, получаем оптимальную оценку убывания $\left\|u_{x}(t)\right\|_{\mathbf{H}^{1}} \leqslant C\langle t\rangle^{-3 / 4}$.

Асимптотика решения $u(t, x)=\theta(4 \pi t)^{-1 / 2} e^{-x^{2} /(4 \alpha t)}+O\left(t^{-1 / 2-\gamma}\right)$ задачи Коши (26) доказывается так же, как и при доказательстве теоремы 5 . Теорема 8 доказана.

\section{4. Критическая неконвективная нелинейность}

4.1. Малые начальные данные. В этом разделе мы разовьем общий подход к изучению асимптотики при больших временах для решений эволюционных диссипативных уравнений с критической неконвективной нелинейностью, т. е. когда скорость убывания по времени линейных и нелинейных членов в уравнении совпадает. Мы покажем, что характер асимптотического поведения решений эволюционных уравнений с критической неконвективной нелинейностью отличается от соответствующего линейного случая логарифмической коррекцией в скорости убывания по времени. Рассмотрим задачу Коши

$$
\begin{gathered}
u_{t}+\mathscr{N}(u)+\mathscr{L} u=0, \quad x \in \mathbb{R}^{n}, \quad t>0, \\
u(0, x)=u_{0}(x), \quad x \in \mathbb{R}^{n},
\end{gathered}
$$

с критической неконвективной нелинейностью $\mathscr{N}(u)$ (см. определение $(52)$ ниже). Пусть $\mathbf{Z}$ - некоторое метрическое пространство функций на $\mathbb{R}^{n}$, также фиксируем полное метрическое пространство $\mathbf{X}$ функций на $[0, \infty) \times \mathbb{R}^{n}$. Как и выше, будем обозначать через $G_{0} \in \mathbf{X}$ асимптотическое ядро оператора Грина $\mathscr{G}$ в пространствах $\mathbf{X}$ и $\mathbf{Z}$ с соответствующим непрерывным линейным функционалом $f$ (см. определение (18) выше). Будем называть нелинейность $\mathscr{N}$ в уравнении (51) критической неконвективной, если выполнена оценка

$$
\int_{0}^{t} \operatorname{Re} f\left(\mathscr{N}\left(\theta G_{0}(\tau)\right)\right) d \tau \geqslant \eta \theta^{\sigma+1} \log (1+t)
$$

для всех $t>0, \theta>0$ с некоторыми положительными постоянными $\eta$ и $\sigma$. Смысл неконвективности состоит в том, что функционал от нелинейности отличен от нуля. А критичность заключается в относительно медленном убывании по времени, так что интеграл логарифмически расходится. Поскольку часто функционал $f$ задается интегралом по всему пространству: $f(\phi)=\int_{\mathbb{R}^{n}} \phi(x) d x$, то в этом случае типичным примером неконвективной нелинейности является степенная нелинейность $\mathscr{N}=\lambda|u|^{\sigma} u$, в то время как нелинейности, имеющие вид полной производной, $\mathscr{N}=\lambda \operatorname{div}\left(|\nabla u|^{\sigma} \nabla u\right)$ и $\mathscr{N}=(\lambda \cdot \nabla)|u|^{\sigma} u$, относятся к конвективному типу. 
Теперь мы установим глобальное существование и получим асимптотику при больших временах решений задачи Коши (51) с критической неконвективной нелинейностью $\mathscr{N}$. Определим функцию $g(t)=1+\eta \theta^{\sigma} \log (1+t)$ с некоторыми положительными постоянными $\eta, \theta$ и $\sigma$.

ТеОрема 9. Предположим, что линейный оператор $\mathscr{L}$ в (51) имеет нулевое среднее значение: $f(\mathscr{L} u)=0$ для всех $u \in \mathbf{X}$. Пусть нелинейность $\mathscr{N}(u)$ в (51) является критической неконвективной и такой, что

$$
e^{z} \mathscr{N}\left(u e^{-z}\right)=e^{-\sigma \operatorname{Re} z} \mathscr{N}(u)
$$

для любых $z \in \mathbb{C} u u \in \mathbf{X}$, где $\sigma>0$. Пусть начальнье возмущения $u_{0} \in \mathbf{Z}$ имеют малую норму $\left\|u_{0}\right\|_{\mathbf{z}} \leqslant \varepsilon u \theta \equiv\left|f\left(u_{0}\right)\right| \geqslant C \varepsilon>0$ при некотором $C>0$. Предположим, что при всех $t>0$ имеет место оценка

$$
\begin{aligned}
& |\nu(t) f(\mathscr{N}(v(t))-\mathscr{N}(w(t)))| \\
& \quad \leqslant C\{t\}^{-\alpha}\langle t\rangle^{-1}\|\nu(t)(v(t)-w(t))\|_{\mathbf{X}}\left(\|v\|_{\mathbf{X}}^{\sigma}+\|w\|_{\mathbf{X}}^{\sigma}\right)
\end{aligned}
$$

для любых $v, w \in \mathbf{X}, \nu(t)>0$, где $\alpha<1, \sigma>0$, а такюе оценка

$$
\begin{aligned}
\| g(t) & \int_{0}^{t} g^{-1}(\tau)|\mathscr{G}(t-\tau)(\mathscr{K}(v(\tau))-\mathscr{K}(w(\tau)))| d \tau \|_{\mathbf{X}} \\
& \leqslant C\|v-w\|_{\mathbf{X}}\left(\|v\|_{\mathbf{X}}+\|w\|_{\mathbf{X}}\right)^{\sigma}\left(1+\frac{\|v\|_{\mathbf{X}}+\|w\|_{\mathbf{X}}}{\theta}\right)
\end{aligned}
$$

для любых $v, w \in \mathbf{X} \operatorname{maкux,~что~} f(v)=f(w)=\theta>0$, где $\sigma>0$ и $\mathscr{K}(v)=$ $\mathscr{N}(v)-v \theta^{-1} f(\mathscr{N}(v))$. Тогда существует единственное глобальное решение $u \in \mathbf{X}$ задачи Коши (51). Это решение удовлетворяет оценке $\left\|g^{1 / \sigma} u\right\|_{\mathbf{X}} \leqslant C \varepsilon$. Более того, в предположении, что

$$
1+\frac{\sigma}{\theta} \int_{0}^{t} \operatorname{Re} f\left(\mathscr{N}\left(\theta G_{0}(\tau)\right)\right) d \tau=g(t)+O(\log g(t))
$$

при $t \rightarrow \infty$, справедлива асимптотическая ощенка

$$
\left\|\left(u-\theta g^{-\frac{1}{\sigma}} e^{i \psi} G_{0}\right) \frac{g^{1+\frac{1}{\sigma}}}{\log \log (4+t)}\right\|_{\mathbf{X}} \leqslant C,
$$

где $\psi(t)=\tilde{\eta} \log \log t+O(1)$ при $t \rightarrow \infty$ с некоторой постоянной $\tilde{\eta} \in \mathbb{R}$.

ДокАЗАТЕЛЬСтво. Как в работе [83], сделаем замену переменной $u(t, x)=$ $v(t, x) e^{-\varphi(t)+i \psi(t)}$ в уравнении (51). Тогда в силу условия (53) получаем следующее уравнение для новой функции $v(t, x)$ :

$$
v_{t}+\mathscr{L} v+e^{-\sigma \varphi} \mathscr{N}(v)-\left(\varphi^{\prime}-i \psi^{\prime}\right) v=0 .
$$

Выберем теперь вспомогательные функции $\varphi(t)$ и $\psi(t)$ из следующего условия:

$$
f\left(e^{-\sigma \varphi} \mathscr{N}(v)-\left(\varphi^{\prime}-i \psi^{\prime}\right) v\right)=0 .
$$

В силу предположения теоремы, $f(\mathscr{L} v)=0$, поэтому имеем закон сохранения

$$
\frac{d}{d t} f(v(t))=f\left(v_{t}(t)\right)=0
$$


Так что $f(v(t))=f\left(v_{0}\right)$ для всех $t>0$, где $v_{0}=v(0, x)$. Выберем начальные значения $\varphi(0)=0$ и $\psi(0)=\arg f\left(u_{0}\right)$, тогда $f(v(t))=f\left(v_{0}\right)=\left|f\left(u_{0}\right)\right|=\theta>0$ и

$$
\varphi^{\prime}=\frac{1}{\theta} e^{-\sigma \varphi} \operatorname{Re} f(\mathscr{N}(v)), \quad \psi^{\prime}=-\frac{1}{\theta} e^{-\sigma \varphi} \operatorname{Im} f(\mathscr{N}(v)) .
$$

Таким образом, получаем следующую задачу для новой функции $v(t, x)$ :

$$
\begin{gathered}
v_{t}+\mathscr{L} v=-e^{-\sigma \varphi}\left(\mathscr{N}(v)-\frac{v}{\theta} f(\mathscr{N}(v))\right), \quad x \in \mathbb{R}^{n}, \quad t>0, \\
v(0, x)=v_{0}(x) \equiv u_{0}(x) e^{-i \arg f\left(u_{0}\right)}, \quad x \in \mathbb{R}^{n} .
\end{gathered}
$$
Обозначим $h_{v}(t)=e^{\sigma \varphi(t)}$, тогда $\frac{d}{d t} h_{v}(t)=\frac{\sigma}{\theta} \operatorname{Re} f(\mathscr{N}(v)), h_{v}(0)=1$. Откуда,
интегрируя по времени, находим

$$
h_{v}(t)=1+\frac{\sigma}{\theta} \int_{0}^{t} \operatorname{Re} f(\mathscr{N}(v(\tau))) d \tau .
$$

Перепишем задачу (57) в виде интегрального уравнения

$$
v(t)=\mathscr{G}(t) v_{0}-\int_{0}^{t} \mathscr{G}(t-\tau) \mathscr{K}(v(\tau)) \frac{d \tau}{h_{v}(\tau)}
$$

с нелинейностью

$$
\mathscr{K}(v(\tau))=\mathscr{N}(v(\tau))-\frac{v(\tau)}{\theta} f(\mathscr{N}(v(\tau)))
$$

и функционалом

$$
h_{v}(t)=1+\frac{\sigma}{\theta} \int_{0}^{t} \operatorname{Re} f(\mathscr{N}(v(\tau))) d \tau
$$

Докажем существование решения $v(t, x)$ интегрального уравнения $(58)$ с помощью принципа сжимающих отображений. Определим отображение $\mathscr{M}(w)$ формулой

$$
\mathscr{M}(w)=\mathscr{G}(t) v_{0}-\int_{0}^{t} \mathscr{G}(t-\tau) \mathscr{K}(w(\tau)) \frac{d \tau}{h_{w}(\tau)},
$$

где

$$
h_{w}(t)=1+\frac{\sigma}{\theta} \int_{0}^{t} \operatorname{Re} f(\mathscr{N}(w(\tau))) d \tau
$$

для любой функции $w$ из множества

$$
\begin{aligned}
\mathbf{B}=\{w \in \mathbf{X}: & f(w)=\theta,\|w\|_{\mathbf{X}} \leqslant C \varepsilon \\
& \left.\left\|\log (2+t)\left(w-\theta G_{0}(t)\right)\right\|_{\mathbf{X}} \leqslant C \varepsilon, \sup _{t>0} \frac{g(t)}{h_{w}(t)} \leqslant 3\right\} .
\end{aligned}
$$

Проверим сначала, что отображение $\mathscr{M}$ преобразует множество В в себя. Поскольку $f(\mathscr{K}(w))=f(\mathscr{N}(w))-\theta^{-1} f(\mathscr{N}(w)) f(w)=0$, видим, что $f(\mathscr{M}(w))=$ $f\left(\mathscr{G}(t) v_{0}\right)=f\left(v_{0}\right)=\theta$. По определению асимптотического ядра (см. (18)) имеем

$$
\left\|\log (2+t)\left(\mathscr{G}(t) v_{0}-\theta G_{0}(t)\right)\right\|_{\mathbf{X}} \leqslant C\left\|\langle t\rangle^{\gamma}\left(\mathscr{G}(t) v_{0}-\theta G_{0}(t)\right)\right\|_{\mathbf{X}} \leqslant C\left\|v_{0}\right\|_{\mathbf{z}} \leqslant C \varepsilon .
$$


Поэтому из условий теоремы получаем

$$
\begin{aligned}
& \left\|\log (2+t) \mid \int_{0}^{t} \mathscr{G}(t-\tau) \mathscr{K}(w(\tau)) \frac{d \tau}{h_{w}(\tau)}\right\| \|_{\mathbf{X}} \\
& \leqslant 3\left\|\log (2+t) \int_{0}^{t} g^{-1}(\tau)|\mathscr{G}(t-\tau) \mathscr{K}(w(\tau))| d \tau\right\|_{\mathbf{X}} \\
& \leqslant \frac{C}{\eta \theta^{\sigma}}\|w\|_{\mathbf{X}}^{\sigma+1}\left(1+\frac{\|w\|_{\mathbf{X}}}{\theta}\right) \leqslant C \varepsilon .
\end{aligned}
$$

В частности, видим, что

$$
\begin{aligned}
\|\mathscr{M}(w)\|_{\mathbf{X}} & \leqslant\left\|\mathscr{G}(t) v_{0}\right\|_{\mathbf{x}}+\left\|\int_{0}^{t} \mathscr{G}(t-\tau) \mathscr{K}(w(\tau)) \frac{d \tau}{h_{w}(\tau)}\right\|_{\mathbf{X}} \\
& \leqslant C \varepsilon+C\left\|\int_{0}^{t} g^{-1}(\tau)|\mathscr{G}(t-\tau) \mathscr{K}(w(\tau))| d \tau\right\|_{\mathbf{X}} \leqslant C \varepsilon+C \varepsilon^{\sigma+1} \leqslant C \varepsilon,
\end{aligned}
$$

а также

$$
\begin{gathered}
\left\|\log (2+t)\left(\mathscr{M}(w)-\theta G_{0}(t)\right)\right\|_{\mathbf{X}} \leqslant\left\|\log (2+t)\left(\mathscr{G}(t) v_{0}-\theta G_{0}(t)\right)\right\|_{\mathbf{X}} \\
+\left\|\log (2+t) \int_{0}^{t} \mathscr{G}(t-\tau) \mathscr{K}(w(\tau)) \frac{d \tau}{h_{w}(\tau)}\right\|_{\mathbf{X}} \leqslant C \varepsilon .
\end{gathered}
$$

Остается установить оценку

$$
h_{\mathscr{M}(w)}(t)=1+\frac{\sigma}{\theta} \int_{0}^{t} \operatorname{Re} f(\mathscr{N}(\mathscr{M}(w))) d \tau \geqslant \frac{1}{3} g(t)
$$

для всех $t>0$. В силу условия (52) получаем

$$
\begin{aligned}
1+\frac{\sigma}{\theta} \int_{0}^{t} & \operatorname{Re} f(\mathscr{N}(\mathscr{M}(w))) d \tau=1+\frac{\sigma}{\theta} \int_{0}^{t} \operatorname{Re} f\left(\mathscr{N}\left(\theta G_{0}(\tau)\right)\right) d \tau \\
& +\frac{\sigma}{\theta} \int_{0}^{t} \operatorname{Re} f\left(\mathscr{N}(\mathscr{M}(w))-\mathscr{N}\left(\theta G_{0}(\tau)\right)\right) d \tau \\
\geqslant & \frac{1}{2} g(t)+R(t),
\end{aligned}
$$

где

$$
R(t)=\frac{\sigma}{\theta} \int_{0}^{t} \operatorname{Re} f\left(\mathscr{N}(\mathscr{M}(w))-\mathscr{N}\left(\theta G_{0}(\tau)\right)\right) d \tau .
$$

Благодаря оценке (54) имеем

$$
\begin{aligned}
|R(t)| \leqslant C\left\|\log (2+t)\left(\mathscr{M}(w)-\theta G_{0}(t)\right)\right\|_{\mathbf{X}}\left(\|\mathscr{M}(w)\|_{\mathbf{X}}^{\sigma}+\left\|\theta G_{0}\right\|_{\mathbf{X}}^{\sigma}\right) \\
\times \int_{0}^{t}\{\tau\}^{-\alpha}\langle\tau\rangle^{-1} \frac{d \tau}{\log (2+\tau)} \leqslant C \varepsilon^{\sigma+1} \log \log (4+t) .
\end{aligned}
$$

Поэтому ввиду (59b) находим, что для всех $t>0$

$$
h_{\mathscr{M}(w)}(t) \geqslant \frac{1}{2} g(t)-C \varepsilon^{\sigma+1} \log \log (4+t) \geqslant \frac{1}{3} g(t) .
$$


Таким образом, отображение $\mathscr{M}$ преобразует В в себя. Теперь с помощью (55) оценим разность $\mathscr{M}(v)-\mathscr{M}(w)$ :

$$
\begin{aligned}
\|\mathscr{M}(v)-\mathscr{M}(w)\|_{\mathbf{X}} \\
=\left\|\int_{0}^{t} \mathscr{G}(t-\tau)\left(\mathscr{K}(v(\tau)) \frac{1}{h_{v}(\tau)}-\mathscr{K}(w(\tau)) \frac{1}{h_{w}(\tau)}\right) d \tau\right\|_{\mathbf{X}} \\
\leqslant C\left\|\int_{0}^{t} g^{-1}(\tau)|\mathscr{G}(t-\tau)(\mathscr{K}(v(\tau))-\mathscr{K}(w(\tau)))| d \tau\right\|_{\mathbf{X}} \\
\quad+C\left\|\int_{0}^{t} g^{-1}(\tau)|\mathscr{G}(t-\tau) \mathscr{K}(w(\tau))| \frac{\left|h_{v}(\tau)-h_{w}(\tau)\right|}{g(\tau)} d \tau\right\|_{\mathbf{X}} \\
\leqslant C\|v-w\|_{\mathbf{X}}\left(\varepsilon^{\sigma}+\frac{1}{\theta}\left\|\int_{0}^{t} g^{-1}(\tau)|\mathscr{G}(t-\tau) \mathscr{K}(w(\tau))| d \tau\right\|_{\mathbf{X}}\right) \\
\leqslant C \varepsilon^{\sigma}\left(1+\frac{\varepsilon}{\theta}\right)\|v-w\|_{\mathbf{X}} \leqslant \frac{1}{2}\|v-w\|_{\mathbf{X}},
\end{aligned}
$$

где мы воспользовались следующей оценкой, вытекающей из (54) с $\nu(t)=1$ :

$$
\begin{aligned}
\frac{\left|h_{v}(\tau)-h_{w}(\tau)\right|}{g(\tau)} & \leqslant \frac{C}{\theta g(\tau)}\left|\int_{0}^{\tau} f(\mathscr{N}(v(t))-\mathscr{N}(w(t))) d t\right| \\
& \leqslant \frac{C \varepsilon^{\sigma} \log (1+\tau)}{\theta g(\tau)}\|v-w\|_{\mathbf{X}} \leqslant \frac{C}{\theta}\|v-w\|_{\mathbf{X}} .
\end{aligned}
$$

Таким образом, отображение $\mathscr{M}$ является сжимающим в В. Значит, существует единственное глобальное во времени решение $v \in \mathbf{B}$ задачи Коши (57), удовлетворяющее оценкам $\|v\|_{\mathbf{X}} \leqslant C \varepsilon,\left\|\log (2+t)\left(v-\theta G_{0}(t)\right)\right\|_{\mathbf{x}} \leqslant C \varepsilon$ и $h_{v}(t) \geqslant$ $g(t) / 3$. В силу соотношения $u(t, x)=v(t, x) e^{i \psi(t)} h_{v}^{-1 / \sigma}(t)$ получаем существование единственного глобального во времени решения задачи Коши (51), удовлетворяющего оценке убывания $\left\|g^{1 / \sigma} u\right\|_{\mathbf{x}} \leqslant C \varepsilon$. Установим асимптотику этого решения. Из условия (56) и представления (59а) имеем

$$
\begin{aligned}
h_{v}(t) & =1+\frac{\sigma}{\theta} \int_{0}^{t} \operatorname{Re} f(\mathscr{N}(v)) d \tau \\
& =1+\frac{\sigma}{\theta} \int_{0}^{t} \operatorname{Re} f\left(\mathscr{N}\left(\theta G_{0}(\tau)\right)\right) d \tau+\frac{\sigma}{\theta} \int_{0}^{t} \operatorname{Re} f\left(\mathscr{N}(v)-\mathscr{N}\left(\theta G_{0}(\tau)\right)\right) d \tau \\
& =g(t)+O(\log \log (4+t)) .
\end{aligned}
$$

Откуда по формулам $u(t, x)=e^{-\varphi(t)+i \psi(t)} v(t, x)=h_{v}^{-1 / \sigma}(t) e^{i \psi(t)} v(t, x)$ находим оценку

$$
\left\|\frac{g^{1+1 / \sigma}}{\log \log (4+t)}\left(u-\theta G_{0} e^{-i \psi} g^{-1 / \sigma}\right)\right\|_{\mathbf{X}} \leqslant C,
$$

так как

$$
\begin{aligned}
& \left\|\frac{g^{1+1 / \sigma}}{\log \log (4+t)}\left(\theta G_{0} e^{-i \psi}\left(h_{v}^{-1 / \sigma}-g^{-1 / \sigma}\right)\right)\right\|_{\mathbf{X}} \\
& \quad \leqslant C \sup _{t>0}\left|\frac{g^{1+1 / \sigma}}{\log \log (4+t)}\left(h_{v}^{-1 / \sigma}-g^{-1 / \sigma}\right)\right|\left\|G_{0}\right\|_{\mathbf{X}} \leqslant C .
\end{aligned}
$$


Интегрируя уравнение $\psi^{\prime}=-\theta^{-1} e^{-\sigma \varphi} \operatorname{Im} f(\mathscr{N}(v))$, имеем

$$
\psi(t)=\arg f\left(u_{0}\right)-\frac{1}{\theta} \int_{0}^{t} h_{v}^{-1}(\tau) \operatorname{Im} f(\mathscr{N}(v(\tau))) d \tau,
$$

откуда, используя асимптотику для $h_{v}$ и $v$, находим

$$
\begin{aligned}
\psi(t)=\arg & f\left(u_{0}\right)-\frac{1}{\theta} \int_{0}^{t} g^{-1}(\tau) \operatorname{Im} f\left(\mathscr{N}\left(\theta G_{0}(\tau)\right)\right) d \tau \\
+ & O\left(\int_{0}^{t}\{\tau\}^{-\alpha}\langle\tau\rangle^{-1} \frac{\log \log (4+\tau) d \tau}{\log ^{2}(2+\tau)}\right)=\tilde{\eta} \log \log t+O(1)
\end{aligned}
$$

при $t \rightarrow \infty$ с некоторой постоянной $\tilde{\eta} \in \mathbb{R}$. Теорема 9 доказана.

Следующий результат (см. работу [64]) получается применением теоремы 9 к уравнению Соболева со степенной нелинейностью (23). Обозначим

$$
\begin{gathered}
G_{0}(t, x)=(4 \pi \alpha t)^{-n / 2} e^{-|x|^{2} /(4 \alpha t)}, \quad \eta=(2 \pi \alpha)^{-1}(2 / n+1)^{-n / 2}, \\
g(t)=1+|\theta|^{2 / n} \eta \log (1+t), \quad \theta=\int_{\mathbb{R}^{n}} u_{0}(x) d x .
\end{gathered}
$$

Tеорема 10. Пусть $\lambda \theta<0, \sigma=2 / n$. Предположим, ито $u_{0} \in \mathbf{L}^{\infty}\left(\mathbb{R}^{n}\right) \cap$ $\mathbf{L}^{1, a}\left(\mathbb{R}^{n}\right), a \in(0,1)$, причем $\left\|u_{0}\right\|_{\mathbf{L}^{\infty}}+\left\|u_{0}\right\|_{\mathbf{L}^{1, a}} \leqslant \varepsilon, \lambda \theta \leqslant-C \varepsilon<0$. Тогда существует единственное решение $u \in \mathbf{C}\left([0, \infty) ; \mathbf{L}^{\infty}\left(\mathbb{R}^{n}\right) \cap \mathbf{L}^{1, a}\left(\mathbb{R}^{n}\right)\right)$ задачи Коши (23). Для этого решения справедлива асимптотика

$$
u(t, x)=\theta G_{0}(t, x) g^{-n / 2}(t)+O\left(\langle t\rangle^{-n / 2} g^{-n / 2-1}(t) \log \log t\right)
$$

при $t \rightarrow \infty$ равномерно по $x \in \mathbb{R}^{n}$.

Применим также теорему 9 к уравнению Соболева (24) в случае, когда начальные данные имеют нулевое среднее значение: $\int_{\mathbb{R}^{n}} u_{0}(x) d x=0$. Поскольку нелинейность в уравнении (24) имеет форму полной производной, то решение для всех времен также имеет нулевое среднее значение: $\int_{\mathbb{R}^{n}} u(t, x) d x=0$. Мы покажем, что в этом случае нелинейность $(\lambda \cdot \nabla)|u|^{\sigma} u$ в уравнении Соболева (24) ведет себя как неконвективная, так что асимптотика решений определяется первыми моментами. Вращением в пространственной плоскости мы можем преобразовать уравнение Соболева (24) к виду, в котором $\lambda=(1,0, \ldots, 0)$, таким образом рассмотрим задачу (сохраняя прежние обозначения для новых пространственных переменных и искомой функции)

$$
\begin{aligned}
\partial_{t}\left(u+\Delta^{2} u\right)-\alpha \Delta u & =\partial_{x_{1}}\left(|u|^{\sigma} u\right), \quad x \in \mathbb{R}^{n}, \quad t>0, \\
u(0, x) & =u_{0}(x), \quad x \in \mathbb{R}^{n},
\end{aligned}
$$

где $\alpha>0$. 
Введем пространство

$$
\mathbf{Z}=\left\{\phi \in \mathbf{L}^{1, a}\left(\mathbb{R}^{n}\right) \cap \mathbf{W}_{\infty}^{1}\left(\mathbb{R}^{n}\right): \int_{\mathbb{R}^{n}} \phi(x) d x=0 ; \int_{\mathbb{R}^{n}} x_{j} u_{0}(x) d x=0, j \neq 1\right\}
$$

и пространство

$$
\mathbf{X}=\left\{v \in \mathbf{C}\left([0, \infty) ; \mathbf{L}^{1, a}\left(\mathbb{R}^{n}\right) \cap \mathbf{W}_{\infty}^{1}\left(\mathbb{R}^{n}\right)\right): \int_{\mathbb{R}^{n}} v(t, x) d x=0 ;\|v\|_{\mathbf{X}}<\infty\right\}
$$

с нормой

$$
\|\phi\|_{\mathbf{X}}=\sup _{t \geqslant 0}\left(\langle t\rangle^{-\frac{a-1}{2}}\|\phi(t)\|_{\mathbf{L}^{1, a}}+\langle t\rangle^{\frac{n+1}{2}}\|\phi(t)\|_{\mathbf{W}_{\infty}^{1}}\right),
$$

где теперь $a \in(1,2]$. В так выбранных пространствах $\mathbf{X}$ и $\mathbf{Z}$ асимптотическое ядро оператора Грина $\mathscr{G}_{2}$ имеет вид

$$
G_{0}(t, x)=\frac{x_{1}}{(4 \pi \alpha)^{n / 2}(t+1)^{1+n / 2}} e^{-\frac{|x|^{2}}{4 \alpha(t+1)}}
$$

с функционалом $f(\phi) \equiv \int_{\mathbb{R}^{n}} \phi(x) x_{1} d x$. Обозначим $g(t)=1+\theta^{\sigma+1} \eta \log (1+t)$,

$$
\eta=|\lambda|(4 \pi)^{-\frac{n}{2}-\frac{n}{2(n+1)}} \int_{\mathbb{R}^{n}} e^{-\frac{|x|^{2}}{4}\left(1+\frac{1}{n+1}\right)}\left|x_{1}\right|^{\frac{1}{n+1}+1} d x>0
$$

и $\theta=\int_{\mathbb{R}^{n}} u_{0}(x) x_{1} d x$. В следующей теореме покажем, что нелинейность является критической при $\sigma=(n+1)^{-1}$.

Tеорема 11. Пусть $\sigma=(n+1)^{-1}, n=1,2,3$. Предположим, что $u_{0} \in$ $\mathbf{L}^{1, a}\left(\mathbb{R}^{n}\right) \cap \mathbf{W}_{\infty}^{1}\left(\mathbb{R}^{n}\right), a \in(1,2]$, причем норма $\left\|u_{0}\right\|_{\mathbf{L}^{1, a}}+\left\|u_{0}\right\|_{\mathbf{W}_{\infty}^{1}}=\varepsilon$ достаточно мала. Пусть $\int_{\mathbb{R}^{n}} u_{0}(x) d x=0, \int_{\mathbb{R}^{n}} x_{j} u_{0}(x) d x=0, j \neq 1, a \theta=\int_{\mathbb{R}^{n}} x_{1} u_{0}(x) d x \geqslant$ $C \varepsilon>0$ с некоторой постоянной $C>0$. Тогда существует единственное решение $и \in \mathbf{C}\left([0, \infty) ; \mathbf{L}^{1, a}\left(\mathbb{R}^{n}\right) \cap \mathbf{W}_{\infty}^{1}\left(\mathbb{R}^{n}\right)\right)$ задачи Коши (61). Для этого решения справедлива асимптотика

$$
u(t, x)=\theta G_{0}(t, x) g^{-\frac{n+1}{2}}(t)+O\left(t^{-\frac{n+1}{2}}(\log t)^{-\frac{n}{2}-1} \log \log t\right)
$$

при $t \rightarrow \infty$ равномерно по $x \in \mathbb{R}^{n}$.

ДокАЗАТЕЛЬСтво. Рассмотрим пространство

$$
\mathbf{Y}=\left\{v \in \mathbf{C}\left([0, \infty) ; \mathbf{L}^{1, a}\left(\mathbb{R}^{n}\right) \cap \mathbf{W}_{\infty}^{1}\left(\mathbb{R}^{n}\right)\right): \int_{\mathbb{R}^{n}} v(t, x) d x=0 ;\|v\|_{\mathbf{Y}}<\infty\right\}
$$

с нормой

$$
\|v\|_{\mathbf{Y}}=\sup _{t>0}\left(\langle t\rangle^{1-\frac{a-1}{2}}\|v(t)\|_{\mathbf{L}^{1, a}}+\langle t\rangle^{1+\frac{n+1}{2}}\|v(t)\|_{\mathbf{L}^{\infty}}\right) .
$$

Будем говорить, что нормы пространств $\mathbf{X}$ и $\mathbf{Y}$ согласованы по отношению к оператору $\mathscr{G}_{2}$, если для любой функции $\phi \in \mathbf{Y}$ такой, что $f(\phi)=0$, справедливо неравенство

$$
\left\|\log (2+t) \int_{0}^{t} \mathscr{G}(t-\tau) \phi(\tau) \frac{d \tau}{\log (2+\tau)}\right\|_{\mathbf{X}} \leqslant C\|\phi\|_{\mathbf{Y}} .
$$


Покажем, что нормы пространств $\mathbf{X}$ и $\mathbf{Y}$ согласованы по отношению к оператору $\mathscr{G}_{2}$. Ввиду оценки $\langle t\rangle^{-(a-1) / 4} \leqslant C g^{-1}(t)$ получим

$$
\begin{gathered}
\left\|\int_{0}^{t} g^{-1}(\tau) \mathscr{G}(t-\tau) \phi(\tau) d \tau\right\|_{\mathbf{L}^{\infty}} \\
\leqslant C \int_{0}^{\sqrt{t}}(t-\tau)^{-\frac{n}{2}-\frac{a}{2}}\langle\tau\rangle^{\frac{a-1}{2}-1} d \tau \sup _{\tau>0}\langle\tau\rangle^{1-\frac{a-1}{2}}\|\phi(\tau)\|_{\mathbf{L}^{1, a}} \\
\quad+C g^{-1}(t) \int_{\sqrt{t}}^{\frac{t}{2}}(t-\tau)^{-\frac{n}{2}-\frac{a}{2}}\langle\tau\rangle^{\frac{a-1}{2}-1} d \tau \sup _{\tau>0}\langle\tau\rangle^{1-\frac{a-1}{2}}\|\phi(\tau)\|_{\mathbf{L}^{1, a}} \\
\quad+C g^{-1}(t) \int_{\frac{t}{2}}^{t} \tau^{-\frac{n+1}{2}-1} d \tau \sup _{\tau>1} \tau^{1+\frac{n+1}{2}}\|\phi(\tau)\|_{\mathbf{L}^{\infty}} \\
\leqslant C t^{-\frac{n+1}{2}}\left(t^{-\frac{a-1}{4}}+g^{-1}(t)\right)\|\phi\|_{\mathbf{Y}} \leqslant C g^{-1}(t) t^{-\frac{n+1}{2}}\|\phi\|_{\mathbf{Y}}
\end{gathered}
$$

для всех $t>0$. Аналогично имеем

$$
\begin{gathered}
\left\|\int_{0}^{t} g^{-1}(\tau) \mathscr{G}(t-\tau) \phi(\tau) d \tau\right\|_{\mathbf{L}^{1, a}} \leqslant C \int_{0}^{\sqrt{t}}\langle\tau\rangle^{\frac{a-1}{2}-1} d \tau \sup _{\tau>0}\langle\tau\rangle^{1-\frac{a-1}{2}}\|\phi(\tau)\|_{\mathbf{L}^{1, a}} \\
+C g^{-1}(t) \int_{\sqrt{t}}^{t}\langle\tau\rangle^{\frac{a-1}{2}-1} d \tau \sup _{\tau>1} \tau^{1-\frac{a-1}{2}}\|\phi(\tau)\|_{\mathbf{L}^{1, a}} \\
\leqslant C \varepsilon\left(t^{\frac{a-1}{4}}+g^{-1}(t) t^{\frac{a-1}{2}}\right)\|\phi\|_{\mathbf{Y}} \leqslant C g^{-1}(t) t^{\frac{a-1}{2}}\|\phi\|_{\mathbf{Y}}
\end{gathered}
$$

для всех $t>0$. Следовательно, нормы пространств $\mathbf{X}$ и $\mathbf{Y}$ согласованы по отношению к оператору $\mathscr{G}_{2}$, так что условие (55) имеет место. Далее, прямое вычисление дает

$$
\begin{aligned}
\int_{0}^{t} \operatorname{Re} & f\left(\mathscr{N}\left(\theta G_{0}(\tau)\right)\right) d \tau=|\lambda| \theta^{\sigma+1} \int_{0}^{t} \int_{\mathbb{R}^{n}}\left|G_{0}(\tau, x)\right|^{\sigma+1} d x d \tau \\
= & |\lambda| \theta^{\sigma+1}(4 \pi)^{-\frac{n}{2}(1+\sigma)} \int_{0}^{t} \int_{\mathbb{R}^{n}} e^{-\frac{|x|^{2}}{4(\tau+1)}(1+\sigma)}\left|x_{1}\right|^{\sigma+1} d x(\tau+1)^{-(1+\sigma)\left(1+\frac{n}{2}\right)} d \tau \\
= & |\lambda| \theta^{\sigma+1}(4 \pi)^{-\frac{n}{2}-\frac{n}{2(n+1)}} \int_{\mathbb{R}^{n}} e^{-\frac{|x|^{2}}{4}\left(1+\frac{1}{n+1}\right)}\left|x_{1}\right|^{\frac{1}{n+1}+1} d x \int_{0}^{t} \frac{d \tau}{1+\tau} \\
= & \theta^{\sigma+1} \eta \int_{0}^{t} \frac{d \tau}{1+\tau}=\eta \theta^{\sigma+1} \log (1+t),
\end{aligned}
$$

поэтому условия (54) и (56) выполнены (условие (56) выполнено в силу оценок леммы 2). Применение теоремы 9 приводит нас к результату теоремы 11.

4.2. Большие начальные данные. Рассмотрим уравнение типа Соболева со степенной нелинейностью (23). Если $\lambda<0$, то мы можем ослабить требование малости начальных возмущений $u_{0}(x)$ в критическом случае $\sigma=2 / n$, однако в этом случае асимптотический результат имеет альтернативный характер. Обозначим

$$
G_{0}(t, x)=(4 \pi \alpha t)^{-n / 2} e^{-|x|^{2} /(4 \alpha t)}, \quad \eta=(4 \pi)^{-1}(1+2 / n)^{-n / 2} .
$$

В работе [65] доказан следующий результат. 
Tеорема 12. Пусть $\sigma=2 / n, \lambda<0$. Предположим, что $u_{0} \in \mathbf{W}_{\infty}^{2} \cap \mathbf{W}_{1}^{2,1} \cap$ $\mathbf{H}^{2, k}\left(\mathbb{R}^{n}\right) \cap \mathbf{C}\left(\mathbb{R}^{n}\right)$ при $k>6+3 n$. Тогда задача Коши (23) имеет единственное глобальное во времени решение $u \in \mathbf{C}\left([0, \infty) ; \mathbf{W}_{\infty}^{2} \cap \mathbf{H}^{2, k}\left(\mathbb{R}^{n}\right)\right)$. Более того, это решение и может иметь только одну из следующих асимптотик при $t \rightarrow \infty$ : либо равномерно по $x \in \mathbb{R}^{n}$

$$
u(t, x)=\left(\frac{n}{2 \eta}\right)^{\frac{n}{2}} G_{0}(t, x) \log ^{-\frac{n}{2}} t+O\left(t^{-\frac{n}{2}} \log ^{-\frac{n}{2}-\gamma} t\right), \quad \text { где } 0<\gamma<\frac{1}{n},
$$

либо $и(t)=O\left(t^{-n / 2} \log ^{-n / 2-1} t\right)$.

\section{5. Критическая конвективная нелинейность}

5.1. Малые начальные данные. В этом разделе мы изучим асимптотику при больших временах решений уравнений типа Соболева с критической нелинейностью конвективного типа. Мы покажем, что в этом случае характер асимптотики определяется автомодельными решениями. Рассмотрим задачу Коши

$$
\begin{gathered}
u_{t}+\mathscr{N}(u)+\mathscr{L} u=0, \quad x \in \mathbb{R}^{n}, \quad t>0, \\
u(0, x)=u_{0}(x), \quad x \in \mathbb{R}^{n},
\end{gathered}
$$

с критической нелинейностью $\mathscr{N}(u)$ конвективного типа (см. определение $(64)$ ниже). Пусть $\mathbf{Z}$ - некоторое метрическое пространство функций на $\mathbb{R}^{n}$, также фиксируем полное метрическое пространство $\mathbf{X}$ функций на $[0, \infty) \times \mathbb{R}^{n}$. Как и ранее, будем обозначать через $G_{0} \in \mathbf{X}$ асимптотическое ядро оператора Грина $\mathscr{G}$ в пространствах $\mathbf{X}, \mathbf{Z}$ с соответствующим непрерывным линейным функционалом $f$ (см. определение (18) выше).

Назовем оператор

$$
\mathscr{G}_{0}(t) \phi=t^{-\frac{n}{2}} \int_{\mathbb{R}^{n}} \widetilde{G}_{0}\left((x-y) t^{-\frac{1}{2}}\right) \phi(y) d y
$$

автомодельным асимптотическим оператором для оператора Грина $\mathscr{G}$ в пространствах X, Z, если выполнена оценка

$$
\left\|\mathscr{G}_{0}(t) \phi\right\|_{\mathbf{X}}+\left\|\langle t\rangle^{\gamma}\left(\mathscr{G}(t)-\mathscr{G}_{0}(t)\right) \phi\right\|_{\mathbf{X}} \leqslant C\|\phi\|_{\mathbf{Z}}
$$

для всех $\phi \in \mathbf{Z}$ с некоторым $\gamma>0$. Будем предполагать также, что асимптотическое ядро имеет автомодельный вид $G_{0}(t, x)=t^{-n / 2} \widetilde{G}_{0}\left(x t^{-1 / 2}\right)$, а линейный непрерывный функционал $f$ имеет свойство автомодельности

$$
f\left(t^{-\frac{n}{2}} \phi\left(x t^{-\frac{1}{2}}\right)\right)=f(\phi)
$$

для всех $t>0$ и $\phi \in \mathbf{Z}$. Введем теперь банахово пространство $\mathbf{Q}$ функций, заданных на $\mathbb{R}^{n}$, такое, что норма $\mathbf{Q}$ индуцируется нормой пространства $\mathbf{X}$, а именно, имеет место соотношение

$$
\|\phi\|_{\mathbf{Q}}=\left\|t^{-\frac{n}{2}} \phi\left(x t^{-\frac{1}{2}}\right)\right\|_{\mathbf{X}} .
$$


Будем называть нелинейность $\mathscr{N}$ в уравнении (62) критической конвективной, если $f(\mathscr{N}(u))=0$ для всех $u \in \mathbf{X}$ и

$$
t^{-\frac{n}{2}} \int_{0}^{1} \mathscr{G}_{0}\left(1-z, x t^{-\frac{1}{2}}\right) \mathscr{N}\left(z^{-\frac{n}{2}} \phi\left(x z^{-\frac{1}{2}}\right)\right) d z=\int_{0}^{t} \mathscr{G}_{0}(t-\tau, x) \mathscr{N}\left(\tau^{-\frac{n}{2}} \phi\left(x \tau^{-\frac{1}{2}}\right)\right) d \tau
$$

для любой $\phi \in \mathbf{Q}$.

Сначала установим существование автомодельных решений уравнения (62).

Лемма 3. Пусть оператор $\mathscr{G}_{0}(t)$ является автомодельным асимптотическим оператором для оператора Грина $\mathscr{G}$ в пространствах $\mathbf{X}, \mathbf{Z}$ и выполнено условие (63). Пусть нелинейность $\mathscr{N}$ в уравнении (62) является критической конвективной. Предположим также, что выполнено неравенство

$$
\left\|\int_{0}^{t} \mathscr{G}_{0}(t-\tau)(\mathscr{N}(v(\tau))-\mathscr{N}(w(\tau))) d \tau\right\|_{\mathbf{X}} \leqslant C\|v-w\|_{\mathbf{X}}\left(\|v\|_{\mathbf{X}}+\|w\|_{\mathbf{X}}\right)^{\sigma}
$$

для любых $v, w \in \mathbf{X}$. Тогда для достаточно малого $\theta(|\theta|>0)$ существует единственное решение $V \in \mathbf{Q}$ интегрального уравнения

$$
V=\theta \widetilde{G}_{0}-\int_{0}^{1} \mathscr{G}_{0}(1-z) \mathscr{N}\left(z^{-\frac{n}{2}} V\left(x z^{-\frac{1}{2}}\right)\right) d z
$$

maкое, что $\|V\|_{\mathbf{Q}} \leqslant C|\theta|$.

ДокАзАТЕЛЬство. Применим принцип сжимающих отображений. Определим отображение

$$
\mathscr{R}(V)=\theta \widetilde{G}_{0}-\int_{0}^{1} \mathscr{G}_{0}(1-z) \mathscr{N}\left(z^{-\frac{n}{2}} V\left(x z^{-\frac{1}{2}}\right)\right) d z
$$

для любой $V \in \mathbf{Q}_{\rho}$, где $\mathbf{Q}_{\rho}=\left\{V \in \mathbf{Q}:\|V\|_{\mathbf{Q}} \leqslant \rho\right\}$ и $C|\theta| \leqslant \rho$, а $\rho>0$ достаточно мало. Проверим, что $\mathscr{R}$ преобразует $\mathbf{Q}_{\rho}$ в себя. Обозначим $v(t, x)=$ $t^{-n / 2} V\left(x t^{-1 / 2}\right)$. В силу свойства (64) и связи норм пространств $\mathbf{Q}$ и $\mathbf{X}$ имеем

$$
\begin{aligned}
\|\mathscr{R}(V)\|_{\mathbf{Q}} & \leqslant\left\|\theta \widetilde{G}_{0}\right\|_{\mathbf{Q}}+\left\|\int_{0}^{1} \mathscr{G}_{0}(1-z, \xi) \mathscr{N}\left(z^{-\frac{n}{2}} V\left(\xi z^{-\frac{1}{2}}\right)\right) d z\right\|_{\mathbf{Q}} \\
& \leqslant C|\theta|+\left\|t^{\frac{n}{2}} \int_{0}^{t} \mathscr{G}_{0}\left(t-\tau, \xi t^{1 / 2}\right) \mathscr{N}(v(\tau)) d \tau\right\|_{\mathbf{Q}} \\
& =C|\theta|+\left\|\int_{0}^{t} \mathscr{G}_{0}(t-\tau) \mathscr{N}(v(\tau)) d \tau\right\|_{\mathbf{X}} .
\end{aligned}
$$

Из (65) получаем

$$
\left\|\int_{0}^{t} \mathscr{G}_{0}(t-\tau) \mathscr{N}(v(\tau)) d \tau\right\|_{\mathbf{X}} \leqslant C\|v\|_{\mathbf{X}}^{\sigma+1}=C\|V\|_{\mathbf{Q}}^{\sigma+1} \leqslant C \rho^{\sigma+1} .
$$

Поэтому $\|\mathscr{R}(V)\|_{\mathbf{Q}} \leqslant C|\theta|+C \rho^{\sigma+1} \leqslant C \rho$, так как $\rho>0$ мало. Точно так же оценивается разность $\mathscr{R}(V)-\mathscr{R}(W)$ : ввиду связи норм пространств $\mathbf{Q}$ и $\mathbf{X}$ 
находим

$$
\begin{aligned}
\|\mathscr{R}(V)-\mathscr{R}(W)\|_{\mathbf{Q}} & \leqslant\left\|\int_{0}^{1} \mathscr{G}_{0}(1-z, \xi)\left(\mathscr{N}\left(z^{-\frac{n}{2}} V\left(\xi z^{-\frac{1}{2}}\right)\right)-\mathscr{N}\left(z^{-\frac{n}{2}} W\left(\xi z^{-\frac{1}{2}}\right)\right)\right) d z\right\|_{\mathbf{Q}} \\
& =\left\|\int_{0}^{t} \mathscr{G}_{0}(t-\tau)(\mathscr{N}(v(\tau))-\mathscr{N}(w(\tau))) d \tau\right\|_{\mathbf{X}},
\end{aligned}
$$

где $w(t, x)=t^{-n / 2} W\left(x t^{-1 / 2}\right)$, откуда в силу (65)

$$
\begin{aligned}
\|\mathscr{R}(V)-\mathscr{R}(W)\|_{\mathbf{Q}} & \leqslant C\|V-W\|_{\mathbf{Q}}\left(\|V\|_{\mathbf{Q}}^{\sigma}+\|W\|_{\mathbf{Q}}^{\sigma}\right) \\
& \leqslant C \rho^{\sigma}\|V-W\|_{\mathbf{Q}} \leqslant \frac{1}{2}\|V-W\|_{\mathbf{Q}} .
\end{aligned}
$$

Таким образом, $\mathscr{R}$ является сжимающим отображением в $\mathbf{Q}_{\rho}$ и, значит, существует единственное решение $V \in \mathbf{Q}_{\rho}$ интегрального уравнения (66). Лемма 3 доказана.

Теперь покажем, что автомодельное решение $V(\xi)$ дает асимптотику решений задачи Коши (62). Обозначим $\theta=f\left(u_{0}\right)$.

Теорема 13. Пусть начальные возмущения $u_{0} \in \mathbf{Z}$ имеют малую норму $\left\|u_{0}\right\|_{\mathbf{Z}} \leqslant \varepsilon$ с достаточно малым $\varepsilon>0$. Предположим, что нелинейность $\mathscr{N}$ в уравнении (62) является критической конвективной. Пусть выполнены оценки

$$
\begin{aligned}
& \left\|\langle t\rangle^{\gamma} \int_{0}^{t} \mathscr{G}(t-\tau)(\mathscr{N}(v(\tau))-\mathscr{N}(w(\tau))) d \tau\right\|_{\mathbf{X}} \\
& \quad \leqslant C\left\|\langle t\rangle^{\gamma}(v(t)-w(t))\right\|_{\mathbf{X}}\left(\|v\|_{\mathbf{X}}+\|w\|_{\mathbf{X}}\right)^{\sigma}
\end{aligned}
$$

$u$

$$
\left\|\langle t\rangle^{\gamma} \int_{0}^{t}\left(\mathscr{G}(t-\tau)-\mathscr{G}_{0}(t-\tau)\right) \mathscr{N}(v(\tau)) d \tau\right\|_{\mathbf{X}} \leqslant C\|v\|_{\mathbf{X}}^{1+\sigma}
$$

для всех $v, w \in \mathbf{X}$, где $\sigma>0, \gamma>0$, а $\mathscr{G}_{0}$ является автомодельным асимптотическим оператором для оператора Грина $\mathscr{G}$ в пространствах $\mathbf{X}, \mathbf{Z}$. Предположим, что существует единственное глобальное во времени решение $u \in \mathbf{X}$ задачи Коши (62) с малой нормой $\|u\|_{\mathbf{X}} \leqslant C \varepsilon$. Тогда для этого решения имеет место следующая асимптотическал оченка:

$$
\left\|\langle t\rangle^{\gamma}\left(u(t)-t^{-\frac{n}{2}} V\left(x t^{-\frac{1}{2}}\right)\right)\right\|_{\mathbf{X}} \leqslant C
$$

при $t \rightarrow \infty$, где V является решением интегрального уравнения (66) $c \theta=f\left(u_{0}\right)$.

ДокАЗАтЕльство. Обозначим $v(t, x)=t^{-n / 2} \theta V\left(x t^{-1 / 2}\right)$, где $V$ удовлетворяет интегральному уравнению (66) с $\theta=f\left(u_{0}\right)$. Тогда

$$
v(t)=\theta G_{0}(t)-\int_{0}^{t} \mathscr{G}_{0}(t-\tau) \mathscr{N}(v(\tau)) d \tau,
$$

где $G_{0}$ имеет автомодельный вид: $G_{0}(t, x)=t^{-n / 2} \widetilde{G}_{0}\left(x t^{-1 / 2}\right)$. Поскольку $u(t)$ удовлетворяет интегральному уравнению

$$
u(t)=\mathscr{G}(t) u_{0}-\int_{0}^{t} \mathscr{G}(t-\tau) \mathscr{N}(u(\tau)) d \tau,
$$


то выполнена оценка

$$
\begin{aligned}
\|\langle t\rangle^{\gamma}(u(t) & -v(t))\left\|_{\mathbf{X}} \leqslant\right\|\langle t\rangle^{\gamma}\left(\mathscr{G}(t) u_{0}-\theta G_{0}(t)\right) \|_{\mathbf{X}} \\
& +\left\|\langle t\rangle^{\gamma} \int_{0}^{t} \mathscr{G}(t-\tau)(\mathscr{N}(u(\tau))-\mathscr{N}(v(\tau))) d \tau\right\|_{\mathbf{X}} \\
& +\left\|\langle t\rangle^{\gamma} \int_{0}^{t}\left(\mathscr{G}(t-\tau)-\mathscr{G}_{0}(t-\tau)\right) \mathscr{N}(v(\tau)) d \tau\right\|_{\mathbf{X}} \equiv I_{1}+I_{2}+I_{3} .
\end{aligned}
$$

Отметим, что ввиду связи норм пространств $\mathbf{X}$ и $\mathbf{Q}$ справедливо неравенство $\|v\|_{\mathbf{x}}=|\theta|\|V\|_{\mathbf{Q}} \leqslant C|\theta| \leqslant C \varepsilon$. Тогда по определению асимптотического ядра (см. (18)) получим $I_{1} \leqslant C\left\|u_{0}\right\|_{\mathbf{z}} \leqslant C \varepsilon$. В силу условия (67) имеем

$$
\begin{aligned}
I_{2} & \leqslant C\left\|\langle t\rangle^{\gamma}(u(t)-v(t))\right\|_{\mathbf{X}}\left(\|u\|_{\mathbf{X}}^{\sigma}+\|v\|_{\mathbf{X}}^{\sigma}\right) \\
& \leqslant C \varepsilon^{\sigma}\left\|\langle t\rangle^{\gamma}(u(t)-v(t))\right\|_{\mathbf{X}}
\end{aligned}
$$

и благодаря условию (68) найдем

$$
I_{3} \leqslant C\|v\|_{\mathbf{X}}^{\sigma+1} \leqslant C\|V\|_{\mathbf{Q}}^{\sigma+1} \leqslant C|\theta|^{\sigma+1} \leqslant C \varepsilon^{\sigma+1}
$$

Теперь (70) влечет $\left\|\langle t\rangle^{\gamma}(u(t)-v(t))\right\|_{\mathbf{X}} \leqslant C \varepsilon+C \varepsilon^{\sigma}\left\|\langle t\rangle^{\gamma}(u(t)-v(t))\right\|_{\mathbf{X}}$, так что справедлива оценка (69), поскольку $\varepsilon>0$ достаточно мало. Теорема 13 доказана.

Применим теорему 13 к уравнению Соболева (24) с критическим показателем $\sigma=1 / n$. Определим пространство $\mathbf{Z}=\mathbf{L}^{1, a}\left(\mathbb{R}^{n}\right) \cap \mathbf{W}_{\infty}^{1}\left(\mathbb{R}^{n}\right)$ при $a \in(0,1)$ и введем пространство

$$
\mathbf{X}=\left\{\phi \in \mathbf{C}\left([0, \infty) ; \mathbf{L}^{1, a}\left(\mathbb{R}^{n}\right) \cap \mathbf{W}_{\infty}^{1}\left(\mathbb{R}^{n}\right)\right):\|\phi\|_{\mathbf{X}}<\infty\right\}
$$

с нормой

$$
\|\phi\|_{\mathbf{X}}=\sup _{t>0}\left(\|\phi(t)\|_{\mathbf{L}^{1}}+\langle t\rangle^{\frac{n}{2}}\|\phi(t)\|_{\mathbf{L}^{\infty}}+\langle t\rangle^{\frac{n+1}{2}}\|\nabla \phi(t)\|_{\mathbf{L}^{\infty}}\right)
$$

Также зададим норму $\|\phi\|_{\mathbf{Q}}=\|\phi\|_{\mathbf{L}^{1}}+\|\phi\|_{\mathbf{L}^{\infty}}+\|\nabla \phi\|_{\mathbf{L}^{\infty}}$, так что имеет место соотношение $\left\|t^{-n / 2} \phi\left(x t^{-1 / 2}\right)\right\|_{\mathbf{x}}=\|\phi\|_{\mathbf{Q}}$. Заметим, что оператор

$$
\mathscr{G}_{0}(t) \phi=\int_{\mathbb{R}^{n}} G_{0}(t, x-y) \phi(y) d y
$$

с ядром $G_{0}(t, x)=(4 \pi \alpha t)^{-n / 2} e^{-|x|^{2} /(4 \alpha t)}$ является автомодельным асимптотическим в пространствах $\mathbf{X}$ и $\mathbf{Z}$ для оператора $\mathscr{G}_{2}(t)$ при выборе функционала $f$ в виде интеграла $\int_{\mathbb{R}^{n}} \phi(x) d x$.

Нелинейность $\mathscr{N}(u)=(\lambda \cdot \nabla)|u|^{\sigma} u$ в уравнении (24) имеет форму полной производной, так что выполнено свойство конвективности $f(\mathscr{N}(u))=0$. Проверим, что при $\sigma=1 / n$ нелинейность является критической. Сделав замену 
переменных $x=\xi t^{1 / 2}, \tau=z t, y=y^{\prime} t^{1 / 2}$, найдем

$$
\begin{aligned}
\int_{0}^{t} \mathscr{G}_{0}(t-\tau, x) \mathscr{N}\left(\tau^{-\frac{n}{2}} V\left(x \tau^{-\frac{1}{2}}\right)\right) d \tau \\
\quad=\int_{0}^{t}(t-\tau)^{-\frac{n}{2}} \int_{\mathbb{R}^{n}} \widetilde{G}_{0}\left((x-y)(t-\tau)^{-\frac{1}{2}}\right) \mathscr{N}\left(\tau^{-\frac{n}{2}} V\left(y \tau^{-\frac{1}{2}}\right)\right) d y d \tau \\
\quad=t^{-\frac{n}{2}} \int_{0}^{1} d z(1-z)^{-\frac{n}{2}} \int_{\mathbb{R}^{n}} \widetilde{G}_{0}\left(\left(\xi-y^{\prime}\right)(1-z)^{-\frac{1}{2}}\right) \mathscr{N}\left(z^{-\frac{n}{2}} V\left(y^{\prime} z^{-\frac{1}{2}}\right)\right) d y^{\prime} \\
=t^{-\frac{1}{2}} \int_{0}^{1} \mathscr{G}_{0}(1-z, \xi) \mathscr{N}\left(z^{-\frac{n}{2}} V\left(x z^{-\frac{1}{2}}\right)\right) d z
\end{aligned}
$$

Таким образом, мы видим, что условие (64) выполнено. Значит, нелинейность $\mathscr{N}$ является критической конвективной.

Теорема 14. Пусть $\sigma=1 / n, n=1,2,3$. Предположим, что

$$
u_{0} \in \mathbf{L}^{1, a}\left(\mathbb{R}^{n}\right) \cap \mathbf{W}_{\infty}^{1}\left(\mathbb{R}^{n}\right), \quad a \in(0,1),
$$

причем норма $\left\|u_{0}\right\|_{\mathbf{L}^{1, a}}+\left\|u_{0}\right\|_{\mathbf{W}_{\infty}^{1}}$ достаточно мала. Тогда существует единственное решение $u \in \mathbf{C}\left([0, \infty) ; \mathbf{L}^{1, a}\left(\mathbb{R}^{n}\right) \cap \mathbf{W}_{\infty}^{1}\left(\mathbb{R}^{n}\right)\right)$ задачи Коши (24). Для этого решения справедлива асимптотика $u(t, x)=t^{-n / 2} V\left(x t^{-1 / 2}\right)+O\left(t^{-n / 2-\gamma}\right)$ при $t \rightarrow \infty$ равномерно по $x \in \mathbb{R}^{n}$, где $0<\gamma<\min (a / 2, n \sigma / 2-1)$ u $V \in \mathbf{L}^{\infty}\left(\mathbb{R}^{n}\right)$ является решением интегрального уравнения

$$
V(\xi)=\theta \widetilde{G}_{0}(\xi)-\int_{0}^{1} \frac{d z}{z(1-z)^{\frac{n}{2}}} \int_{\mathbb{R}^{n}} \widetilde{G}_{0}\left(\frac{\xi-y z^{1 / 2}}{\sqrt{1-z}}\right) \mathscr{N}(V(y)) d y .
$$

Здесъ $\widetilde{G}_{0}(\xi)=(4 \pi \alpha)^{-n / 2} e^{-|\xi|^{2} /(4 \alpha)} u \theta=\int_{\mathbb{R}^{n}} u_{0}(x) d x$.

ДокАзАтельство. Докажем сначала оценку

$$
\left\|\langle t\rangle^{\gamma} \int_{0}^{t} \mathscr{G}_{2}(t-\tau)(\lambda \cdot \nabla) \phi(\tau) d \tau\right\|_{\mathbf{X}} \leqslant C\left\|\sqrt{t}\langle t\rangle^{\gamma} \phi(t)\right\|_{\mathbf{X}}
$$

где $0 \leqslant \gamma<1 / 2$. Поскольку $f(\phi)=0$, в силу леммы 2 получаем оценки

$$
\begin{aligned}
& \left\|\int_{0}^{t} \mathscr{G}_{2}(t-\tau)(\lambda \cdot \nabla) \phi(\tau) d \tau\right\|_{\mathbf{L}^{1}} \leqslant \int_{0}^{\frac{t}{2}}(t-\tau)^{-\frac{1}{2}} \tau^{-\frac{1}{2}}\langle\tau\rangle^{-\gamma} d \tau \sup _{\tau>0} \sqrt{\tau}\langle\tau\rangle^{\gamma}\|\phi(\tau)\|_{\mathbf{L}^{1}} \\
& \quad+\int_{\frac{t}{2}}^{t} \tau^{-1}\langle\tau\rangle^{-\gamma} d \tau \sup _{\tau>0} \tau\langle\tau\rangle^{\gamma}\|\nabla \phi(\tau)\|_{\mathbf{L}^{1}} \leqslant C\langle t\rangle^{-\gamma}\left\|\sqrt{t}\langle t\rangle^{\gamma} \phi(t)\right\|_{\mathbf{X}}, \\
& \left\|\int_{0}^{t} \mathscr{G}(t-\tau)(\lambda \cdot \nabla) \phi(\tau) d \tau\right\|_{\mathbf{L}^{\infty}} \leqslant \int_{0}^{\frac{t}{2}}(t-\tau)^{-\frac{n+1}{2}} \tau^{-\frac{1}{2}}\langle\tau\rangle^{-\gamma} d \tau \sup _{\tau>0} \sqrt{\tau}\langle\tau\rangle^{\gamma}\|\phi(\tau)\|_{\mathbf{L}^{1}} \\
& \quad+\int_{\frac{t}{2}}^{t} \tau^{-1-\frac{n}{2}}\langle\tau\rangle^{-\gamma} d \tau \sup _{\tau>0} \tau^{1+\frac{n}{2}}\langle\tau\rangle^{\gamma}\|\nabla \phi(\tau)\|_{\mathbf{L}^{\infty}} \leqslant C t^{-\frac{n}{2}}\langle t\rangle^{-\gamma}\left\|\sqrt{t}\langle t\rangle^{\gamma} \phi(t)\right\|_{\mathbf{X}},
\end{aligned}
$$




$$
\begin{aligned}
\| \nabla & \int_{0}^{t} \mathscr{G}(t-\tau)(\lambda \cdot \nabla) \phi(\tau) d \tau \|_{\mathbf{L}^{\infty}} \\
\leqslant & \int_{0}^{\frac{t}{2}}(t-\tau)^{-\frac{n+2}{2}} \tau^{-\frac{1}{2}}\langle\tau\rangle^{-\gamma} d \tau \sup _{\tau>0} \sqrt{\tau}\langle\tau\rangle^{\gamma}\|\phi(\tau)\|_{\mathbf{L}^{1}} \\
& \quad+\int_{\frac{t}{2}}^{t}(t-\tau)^{-\frac{1}{2}} \tau^{-1-\frac{n}{2}}\langle\tau\rangle^{-\gamma} d \tau \sup _{\tau>0} \tau^{1+\frac{n}{2}}\langle\tau\rangle^{\gamma}\|\nabla \phi(\tau)\|_{\mathbf{L}^{\infty}} \\
\leqslant & C t^{-\frac{n+1}{2}}\langle t\rangle^{-\gamma}\left\|\sqrt{t}\langle t\rangle^{\gamma} \phi(t)\right\|_{\mathbf{X}}
\end{aligned}
$$

для всех $t>0$, откуда следует (71).

По определению нормы пространства $\mathbf{X}$ получим при $\sigma=1 / n$

$$
\left\|\sqrt{t}\langle t\rangle^{\gamma}\left(|v|^{\sigma} v(t)-|w|^{\sigma} w(t)\right)\right\|_{\mathbf{X}} \leqslant C\left\|\langle t\rangle^{\gamma}(v(t)-w(t))\right\|_{\mathbf{X}}\left(\|v\|_{\mathbf{X}}+\|w\|_{\mathbf{X}}\right)^{\sigma} .
$$

Поэтому, взяв в оценке $(71) \phi=|v|^{\sigma} v-|w|^{\sigma} w$, получим неравенства (67) и (68). Существование глобального решения $u \in \mathbf{X}$ устанавливается так же, как и выше в доказательстве теоремы 4. Таким образом, все условия теоремы 13 выполнены. Применяя теорему 13, приходим к результату теоремы 14 .

5.2. Большие начальные данные. Рассмотрим уравнение (24) в случае не малых начальных данных. Рассмотрим для простоты случай $\sigma=1, n=1$. Докажем следующий результат.

Теорема 15. Пусть $\sigma=1, \lambda \in \mathbb{R}, n=1$. Предположим, ито $u_{0} \in \mathbf{H}^{2}(\mathbb{R}) \cap$ $\mathbf{W}_{1}^{2}(\mathbb{R}) \cap \mathbf{L}^{1, a}(\mathbb{R}), a \geqslant 0, u \theta=\int_{\mathbb{R}} u_{0}(x) d x \neq 0$. Тогда существует единственное решение $и \in \mathbf{C}\left([0, \infty) ; \mathbf{H}^{2}(\mathbb{R}) \cap \mathbf{W}_{1}^{2}(\mathbb{R}) \cap \mathbf{L}^{1, a}(\mathbb{R})\right)$ задачи Коши (24). Для этого решения справедлива асимптотика $u(t)=t^{-1 / 2} f_{\theta}\left(x t^{-1 / 2}\right)+o\left(t^{-1 / 2}\right)$ при $t \rightarrow \infty$ равномерно по $x \in \mathbb{R}$. Если дополнительно $u_{0} \in \mathbf{L}^{1,1}(\mathbb{R})$, то имеет место асимптотика

$$
u(t)=t^{-1 / 2} f_{\theta}\left(x t^{-1 / 2}\right)+O\left(t^{-1 / 2-\gamma}\right)
$$

при $t \rightarrow \infty$, где $\gamma \in(0,1 / 2)$ и функиия

$$
f_{\theta}(\chi)=-2 \sqrt{\mu} \frac{\partial}{\partial \chi} \log \left(\cosh \frac{\theta}{4}-\sinh \left(\frac{\theta}{4}\right) \operatorname{Erf}\left(\frac{\chi}{2 \sqrt{\mu}}\right)\right)
$$

является автомодельным решением уравнения Бюргерса $u_{t}+u u_{x}-\mu u_{x x}=0$, определяемым общей массой $\theta=\int_{\mathbb{R}} u_{0}(x) d x$ начальных данных, здесъ $\operatorname{Erf}(x)=$ $\frac{2}{\sqrt{\pi}} \int_{0}^{x} e^{-y^{2}} d y-$ интеграл ошибок.

ДокАЗАТЕЛЬСтво. Возьмем достаточно большое время $T>0$ и определим $v(t, x)$ как решение уравнения Бюргерса с начальным возмущением $u(T, x)$

$$
\begin{gathered}
v_{t}+v v_{x}-\alpha v_{x x}=0, \quad x \in \mathbb{R}, \quad t>T, \\
v(T, x)=u(T, x), \quad x \in \mathbb{R} .
\end{gathered}
$$


С помощью замены Хопфа-Коула $v(t, x)=-2 \alpha \frac{\partial}{\partial x} \log Z(t, x)$ преобразуем это уравнение к уравнению теплопроводности $Z_{t}=\alpha Z_{x x}$, которое допускает решение в явном виде:

$$
Z(t, x)=\int_{\mathbb{R}} d y G_{0}(t, x-y) \exp \left\{-\frac{1}{2 \alpha} \int_{-\infty}^{y} u(T, \xi) d \xi\right\},
$$

где $G_{0}(t, x)=(4 \pi \alpha t)^{-1 / 2} e^{-x^{2} /(4 \alpha t)}$. Имеет место оценка

$$
\|v(t)\|_{\mathbf{L}^{p}}+\langle t\rangle\left\|\partial_{x}^{2} \mathscr{B}_{2} v(t)\right\|_{\mathbf{L}^{p}} \leqslant C\langle t\rangle^{-\frac{1}{2}\left(1-\frac{1}{p}\right)}
$$

для всех $t>T, 1 \leqslant p \leqslant \infty$. Для разности $w(t, x)=u(t, x)-v(t, x)$ получим задачу

$$
\begin{gathered}
w_{t}+\partial_{x}(v w)+\frac{1}{2} \frac{\partial}{\partial x} w^{2}-\alpha w_{x x}+h_{x}=0, \quad x \in \mathbb{R}, \quad t>T, \\
w(T, x)=0, \quad x \in \mathbb{R},
\end{gathered}
$$

где $h=\left(\beta \partial_{x}^{2}+\alpha \partial_{x}^{3}\right) \mathscr{B}_{2} u-\partial_{x} \mathscr{B}_{2}\left(u u_{x}\right)$. В случае не малых начальных возмущений необходимо устранить слагаемое $\frac{\partial}{\partial x}(v w)$. Для этого сделаем замену $\partial_{x}^{-1} w=$ $\int_{-\infty}^{x} w(t, y) d y=\alpha g / Z$, тогда

$$
\begin{aligned}
g_{t}-\alpha g_{x x}+F=0, & x \in \mathbb{R}, \quad t>T, \\
g(T, x)=0, \quad x \in \mathbb{R}, &
\end{aligned}
$$

где

$$
F=\frac{\mu}{2 Z}\left(g_{x}+\frac{1}{2 \alpha} g v\right)^{2}+Z h .
$$

Имеем $\|Z(t)\|_{\mathbf{L}^{\infty}}+\left\|Z^{-1}(t)\right\|_{\mathbf{L}^{\infty}} \leqslant C$ при всех $t \geqslant T$, а также грубую оценку убывания

$$
\|w(t)\|_{\mathbf{L}^{p}}+\langle t\rangle\left\|\partial_{x}^{2} \mathscr{B}_{2} w(t)\right\|_{\mathbf{L}^{p}} \leqslant C\langle t\rangle^{-(1-1 / p) / 2}
$$

при всех $t \geqslant T, 1 \leqslant p \leqslant \infty$. Докажем неравенство

$$
\|g(t)\|_{\mathbf{L}^{p}}+\langle t\rangle^{1 / 2}\left\|g_{x}(t)\right\|_{\mathbf{L}^{p}}<C\langle t\rangle^{-\gamma+\frac{1}{2 p}}
$$

при всех $t \geqslant T, 2 \leqslant p \leqslant \infty$ с некоторым $\gamma \in(0,1 / 2)$. От противного, пусть в некоторый момент времени $t=T_{1}$ оценка (72) нарушается, так что по непрерывности имеем

$$
\|g(t)\|_{\mathbf{L}^{p}}+\langle t\rangle^{1 / 2}\left\|g_{x}(t)\right\|_{\mathbf{L}^{p}} \leqslant C\langle t\rangle^{-\gamma+\frac{1}{2 p}}
$$

для всех $t \in\left[T, T_{1}\right], 1 \leqslant p \leqslant \infty$. Ввиду (73) найдем

$$
\begin{aligned}
\|F(t)\|_{\mathbf{L}^{p}} & \leqslant C\left\|g_{x}\right\|_{\mathbf{L}^{\infty}}\left\|g_{x}\right\|_{\mathbf{L}^{p}}+C\|g\|_{\mathbf{L}^{\infty}}^{2}\left\|v^{2}\right\|_{\mathbf{L}^{p}}+C\|Z h\|_{\mathbf{L}^{p}} \\
& \leqslant C\left(\langle t\rangle^{-1-2 \gamma+\frac{1}{2 p}}+\langle t\rangle^{-\frac{3}{2}+\frac{1}{2 p}}\right) \leqslant C \max \left(T^{\gamma-\frac{1}{2}}, T^{-\gamma}\right)\langle t\rangle^{-\gamma-1+\frac{1}{2 p}}
\end{aligned}
$$


для всех $t \in\left[T, T_{1}\right], 1 \leqslant p \leqslant \infty$. Тогда из соответствующего интегрального уравнения получаем

$$
\begin{aligned}
\|g(t)\|_{\mathbf{L}^{p}} & \leqslant \int_{T}^{t} d \tau\left\|G_{0}(t-\tau)\right\|_{\mathbf{L}^{p}}\|F(\tau)\|_{\mathbf{L}^{1}} \\
& \leqslant C \max \left(T^{\gamma-\frac{1}{2}}, T^{-\gamma}\right) \int_{T}^{t}(t-\tau)^{-\frac{1}{2}+\frac{1}{2 p}}\langle\tau\rangle^{-\frac{1}{2}-\gamma} d \tau \\
& \leqslant C \max \left(T^{\gamma-\frac{1}{2}}, T^{-\gamma}\right)\langle t\rangle^{-\gamma+\frac{1}{2 p}}<C\langle t\rangle^{-\gamma+\frac{1}{2 p}}
\end{aligned}
$$

для всех $t \in\left[T, T_{1}\right], 1 \leqslant p \leqslant \infty$, поскольку $T$ достаточно велико. Аналогично,

$$
\begin{aligned}
\left\|g_{x}(t)\right\|_{\mathbf{L}^{p}} \leqslant & \int_{T}^{\frac{t+T}{2}} d \tau\left\|\partial_{x} G_{0}(t-\tau)\right\|_{\mathbf{L}^{p}}\|F(\tau)\|_{\mathbf{L}^{1}} \\
& \quad+\int_{\frac{t+T}{2}}^{t} d \tau\left\|\partial_{x} G_{0}(t-\tau)\right\|_{\mathbf{L}^{1}}\|F(\tau)\|_{\mathbf{L}^{p}} \\
\leqslant & C \max \left(T^{\gamma-\frac{1}{2}}, T^{-\gamma}\right) \int_{T}^{\frac{t+T}{2}}(t-\tau)^{-1+\frac{1}{2 p}}\langle\tau\rangle^{-\frac{1}{2}-\gamma} d \tau \\
& +C \max \left(T^{\gamma-\frac{1}{2}}, T^{-\gamma}\right) \int_{\frac{t+T}{2}}^{t}(t-\tau)^{-\frac{1}{2}}\langle\tau\rangle^{-1-\gamma+\frac{1}{2 p}} d \tau \\
\leqslant & C \max \left(T^{\gamma-\frac{1}{2}}, T^{-\gamma}\right)\langle t\rangle^{-\gamma-\frac{1}{2}+\frac{1}{2 p}}<C\langle t\rangle^{-\gamma-\frac{1}{2}+\frac{1}{2 p}}
\end{aligned}
$$

для всех $t \in\left[T, T_{1}\right], 1 \leqslant p \leqslant \infty$. Полученное противоречие доказывает (72) для всех $t \geqslant T$. Так как $w=Z^{-1}\left(\mu g_{x}+g v / 2\right)$, то оценка $(72)$ дает $\|u(t)-v(t)\|_{\mathbf{L}^{\infty}} \leqslant$ $C\langle t\rangle^{-\gamma-1 / 2}$ для всех $t>T$. Если дополнительно известно, что $x u_{0} \in \mathbf{L}^{1}(\mathbb{R})$, то имеем $\left.\left\|v(t)-t^{-1 / 2} f_{\theta}\left(x t^{-1 / 2}\right)\right\|_{\mathbf{L}} \infty \leqslant C t\right\rangle^{-\gamma-1 / 2}$. Откуда приходим к асимптотике теоремы. Теорема 15 доказана.

\section{6. Субкритическая неконвективная нелинейность}

6.1. Малые начальные данные. В этом разделе мы изучим асимптотику при больших временах решений уравнений типа Соболева с субкритической нелинейностью неконвективного типа. Мы покажем, что нелинейность существенно влияет на асимптотику решения и характер асимптотики определяется автомодельными решениями. Рассмотрим задачу Коши

$$
\begin{gathered}
u_{t}+\mathscr{N}(u)+\mathscr{L} u=0, \quad x \in \mathbb{R}^{n}, \quad t>0, \\
u(0, x)=u_{0}(x), \quad x \in \mathbb{R}^{n},
\end{gathered}
$$

с субкритической нелинейностью $\mathscr{N}(u)$ неконвективного типа (см. (75)). Пусть $\mathbf{Z}$ - некоторое метрическое пространство функций на $\mathbb{R}^{n}$, также фиксируем полное метрическое пространство $\mathbf{X}$ функций на $[0, \infty) \times \mathbb{R}^{n}$. Как и выше, будем обозначать через $G_{0} \in \mathbf{X}$ асимптотическое ядро оператора Грина $\mathscr{G}$ в пространствах $\mathbf{X}$ и $\mathbf{Z}$ с соответствующим непрерывным линейным функционалом $f$ (см. определение (18) выше). Будем называть нелинейность $\mathscr{N}$ субкритической неконвективной, если выполнена оценка

$$
\frac{\sigma}{\theta} \operatorname{Re} f\left(\mathscr{N}\left(\theta G_{0}(t)\right)\right) \geqslant \frac{\eta}{2} \theta^{\sigma} t^{\mu-1}
$$


для всех $t>0, \theta>0$ с некоторыми положительными постоянными $\eta, \sigma$ и $\mu \in$ $(0,1)$. Смысл субкритичности заключается в достаточно медленном убывании по времени.

Докажем сначала существование глобальных решений задачи Коши (74).

Теорема 16. Предположим, что линейный оператор $\mathscr{L}$ удовлетворяет условию $f(\mathscr{L} u)=0$ для любой $u \in \mathbf{X}$. Пусть нелинейность $\mathscr{N}(u)$ в уравнении (74) является субкритической неконвективной. Предположим, что

$$
e^{z} \mathscr{N}\left(u e^{-z}\right)=e^{-\sigma \operatorname{Re} z} \mathscr{N}(u)
$$

для всех $z \in \mathbb{C} u u \in \mathbf{X}$ с некоторым $\sigma>0$. Пусть для всех $t>0$ выполнена оценка

$$
\begin{aligned}
& \nu(t)|f(\mathscr{N}(v(t))-\mathscr{N}(w(t)))| \\
& \quad \leqslant C\{t\}^{-\alpha}\langle t\rangle^{\mu-1}\|\nu(t)(v(t)-w(t))\|_{\mathbf{X}}\left(\|v\|_{\mathbf{X}}^{\sigma}+\|w\|_{\mathbf{X}}^{\sigma}\right)
\end{aligned}
$$

для любых $v, w \in \mathbf{X}$, где $\nu(t)>0, \alpha<1, \sigma>0, \mu \in(0,1)$, а также оценка

$$
\begin{aligned}
& \left\|\int_{0}^{t}|\mathscr{G}(t-\tau)(\mathscr{K}(v(\tau))-\mathscr{K}(w(\tau)))| \tau^{-\mu} d \tau\right\|_{\mathbf{X}} \\
& \quad \leqslant C\|v-w\|_{\mathbf{X}}\left(\|v\|_{\mathbf{X}}+\|w\|_{\mathbf{X}}\right)^{\sigma}\left(1+\frac{1}{\theta}\left(\|v\|_{\mathbf{X}}+\|w\|_{\mathbf{X}}\right)\right)
\end{aligned}
$$

для любых $v, w \in \mathbf{X}$, удовлетворяющих условию $f(v)=f(w)=\theta>0$, где $\sigma>0$, $\mathscr{K}(v)=\mathscr{N}(v)-(v / \theta) f(\mathscr{N}(v))$. Пусть начальные возмущения $u_{0} \in \mathbf{Z}$ маль: $\left\|u_{0}\right\|_{\mathbf{z}} \leqslant \varepsilon, a \theta \equiv\left|f\left(u_{0}\right)\right| \geqslant C \varepsilon>0$ с некоторым $C>0$. Также пусть $\mu \in(0,1)$ в (75) и (77) достаточно мало. Тогда существует единственное глобальное во времени решение $u \in \mathbf{X}$ задачи Коши (74). Для этого решения справедлива оценка $\left\|t^{\mu / \sigma} u(t)\right\|_{\mathbf{X}} \leqslant C \varepsilon$.

ДокАЗАТЕЛЬство. Как и при доказательстве теоремы 9, сделаем замену $u(t, x)=v(t, x) e^{-\varphi(t)+i \psi(t)}$ в уравнении $(74)$, тогда получим интегральное уравнение

$$
v(t)=\mathscr{G}(t) v_{0}-\int_{0}^{t} \mathscr{G}(t-\tau) \mathscr{K}(v(\tau)) \frac{d \tau}{h_{v}(\tau)}
$$

с новой нелинейностью

$$
\mathscr{K}(v(\tau))=\mathscr{N}(v(\tau))-\frac{v(\tau)}{\theta} f(\mathscr{N}(v(\tau)))
$$

и функционалом

$$
h_{v}(t)=1+\frac{\sigma}{\theta} \int_{0}^{t} \operatorname{Re} f(\mathscr{N}(v(\tau))) d \tau
$$

Заметим, что функция $\psi(t)$ является решением задачи Коши

$$
\psi^{\prime}(t)=-\frac{1}{\theta h_{v}(t)} \operatorname{Im} f(\mathscr{N}(v(t))), \quad \psi(0)=\arg f\left(u_{0}\right) .
$$


Для того чтобы применить принцип сжимающих отображений, зададим преобразование

$$
\mathscr{M}(w)=\mathscr{G}(t) v_{0}-\int_{0}^{t} \mathscr{G}(t-\tau) \mathscr{K}(w(\tau)) \frac{d \tau}{h_{w}(\tau)}
$$

для любой функции $w$ из множества

$$
\begin{aligned}
& \mathbf{B}=\{w \in \mathbf{X}: f(w)=\theta,\|w\|_{\mathbf{x}} \leqslant C \varepsilon \\
&\left.\left\|w-\mathscr{G}(t) v_{0}\right\|_{\mathbf{X}} \leqslant C \mu \varepsilon, \sup _{t>0} t^{-\mu} h_{w}(t) \geqslant \frac{\eta}{3 \mu} \theta^{\sigma}\right\},
\end{aligned}
$$

где

$$
h_{w}(t)=1+\frac{\sigma}{\theta} \int_{0}^{t} \operatorname{Re} f(\mathscr{N}(w(\tau))) d \tau .
$$

Проверим сначала, что $\mathscr{M}$ отображает В в себя. Поскольку $f(w)=\theta$, имеем $f(\mathscr{K}(w))=f(\mathscr{N}(w))-\theta^{-1} f(\mathscr{N}(w)) f(w)=0$; откуда ввиду определения $\mathscr{M}(w)$ получим равенства

$$
f(\mathscr{M}(w))=f\left(\mathscr{G}(t) v_{0}\right)=f\left(v_{0}\right)=\theta .
$$

По определению асимптотического ядра $\left\|\langle t\rangle^{\gamma}\left(\mathscr{G}(t) v_{0}-\theta G_{0}(t)\right)\right\|_{\mathbf{X}} \leqslant C\left\|v_{0}\right\|_{\mathbf{Z}} \leqslant$ $C \varepsilon$, и поскольку $w \in \mathbf{B}$, то по условию (78)

$$
\begin{aligned}
\left\|\int_{0}^{t} \mathscr{G}(t-\tau) \mathscr{K}(w(\tau)) \frac{d \tau}{h_{w}(\tau)}\right\|_{\mathbf{X}} & \leqslant \frac{3 \mu}{\eta} \theta^{-\sigma}\left\|\int_{0}^{t} \tau^{-\mu}|\mathscr{G}(t-\tau) \mathscr{K}(w(\tau))| d \tau\right\|_{\mathbf{X}} \\
& \leqslant \frac{C \mu}{\theta^{\sigma}}\|w\|_{\mathbf{X}}^{\sigma+1}\left(1+\frac{\|w\|_{\mathbf{X}}}{\theta}\right) \leqslant C \mu \varepsilon .
\end{aligned}
$$

Поэтому

$$
\left\|\mathscr{M}(w)-\mathscr{G}(t) v_{0}\right\|_{\mathbf{X}} \leqslant\left\|\int_{0}^{t} \mathscr{G}(t-\tau) \mathscr{K}(w(\tau)) \frac{d \tau}{h_{w}(\tau)}\right\|_{\mathbf{X}} \leqslant C \mu \varepsilon
$$

и

$$
\|\mathscr{M}(w)\|_{\mathbf{x}} \leqslant\left\|\mathscr{G}(t) v_{0}\right\|_{\mathbf{x}}+\left\|\mathscr{M}(w)-\mathscr{G}(t) v_{0}\right\|_{\mathbf{x}} \leqslant C \varepsilon+C \mu \varepsilon \leqslant C \varepsilon .
$$

Остается проверить оценку

$$
h_{\mathscr{M}(w)}(t)=1+\frac{\sigma}{\theta} \int_{0}^{t} \operatorname{Re} f(\mathscr{N}(\mathscr{M}(w))) d \tau \geqslant \frac{\eta}{3 \mu} \theta^{\sigma} t^{\mu}
$$

для всех $t>0$. В силу условия (75) имеем

$$
\begin{aligned}
\frac{\sigma}{\theta} \int_{0}^{t} \operatorname{Re} & f(\mathscr{N}(\mathscr{M}(w))) d \tau=\frac{\sigma}{\theta} \int_{0}^{t} \operatorname{Re} f\left(\mathscr{N}\left(\theta G_{0}(\tau)\right)\right) d \tau \\
& +\frac{\sigma}{\theta} \int_{0}^{t} \operatorname{Re} f\left(\mathscr{N}(\mathscr{M}(w))-\mathscr{N}\left(\theta G_{0}(\tau)\right)\right) d \tau \geqslant \frac{\eta}{2 \mu} \theta^{\sigma} t^{\mu}+R(t)
\end{aligned}
$$


где $R(t)=\frac{\sigma}{\theta} \int_{0}^{t} \operatorname{Re} f\left(\mathscr{N}(\mathscr{M}(w))-\mathscr{N}\left(\theta G_{0}(\tau)\right)\right) d \tau$. Из оценки (77) получаем

$$
\begin{aligned}
|R(t)| \leqslant \frac{C}{\theta} & \left\|\mathscr{M}(w)-\mathscr{G}(t) v_{0}\right\|_{\mathbf{X}}\left(\|\mathscr{M}(w)\|_{\mathbf{X}}^{\sigma}+\left\|\mathscr{G}(t) v_{0}\right\|_{\mathbf{X}}^{\sigma}\right) \int_{0}^{t}\{\tau\}^{-\alpha}\langle\tau\rangle^{\mu-1} d \tau \\
+\frac{C}{\theta} & \left\|\langle t\rangle^{\gamma}\left(\mathscr{G}(t) v_{0}-\theta G_{0}(t)\right)\right\|_{\mathbf{X}}\left(\left\|\mathscr{G}(t) v_{0}\right\|_{\mathbf{X}}^{\sigma}+\left\|\theta G_{0}\right\|_{\mathbf{X}}^{\sigma}\right) \\
& \times \int_{0}^{t}\{\tau\}^{-\alpha}\langle\tau\rangle^{\mu-\gamma-1} d \tau \leqslant C \varepsilon^{\sigma}\langle\tau\rangle^{\mu} .
\end{aligned}
$$

В итоге в силу (81) находим

$$
h_{\mathscr{M}(w)}(t) \geqslant 1+\frac{\eta}{2 \mu} \theta^{\sigma} t^{\mu}-C \varepsilon^{\sigma}\langle\tau\rangle^{\mu} \geqslant \frac{\eta}{3 \mu} \theta^{\sigma} t^{\mu}
$$

для всех $t>0$, поскольку $\mu \in(0,1)$ мало. Итак, мы видим, что $\mathscr{M}$ отображает $\mathbf{B}$ в себя.

Теперь оценим разность $\mathscr{M}(v)-\mathscr{M}(w)$ :

$$
\begin{aligned}
\|\mathscr{M}(v)-\mathscr{M}(w)\|_{\mathbf{X}}= & \left\|\int_{0}^{t} \mathscr{G}(t-\tau)\left(\mathscr{K}(v(\tau)) \frac{1}{h_{v}(\tau)}-\mathscr{K}(w(\tau)) \frac{1}{h_{w}(\tau)}\right) d \tau\right\|_{\mathbf{X}} \\
\leqslant & \frac{C \mu}{\theta^{\sigma}}\left\|\int_{0}^{t} \tau^{-\mu}|\mathscr{G}(t-\tau)(\mathscr{K}(v(\tau))-\mathscr{K}(w(\tau)))| d \tau\right\|_{\mathbf{X}} \\
& +\frac{C \mu^{2}}{\theta^{2 \sigma}}\left\|\int_{0}^{t} \tau^{-2 \mu}|\mathscr{G}(t-\tau) \mathscr{K}(w(\tau))|\left|h_{v}(\tau)-h_{w}(\tau)\right| d \tau\right\|_{\mathbf{X}}
\end{aligned}
$$

откуда, пользуясь оценкой

$$
\begin{aligned}
\left|h_{v}(t)-h_{w}(t)\right| & \leqslant \frac{C}{\theta}\left|\int_{0}^{t} f(\mathscr{N}(v(\tau))-\mathscr{N}(w(\tau))) d \tau\right| \\
& \leqslant \frac{C}{\theta}\|v-w\|_{\mathbf{X}} \int_{0}^{t}\{\tau\}^{-\alpha}\langle\tau\rangle^{\mu-1} d \tau \leqslant \frac{C \varepsilon^{\sigma} t^{\mu}}{\mu \theta}\|v-w\|_{\mathbf{X}}
\end{aligned}
$$

(справедливой ввиду (77)), а также оценкой (78), получаем

$$
\begin{aligned}
\|\mathscr{M}(v)-\mathscr{M}(w)\|_{\mathbf{X}} & \leqslant C \frac{\mu}{\theta^{\sigma}}\|v-w\|_{\mathbf{X}}\left(\varepsilon^{\sigma}+\theta^{-1}\left\|\int_{0}^{t} \tau^{-\mu}|\mathscr{G}(t-\tau) \mathscr{K}(w(\tau))| d \tau\right\|_{\mathbf{X}}\right) \\
& \leqslant C \mu\|v-w\|_{\mathbf{X}} \leqslant \frac{1}{2}\|v-w\|_{\mathbf{X}}
\end{aligned}
$$

для любых $v, w \in \mathbf{B}$. Таким образом, $\mathscr{M}$ является сжимающим отображением в $\mathbf{B}$.

Поэтому существует единственное решение $v \in \mathbf{B}$ задачи Коши (74), удовлетворяющее оценкам

$$
\|v\|_{\mathbf{X}} \leqslant C \varepsilon, \quad\left\|v-\mathscr{G}(t) v_{0}\right\|_{\mathbf{x}} \leqslant C \mu \varepsilon, \quad h_{v}(t) \geqslant \frac{\eta}{3 \mu} \theta^{\sigma} t^{\mu} .
$$

В силу равенства $u(t, x)=v(t, x) e^{i \psi(t)} h_{v}^{-1 / \sigma}(t)$ приходим к существованию решения интегрального уравнения (79), удовлетворяющего оценке $\left\|t^{\mu / \sigma} u\right\|_{\mathbf{X}} \leqslant C \varepsilon$. Теорема 16 доказана. 
Теперь найдем асимптотику при больших временах для решений задачи Коши (74) с субкритической нелинейностью $\mathscr{N}(u)$ неконвективного типа. Назовем оператор

$$
\mathscr{G}_{0}(t) \phi=t^{-\frac{n}{2}} \int_{\mathbb{R}^{n}} \widetilde{G}_{0}\left((x-y) t^{-\frac{1}{2}}\right) \phi(y) d y
$$

автомодельным асимптотическим оператором, отвечающим оператору Грина $\mathscr{G}(t)$ в пространствах $\mathbf{X}$ и $\mathbf{Z}$, если при некотором $\gamma>0$ выполнена оценка

$$
\left\|\mathscr{G}_{0}(t) \phi\right\|_{\mathbf{X}}+\left\|\langle t\rangle^{\gamma}\left(\mathscr{G}(t)-\mathscr{G}_{0}(t)\right) \phi\right\|_{\mathbf{X}} \leqslant C\|\phi\|_{\mathbf{z}}
$$

для любых $\phi \in \mathbf{Z}$. Также предположим, что асимптотическое ядро $G_{0}(t, x)$ имеет автомодельный вид $G_{0}(t, x)=t^{-n / 2} \widetilde{G}_{0}\left(x t^{-1 / 2}\right)$ и

$$
f\left(\phi\left(x t^{-\frac{1}{2}}\right)\right)=t^{\frac{n}{2}} f(\phi x)
$$

для всех $t>0, \phi \in \mathbf{Z}$.

Фиксируем нормированное пространство $\mathbf{Q}$ функций, заданных на $\mathbb{R}^{n}$, и нормированное пространство $\widetilde{\mathbf{X}} \subset \mathbf{X}$ функций, заданных на $[0, \infty) \times \mathbb{R}^{n}$, так что норма в $\mathbf{Q}$ индуцирована нормой в $\widetilde{\mathbf{X}}$, т. е. $\|\phi\|_{\mathbf{Q}}=\left\|t^{-\alpha} \phi\left(x t^{-1 / \delta}\right)\right\|_{\widetilde{\mathbf{X}}}$ и $\|\phi\|_{\mathbf{X}} \leqslant$ $C\|\phi\|_{\tilde{\mathbf{X}}}$.

В следующей теореме устанавливается существование автомодельных решений для уравнения (74).

ТЕОрема 17. Пусть выполнены условия (76), (77) и (82), и пусть $\mu \in(0,1)$ в оценках (75) и (77) достаточно мало. Также предположим, что имеет место оченка

$$
\begin{aligned}
& \left\|\int_{0}^{t}\left|\mathscr{G}_{0}(t-\tau)(\mathscr{K}(v(\tau))-\mathscr{K}(w(\tau)))\right| \tau^{-\mu} d \tau\right\|_{\widetilde{\mathbf{X}}} \\
& \quad \leqslant C\|v-w\|_{\tilde{\mathbf{X}}}\left(\|v\|_{\tilde{\mathbf{X}}}+\|w\|_{\tilde{\mathbf{X}}}\right)^{\sigma}\left(1+\frac{\|v\|_{\tilde{\mathbf{X}}}+\|w\|_{\tilde{\mathbf{X}}}}{\theta}\right)
\end{aligned}
$$

для любых $v, w \in \widetilde{\mathbf{X}}$, удовлетворяющ, ус условию $f(v)=f(w)=1$, где $\sigma>0$ и $\mathscr{K}(v)=\mathscr{N}(v)-(v / f(v)) f(\mathscr{N}(v))$. Тогда существует единственное решение $V \in \mathbf{Q}$ интегрального уравнения

$$
V=\widetilde{G}_{0}-\frac{\mu}{\sigma \operatorname{Re} f(\mathscr{N}(V))} \int_{0}^{1} z^{-\mu} \mathscr{G}_{0}(1-z) \mathscr{K}\left(z^{-\alpha} V\left(x z^{-\frac{1}{\delta}}\right)\right) d z .
$$

ДокАзАтЕльСтво. Определим преобразование

$$
\mathscr{R}(V)=\widetilde{G}_{0}-\frac{\mu}{\sigma f(\mathscr{N}(V))} \int_{0}^{1} z^{-\mu} \mathscr{G}_{0}(1-z) \mathscr{K}\left(z^{-\alpha} V\left(x z^{-\frac{1}{\delta}}\right)\right) d z,
$$

для любой функции $V \in \mathbf{A}$, где

$$
\mathbf{A}=\left\{V \in \mathbf{Q}: f(V)=1,\|V\|_{\mathbf{Q}} \leqslant C,\left\|V-\widetilde{G}_{0}\right\|_{\mathbf{Q}} \leqslant C \mu, \operatorname{Re} f(\mathscr{N}(V)) \geqslant \frac{\eta}{3 \sigma}\right\} .
$$

Поскольку $f(V)=1$ по определению нелинейности $\mathscr{K}(V)$, то $f(\mathscr{K}(V))=0$. Откуда следует, что $f(\mathscr{R}(V))=f\left(\widetilde{G}_{0}\right)+f(\mathscr{K}(V))=1$. 
Заметим, что из условий (76) и (82) вытекает

$$
f(\mathscr{N}(V))=f\left(t^{-\alpha} \mathscr{N}\left(V\left(x t^{-\frac{1}{\delta}}\right)\right)\right)=t^{\alpha \sigma} f\left(\mathscr{N}\left(t^{-\alpha} V\left(x t^{-\frac{1}{\delta}}\right)\right)\right)
$$

а из $(75)$ с $\mu=1-\alpha \sigma$ следует, что $\operatorname{Re} f\left(\mathscr{N}\left(\widetilde{G}_{0}\right)\right) \geqslant \eta /(2 \sigma)>0$. Используя свойство автомодельности, обозначая $\xi=x t^{-1 / \delta}$ и делая замену переменных интегрирования $\tau=z t, y=y^{\prime} t^{1 / \delta}$, получаем

$$
\begin{aligned}
& \int_{0}^{t} \tau^{-\mu} \mathscr{G}_{0}(t-\tau, x) \mathscr{K}\left(\tau^{-\alpha} V\left(x \tau^{-\frac{1}{\delta}}\right)\right) d \tau \\
& =\int_{0}^{t} \tau^{-\mu}(t-\tau)^{-\frac{n}{\delta}} \int_{\mathbb{R}^{n}} \widetilde{G}_{0}\left((x-y)(t-\tau)^{-\frac{1}{\delta}}\right) \mathscr{K}\left(\tau^{-\alpha} V\left(y \tau^{-\frac{1}{\delta}}\right)\right) d y d \tau \\
& =t^{1-\mu-\sigma \alpha-\alpha} \int_{0}^{1} \frac{d z}{z^{\mu}}(1-z)^{-\frac{n}{\delta}} \int_{\mathbb{R}^{n}} \widetilde{G}_{0}\left(\left(\xi-y^{\prime}\right)(1-z)^{-\frac{1}{\delta}}\right) \mathscr{K}\left(z^{-\alpha} V\left(y^{\prime} z^{-\frac{1}{\delta}}\right)\right) d y^{\prime} \\
& =t^{-\alpha} \int_{0}^{1} z^{-\mu} \mathscr{G}_{0}\left(1-z, x t^{-\frac{1}{\delta}}\right) \mathscr{K}\left(z^{-\alpha} V\left(x z^{-\frac{1}{\delta}}\right)\right) d z
\end{aligned}
$$

поскольку $\mu=1-\alpha \sigma$. Учитывая оценку (83) и следующее соотношение между нормами: $\left\|t^{-\alpha} \phi\left(x t^{-1 / \delta}\right)\right\|_{\widetilde{\mathbf{X}}}=\|\phi(\xi)\|_{\mathbf{Q}}$, найдем

$$
\begin{aligned}
\left\|\mathscr{R}(V)-\widetilde{G}_{0}\right\|_{\mathbf{Q}} & =\frac{\mu}{\sigma|f(\mathscr{N}(V))|}\left\|\int_{0}^{1} z^{-\mu} \mathscr{G}_{0}(1-z) \mathscr{K}\left(z^{-\alpha} V\left(x z^{-\frac{1}{\delta}}\right)\right) d z\right\|_{\mathbf{Q}} \\
& =C \mu\left\|\int_{0}^{t} \tau^{-\mu} \mathscr{G}_{0}(t-\tau) \mathscr{K}\left(\tau^{-\alpha} V\left(x \tau^{-\frac{1}{\delta}}\right)\right) d \tau\right\|_{\widetilde{\mathbf{X}}} \\
& \leqslant C \mu\left\|t^{-\alpha} V\left(x t^{-\frac{1}{\delta}}\right)\right\|_{\widetilde{\mathbf{X}}}^{\sigma+1}=C \mu\|V\|_{\mathbf{Q}}^{\sigma+1} \leqslant C \mu
\end{aligned}
$$

и, в частности, имеем $\|\mathscr{R}(V)\|_{\mathbf{Q}} \leqslant\left\|\widetilde{G}_{0}\right\|_{\mathbf{Q}}+\left\|\mathscr{R}(V)-\widetilde{G}_{0}\right\|_{\mathbf{Q}} \leqslant C+C \mu \leqslant C$. Применяя условия (75), (77) и (82), получаем

$$
\begin{aligned}
\operatorname{Re} f(\mathscr{N}(V))= & t^{1-\mu} \operatorname{Re} f\left(\mathscr{N}\left(t^{-\alpha} \widetilde{G}_{0}\left(x t^{-\frac{1}{\delta}}\right)\right)\right) \\
& \quad+t^{1-\mu} \operatorname{Re} f\left(\mathscr{N}\left(t^{-\alpha} V\left(x t^{-\frac{1}{\delta}}\right)\right)-\mathscr{N}\left(t^{-\alpha} \widetilde{G}_{0}\left(x t^{-\frac{1}{\delta}}\right)\right)\right) \\
\geqslant & \frac{\eta}{2 \sigma}-\left\|V-\widetilde{G}_{0}\right\|_{\mathbf{Q}}\left(\|V\|_{\mathbf{Q}}^{\sigma}+\left\|\widetilde{G}_{0}\right\|_{\mathbf{Q}}^{\sigma}\right) \geqslant \frac{\eta}{2 \sigma}-C \mu \geqslant \frac{\eta}{3 \sigma} .
\end{aligned}
$$

Таким образом, $\mathscr{R}(V) \in \mathbf{A}$.

Теперь, обозначив $v(t, x)=t^{-\alpha} V\left(x t^{-1 / \delta}\right)$ и $w(t, x)=t^{-\alpha} W\left(x t^{-1 / \delta}\right)$, оценим разность $\mathscr{R}(V)-\mathscr{R}(W)$ :

$$
\begin{aligned}
\|\mathscr{R}(V)-\mathscr{R}(W)\|_{\mathbf{Q}} \\
=\frac{\mu}{\sigma}\left\|\int_{0}^{1} \mathscr{G}_{0}(1-z)\left(\frac{\mathscr{K}\left(z^{-\alpha} V\left(x z^{-\frac{1}{\delta}}\right)\right)}{\operatorname{Re} f(\mathscr{N}(V))}-\frac{\mathscr{K}\left(z^{-\alpha} W\left(x z^{-\frac{1}{\delta}}\right)\right)}{\operatorname{Re} f(\mathscr{N}(W))}\right) \frac{d z}{z^{\mu}}\right\|_{\mathbf{Q}} \\
=\frac{\mu}{\sigma}\left\|\int_{0}^{t} \tau^{-\mu}\left|\mathscr{G}_{0}(t-\tau)(\mathscr{K}(v(\tau))-\mathscr{K}(w(\tau)))\right| d \tau\right\|_{\tilde{\mathbf{X}}} \\
\quad+\frac{\mu}{\sigma}\left\|\int_{0}^{t} \tau^{-\mu}\left|\mathscr{G}_{0}(t-\tau) \mathscr{K}(w(\tau))\right||f(\mathscr{N}(v))-f(\mathscr{N}(w))| d \tau\right\|_{\tilde{\mathbf{X}}},
\end{aligned}
$$


откуда ввиду (77), (82) и (86), используя оценку $|f(\mathscr{N}(V))-f(\mathscr{N}(W))| \leqslant$ $C\|v-w\|_{\mathbf{Q}}$, получаем

$$
\begin{aligned}
\|\mathscr{R}(V)-\mathscr{R}(W)\|_{\mathbf{Q}} & \leqslant C \mu\|v-w\|_{\widetilde{\mathbf{X}}}\left(1+\left\|\int_{0}^{t} \tau^{-\mu}\left|\mathscr{G}_{0}(t-\tau) \mathscr{K}(w(\tau))\right| d \tau\right\|_{\widetilde{\mathbf{X}}}\right) \\
& \leqslant C \mu\|V-W\|_{\mathbf{Q}} \leqslant \frac{1}{2}\|V-W\|_{\mathbf{Q}} .
\end{aligned}
$$

Итак, отображение $\mathscr{R}(V)$ является сжимающим в А. Следовательно, существует единственное решение $V \in \mathbf{A}$ интегрального уравнения (84). Теорема 17 доказана.

В следующей теореме мы найдем асимптотику при больших временах решений задачи Коши (74) с субкритической неконвективной нелинейностью.

Теорема 18. Пусть линейный оператор $\mathscr{L}$ удовлетворяет условию $f(\mathscr{L} u)=0$ для любой $u \in \mathbf{X}$. Пусть нелинейность $\mathscr{N}(u)$ в уравнении $(74)$ является субкритической неконвективной и удовлетворяет (75) с достаточно малым $\mu \in(0,1)$. Предположим, что выполнены условия (76) и (77). Также предположим, что имеют место следующие оценки:

$$
\begin{aligned}
& \left\|\langle t\rangle^{\gamma} \int_{0}^{t}|\mathscr{G}(t-\tau)(\mathscr{K}(v(\tau))-\mathscr{K}(w(\tau)))| \tau^{-\mu} d \tau\right\|_{\mathbf{X}} \\
& \quad \leqslant C\left\|\langle t\rangle^{\gamma}(v(t)-w(t))\right\|_{\mathbf{X}}\left(\|v\|_{\mathbf{X}}+\|w\|_{\mathbf{X}}\right)^{\sigma}\left(1+\frac{\|v\|_{\mathbf{X}}+\|w\|_{\mathbf{X}}}{\theta}\right), \\
& \left\|\langle t\rangle^{\gamma} \int_{0}^{t}|\mathscr{G}(t-\tau) \mathscr{K}(v(\tau))| \tau^{-\mu-\gamma} d \tau\right\|_{\mathbf{X}} \leqslant C\|v\|_{\mathbf{X}}^{\sigma+1}, \\
& \left\|\langle t\rangle^{\gamma} \int_{0}^{t}\left(\mathscr{G}(t-\tau)-\mathscr{G}_{0}(t-\tau)\right) \mathscr{K}(v(\tau)) \tau^{-\mu} d \tau\right\|_{\mathbf{X}} \leqslant C\|v\|_{\mathbf{X}}^{\sigma+1}
\end{aligned}
$$

для любых $v, w \in \mathbf{X}$, удовлетворяющих условию $f(v)=f(w)=\theta>0$, где $\sigma>0, \mathscr{K}(v)=\mathscr{N}(v)-(v / \theta) f(\mathscr{N}(v))$, a $\mathscr{G}_{0}-$ асимптотический автомодельный оператор для оператора Грина $\mathscr{G}$ в пространствах $\mathbf{X}$ и $\mathbf{Z}$. Пусть начальные возмущения $u_{0} \in \mathbf{Z}$ достаточно маль: $\left\|u_{0}\right\|_{\mathbf{Z}} \leqslant \varepsilon, u$ имеют среднее значение $\theta \equiv\left|f\left(u_{0}\right)\right| \geqslant C \varepsilon>0$ для некоторого $C>0$. Тогда существуют числа $A, \omega$ и функция $V \in \mathbf{Q}$ (которая является единственным решением интегрального уравнения (84)) такие, что для решения $и \in \mathbf{X}$ задачи Коши (74) справедлива следующая асимптотическая оченка:

$$
\left\|\langle t\rangle^{\gamma+\frac{\mu}{\sigma}}\left(u-A t^{-1 / \sigma} e^{i \omega \log t} V\left(x t^{-\frac{1}{\delta}}\right)\right)\right\|_{\mathbf{X}} \leqslant C
$$

с некоторым $\gamma>0$.

ДокАзАТЕльство. Сначала установим оценку

$$
\left\|\langle t\rangle^{\gamma}(v(t)-w(t))\right\|_{\mathbf{X}} \leqslant C
$$

с некоторым $\gamma \in(0, \mu)$, где функция $v$ удовлетворяет интегральному уравнению (79), а функция $w$ имеет вид $w(t)=t^{-\alpha} \theta V\left(x t^{-1 / \delta}\right)$, где $V$ является 
единственным решением интегрального уравнения (84), так что

$$
w(t)=\theta G_{0}(t)-\int_{0}^{t} \mathscr{G}_{0}(t-\tau) \mathscr{K}(w(\tau)) \frac{d \tau}{\tilde{h}_{w}(\tau)},
$$

где $G_{0}(t)=t^{-\alpha} \widetilde{G}_{0}\left(x t^{-1 / \delta}\right)$ и $\tilde{h}_{w}(t)=\sigma(\mu \theta)^{-1} t \operatorname{Re} f(\mathscr{N}(w(t)))$. Заметим, что ввиду связи норм справедливо неравенство $\|w\|_{\mathbf{X}} \leqslant C\|w\|_{\widetilde{\mathbf{X}}}=C \theta\|V\|_{\mathbf{Q}} \leqslant C \theta$. Откуда в силу (78) и (90) получаем

$$
\begin{aligned}
& \left\|\langle t\rangle^{\gamma}(v(t)-w(t))\right\|_{\mathbf{X}} \leqslant\left\|\langle t\rangle^{\gamma}\left(\mathscr{G}(t) v_{0}-\theta G_{0}(t)\right)\right\|_{\mathbf{X}} \\
& \quad+\left\|\langle t\rangle^{\gamma} \int_{0}^{t}\left(\mathscr{G}_{0}(t-\tau) \mathscr{K}(w(\tau)) \frac{1}{\tilde{h}_{w}(\tau)}-\mathscr{G}(t-\tau) \mathscr{K}(v(\tau)) \frac{1}{h_{v}(\tau)}\right) d \tau\right\|_{\mathbf{X}} .
\end{aligned}
$$

Воспользовавшись определением асимптотического ядра, оценим первое слагаемое в правой части (91): $\left\|\langle t\rangle^{\gamma}\left(\mathscr{G}(t) v_{0}-\theta G_{0}(t)\right)\right\|_{\mathbf{X}} \leqslant C\left\|v_{0}\right\|_{\mathbf{Z}} \leqslant C \varepsilon$. Затем перепишем (91) в виде

$$
\begin{aligned}
& \left\|\langle t\rangle^{\gamma}(v(t)-w(t))\right\|_{\mathbf{X}} \leqslant C \varepsilon+\left\|\langle t\rangle^{\gamma} \int_{0}^{t}\left(\mathscr{G}(t-\tau)-\mathscr{G}_{0}(t-\tau)\right) \mathscr{K}(w(\tau)) \frac{d \tau}{\tilde{h}_{w}(\tau)}\right\|_{\mathbf{X}} \\
& \quad+\left\|\langle t\rangle^{\gamma} \int_{0}^{t} \mathscr{G}(t-\tau)(\mathscr{K}(v(\tau))-\mathscr{K}(w(\tau))) \frac{d \tau}{\tilde{h}_{w}(\tau)}\right\|_{\mathbf{X}} \\
& \quad+\left\|\langle t\rangle^{\gamma} \int_{0}^{t}\left(\frac{1}{h_{v}(\tau)}-\frac{1}{\tilde{h}_{w}(\tau)}\right) \mathscr{G}(t-\tau) \mathscr{K}(v(\tau)) d \tau\right\|_{\mathbf{X}} \equiv C \varepsilon+I_{1}+I_{2}+I_{3} .
\end{aligned}
$$

Ввиду (87) получим

$$
I_{1} \leqslant \frac{C \mu}{\theta^{\sigma}}\left\|\langle t\rangle^{\gamma} \int_{0}^{t}\left(\mathscr{G}(t-\tau)-\mathscr{G}_{0}(t-\tau)\right) \mathscr{K}(v(\tau)) \tau^{-\mu} d \tau\right\|_{\mathbf{X}} \leqslant \frac{C \mu}{\theta^{\sigma}}\|v\|_{\mathbf{X}}^{\sigma+1} \leqslant C \mu \varepsilon .
$$

Величину $I_{2}$ в (92) оценим с помощью (85):

$$
\begin{aligned}
I_{2} & \leqslant \frac{C \mu}{\theta^{\sigma}}\left\|\langle t\rangle^{\gamma} \int_{0}^{t}|\mathscr{G}(t-\tau)(\mathscr{K}(v(\tau))-\mathscr{K}(w(\tau)))| \tau^{-\mu} d \tau\right\|_{\mathbf{X}} \\
& \leqslant \frac{C \mu}{\theta^{\sigma}}\left\|\langle t\rangle^{\gamma}(v(t)-w(t))\right\|_{\mathbf{X}}\left(\|v\|_{\mathbf{X}}+\|w\|_{\mathbf{X}}\right)^{\sigma}\left(1+\frac{\|v\|_{\mathbf{X}}+\|w\|_{\mathbf{X}}}{\theta}\right) \\
& \leqslant C \mu\left\|\langle t\rangle^{\gamma}(v(t)-w(t))\right\|_{\mathbf{X}} .
\end{aligned}
$$

Теперь оценим разность $h_{v}(t)-\tilde{h}_{w}(t)$. Поскольку

$$
\begin{aligned}
\tilde{h}_{w}(t) & =\frac{\sigma}{\mu \theta} t \operatorname{Re} f(\mathscr{N}(w(t)))=\frac{\sigma}{\mu \theta} t^{\mu} \operatorname{Re} f(\mathscr{N}(V)) \\
& =\frac{\sigma}{\theta} \int_{0}^{t} t^{\mu-1} \operatorname{Re} f(\mathscr{N}(V)) d \tau=\frac{\sigma}{\theta} \int_{0}^{t} \operatorname{Re} f(\mathscr{N}(w(\tau))) d \tau
\end{aligned}
$$


то в силу условия (77) найдем

$$
\begin{aligned}
\left|h_{v}(t)-\tilde{h}_{w}(t)\right| & \leqslant 1+\frac{\sigma}{\theta} \int_{0}^{t}|f(\mathscr{N}(v(\tau))-\mathscr{N}(w(\tau)))| d \tau \\
& \leqslant 1+\frac{C}{\theta} \varepsilon^{\sigma}\left\|\langle t\rangle^{\gamma}(v(t)-w(t))\right\|_{\mathbf{X}} \int_{0}^{t}\{\tau\}^{-\alpha}\langle\tau\rangle^{\mu-\gamma-1} d \tau \\
& \leqslant 1+\frac{C}{\theta(\mu-\gamma)} \varepsilon^{\sigma} t^{\mu-\gamma}\left\|\langle t\rangle^{\gamma}(v(t)-w(t))\right\|_{\mathbf{X}},
\end{aligned}
$$

так как $\gamma<\mu$. Таким образом, благодаря (86) получим

$$
\begin{aligned}
I_{3}= & \left\|\langle t\rangle^{\gamma} \int_{0}^{t}\left(\frac{1}{h_{v}(\tau)}-\frac{1}{\tilde{h}_{w}(\tau)}\right) \mathscr{G}(t-\tau) \mathscr{K}(v(\tau)) d \tau\right\|_{\mathbf{X}} \\
\leqslant & C \mu^{2} \theta^{-2 \sigma}\left\|\langle t\rangle^{\gamma} \int_{0}^{t}\left|h_{v}(\tau)-\tilde{h}_{w}(\tau)\right||\mathscr{G}(t-\tau) \mathscr{K}(v(\tau))| \tau^{-2 \mu} d \tau\right\|_{\mathbf{X}} \\
\leqslant & C \mu^{2} \theta^{-2 \sigma}\left\|\langle t\rangle^{\gamma} \int_{0}^{t}|\mathscr{G}(t-\tau) \mathscr{K}(v(\tau))| \tau^{-2 \mu} d \tau\right\|_{\mathbf{X}} \\
& \quad+\frac{C \mu}{\theta^{1+\sigma}}\left\|\langle t\rangle^{\gamma}(v(t)-w(t))\right\|_{\mathbf{X}}\left\|\langle t\rangle^{\gamma} \int_{0}^{t}|\mathscr{G}(t-\tau) \mathscr{K}(v(\tau))| \tau^{-\mu-\gamma} d \tau\right\|_{\mathbf{X}} \\
\leqslant & C \mu^{2} \theta^{1-\sigma}+C \mu\left\|\langle t\rangle^{\gamma}(v(t)-w(t))\right\|_{\mathbf{X}} \cdot
\end{aligned}
$$

Подставляя оценки для $I_{1}, I_{2}, I_{3}$ в $(92)$, находим

$$
\left\|\langle t\rangle^{\gamma}(v(t)-w(t))\right\|_{\mathbf{X}} \leqslant C \varepsilon+C \mu^{2} \theta^{1-\sigma}+C \mu\left\|\langle t\rangle^{\gamma}(v(t)-w(t))\right\|_{\mathbf{X}} .
$$

Следовательно, используя тот факт, что $\mu$ достаточно мало, приходим к оценке (89). В силу (80) имеем

$$
\psi(t)=\arg f\left(u_{0}\right)-\frac{1}{\theta} \int_{0}^{t} \frac{1}{h_{v}(\tau)} \operatorname{Im} f(\mathscr{N}(v(\tau))) d \tau .
$$

Благодаря оценке (93) получаем

$$
\left|h_{v}(t)-\beta \theta^{\sigma}\langle t\rangle^{\mu}\right| \leqslant C\langle t\rangle^{\mu-\gamma} \quad \text { для любых } t>0,
$$

где $\beta=(\sigma / \mu) \operatorname{Re} f(\mathscr{N}(V))>\eta /(2 \mu)>0$. Кроме того, ввиду (77) справедливо неравенство $\left|\operatorname{Im} f(\mathscr{N}(v(\tau)))-\chi \theta^{\sigma+1}\langle t\rangle^{\mu-1}\right| \leqslant C \varepsilon^{\sigma+1}\langle t\rangle^{\mu-\gamma}$ для всех $t>0$, где $\chi=\operatorname{Im} f(\mathscr{N}(V))$. Подстановка последних оценок в (94) дает

$$
\begin{aligned}
\psi(t)=- & \frac{\chi}{\beta} \int_{0}^{t}\langle\tau\rangle^{-1} d \tau+\arg f\left(u_{0}\right)-\frac{1}{\theta} \int_{0}^{\infty}\left(\frac{1}{h_{v}(\tau)} \operatorname{Im} f(\mathscr{N}(v(\tau)))-\frac{\chi}{\beta}\langle\tau\rangle^{-1}\right) d \tau \\
& +\frac{1}{\theta} \int_{t}^{\infty}\left(\frac{1}{h_{v}(\tau)} \operatorname{Im} f(\mathscr{N}(v(\tau)))-\frac{\chi}{\beta}\langle\tau\rangle^{-1}\right) d \tau \\
= & \omega \log t+\Psi+O\left(\int_{t}^{\infty}\langle\tau\rangle^{-1-\gamma} d \tau\right)=\omega \log t+\Psi+O\left(t^{-\gamma}\right)
\end{aligned}
$$

где $\omega=-\chi / \beta$ и

$$
\Psi \equiv \arg f\left(u_{0}\right)-\frac{1}{\theta} \int_{0}^{\infty}\left(\frac{1}{h_{v}(\tau)} \operatorname{Im} f(\mathscr{N}(v(\tau)))-\frac{\chi}{\beta}\langle\tau\rangle^{-1}\right) d \tau
$$


Поэтому, учитывая формулу $u(t, x)=e^{i \psi(t)} h_{v}^{-1 / \sigma}(t) v(t, x)$ и асимптотические оценки (89), (95) и (96), приходим к асимптотике (88), в которой $A=\beta^{-1 / \sigma} e^{i \Psi}$. Теорема 18 доказана.

Применение теорем 16-18 к доказательству существования глобального во времени решения задачи Коши для уравнения типа Соболева со степенной нелинейностью (23) в субкритическом случае $0<\sigma<2 / n$ дает следующий результат (см. [64]). Обозначим $\widetilde{G}_{0}(x)=(4 \pi \alpha)^{-n / 2} e^{-|x|^{2} /(4 \alpha)}$.

Теорема 19. Пусть $0<\sigma<2 / n$. Предположим, что началъные возмущения $u_{0} \in \mathbf{L}^{\infty} \cap \mathbf{L}^{1, a}, a \in(0,1)$, достаточно маль: $\left\|u_{0}\right\|_{\mathbf{L}^{\infty}}+\left\|u_{0}\right\|_{\mathbf{L}^{1, a}} \leqslant \varepsilon$, u $\lambda \theta \leqslant-C \varepsilon<0$, где $\theta=\int_{\mathbb{R}^{n}} u_{0}(x) d x$. Предположим также, что величина $\sigma$ достаточно близка $к$ значению $2 / n$, так что $2 / n-\sigma \leqslant C \varepsilon^{\sigma}$. Тогда задача Коши (23) имеет единственное глобальное во времени решение $u(t) \in$ $\mathbf{C}\left([0, \infty) ; \mathbf{L}^{\infty} \cap \mathbf{L}^{1, a}\right)$. Это решение удовлетворяет оценке $\|u(t)\|_{\mathbf{L}^{\infty}} \leqslant C\langle t\rangle^{-1 / \sigma}$ при всех $t>0$. Более того, существуют число $A$ и функиия $V \in \mathbf{L}^{1, a} \cap \mathbf{L}^{\infty}$ такие, что справедлива асимптотическая формула $u(t, x)=A t^{-1 / \sigma} V\left(x t^{-1 / 2}\right)+$ $O\left(t^{-1 / \sigma-\gamma}\right)$ при $t \rightarrow \infty$ равномерно по $x \in \mathbb{R}^{n}$, где $\gamma=(1 / 2) \min (a, 1-n \sigma / 2)$, a $V(\xi)$ является решением интегрального уравнения

$$
V(\xi)=\widetilde{G}_{0}(\xi)-\frac{1}{\beta} \int_{0}^{1} \frac{d z}{z(1-z)^{\frac{n}{2}}} \int_{\mathbb{R}^{n}} \widetilde{G}_{0}\left(\frac{\xi-y z^{\frac{1}{2}}}{\sqrt{1-z}}\right) F(y) d y,
$$

əдe

$$
\begin{gathered}
\beta=\sigma\left(1-\frac{n}{2} \sigma\right)^{-1} \int_{\mathbb{R}^{n}} V^{1+\sigma}(y) d y, \\
F(y)=V^{1+\sigma}(y)-V(y) \int_{\mathbb{R}^{n}} V^{1+\sigma}(\xi) d \xi .
\end{gathered}
$$

6.2. Большие начальные данные. В этом пункте мы освободимся от условия малости начальных данных и установим глобальное во времени существование решений задачи Коши (23) с субкритической степенью нелинейности $\sigma \in(0,2 / n)$. По-прежнему будем предполагать, что $\sigma \in(0,2 / n)$ достаточно близко к $2 / n$. Следующий результат доказан в работе [64].

Tеорема 20. Пусть $\lambda<0, n=1,2$. Предположим, что $u_{0} \in \mathbf{W}_{\infty}^{2}\left(\mathbb{R}^{n}\right) \cap$ $\mathbf{W}_{1}^{2, a}\left(\mathbb{R}^{n}\right), 0<a \leqslant 1, u \theta=\int_{\mathbb{R}^{n}} u_{0}(x) d x \neq 0$. Предположим также, что $2 / n-\varepsilon<\sigma<2 / n$, где $\varepsilon>0$ достаточно мало, так что заведомо имеют место неравенства $\sigma>1$ для $n=1$ и $\sigma>3 / 4$ для $n=2$. Тогда существует единственное глобальное во времени решение $u(t, x) \in \mathbf{C}\left([0, \infty) ; \mathbf{C}\left(\mathbb{R}^{n}\right) \cap\right.$ $\left.\mathbf{L}^{\infty}\left(\mathbb{R}^{n}\right) \cap \mathbf{L}^{1, a}\left(\mathbb{R}^{n}\right)\right)$ задачи Коши (23). Это решение имеет асимптотику

$$
u(t, x)=A t^{-1 / \sigma} V\left(x t^{-1 / 2}\right)+O\left(t^{-1 / \sigma-\gamma}\right)
$$

при $t \rightarrow \infty$ равномерно по $x \in \mathbb{R}^{n}$, где $A, V$ те же, что и в теореме 19. 


\section{7. Субкритическая конвективная нелинейность}

В этом разделе мы изучим асимптотическое поведение при больших временах решений $u(t, x)$ задачи Коши для уравнения типа Соболева с конвективной нелинейностью:

$$
\begin{gathered}
\partial_{t}\left(u-u_{x x}\right)-u_{x x}+|u|^{\sigma} u_{x}=0, \quad x \in \mathbb{R}, \quad t>1, \\
u(1, x)=u_{0}(x), \quad x \in \mathbb{R},
\end{gathered}
$$

в субкритическом случае $\sigma \in(0,1)$. Мы представим асимптотику решений задачи (97) как произведение волны разряжения и ударной волны.

Сначала рассмотрим вспомогательную задачу построения волны разряжения для уравнения Хопфа:

$$
\begin{aligned}
\varphi_{t}+\varphi^{\sigma} \varphi_{x} & =0, \quad x \in \mathbb{R}, \quad t>0, \\
\varphi(0, x) & =\varphi_{0}(x), \quad x \in \mathbb{R},
\end{aligned}
$$

где начальное возмущение $\varphi_{0}(x) \in \mathbf{C}^{2}(\mathbb{R})$ монотонно возрастает $\left(0<\varphi_{0}^{\prime}(x)<C\right.$ для всех $x \in \mathbb{R}), \varphi_{0}(x) \rightarrow 0$ при $x \rightarrow-\infty, \varphi_{0}(x) \rightarrow 1$ при $x \rightarrow+\infty$. Решение задачи (98) можно записать в виде $\varphi(t, \chi(t, \xi))=\varphi_{0}(\xi)$, где характеристики $\chi(t, \xi)$ равны $\xi+t \varphi_{0}^{\sigma}(\xi)$ при $\xi \in \mathbb{R}, t>0$. Заметим, что

$$
\begin{aligned}
\varphi_{x}(t, \chi(t, \xi)) & =\frac{\varphi_{0}^{\prime}(\xi)}{1+t \sigma \varphi_{0}^{\sigma-1}(\xi) \varphi_{0}^{\prime}(\xi)}>0 \\
\varphi_{x x}(t, \chi(t, \xi)) & =\frac{\varphi_{0}^{\prime \prime}(\xi)}{\left(1+t \sigma \varphi_{0}^{\sigma-1}(\xi) \varphi_{0}^{\prime}(\xi)\right)^{2}}-\frac{\varphi_{0}^{\prime}(\xi) t \sigma\left(\varphi_{0}^{\sigma-1}(\xi) \varphi_{0}^{\prime}(\xi)\right)^{\prime}}{\left(1+t \sigma \varphi_{0}^{\sigma-1}(\xi) \varphi_{0}^{\prime}(\xi)\right)^{3}}
\end{aligned}
$$

для всех $\xi \in \mathbb{R}, t>0$. Предположим также, что начальное возмущение $\varphi_{0}(x)$ таково, что при $t \rightarrow \infty$ справедливы асимптотические оценки

$$
\left\|\varphi_{x}(t)\right\|_{\mathbf{L}^{\infty}} \leqslant C\langle t\rangle^{-1}, \quad\left\|\varphi_{x x}(t)+\varphi_{x x t}(t)\right\|_{\mathbf{L}^{2}} \leqslant C\langle t\rangle^{-1-\gamma}
$$

с некоторым $\gamma>0$. Условия (99) выполнены, например, если взять начальное возмущение $\varphi_{0}(x) \in \mathbf{C}^{2}(\mathbb{R})$, имеющее следующее асимптотическое поведение: $\varphi_{0}(\xi)=\vartheta(\xi)+O\left(|\xi|^{-\beta}\right), \frac{d^{k}}{d \xi^{k}} \varphi_{0}(\xi)=O\left(|\xi|^{-\beta-k}\right), \quad k=1,2$, при $\xi \rightarrow \pm \infty$, где $\beta>0$ и $\vartheta(\xi)=1$ для $\xi \geqslant 0$ и $\vartheta(\xi)=0$ для $\xi<0$.

Приведем сначала достаточно общий результат о сходимости при $t \rightarrow \infty$ решений $u(t, x)$ задачи $(97)$ к волне разряжения $\varphi(t, x)$.

Теорема 21. Пусть $u_{0}-\varphi_{0} \in \mathbf{L}^{2}(\mathbb{R})$. Предположим, что начальное возмущение $\varphi_{0}(x) \in \mathbf{C}^{2}(\mathbb{R})$ удовлетворяет условиям $(99)$. Тогда $u(t, x)=\varphi(t, x)+$ $o(1)$ при $t \rightarrow \infty$ равномерно по $x \in \mathbb{R}$.

ДоказАТЕЛЬСтво. Для разности $w=u-\varphi$ получим задачу Коши

$$
\begin{gathered}
\partial_{t}\left(w-w_{x x}\right)-w_{x x}+\frac{1}{\sigma+1} \partial_{x}\left(|w+\varphi|^{\sigma+1}\right)-\varphi^{\sigma} \varphi_{x}-\left(\varphi_{x x}+\varphi_{x x t}\right)=0, \\
w(0, x)=w_{0}(x)
\end{gathered}
$$


где $w_{0}(x)=u_{0}(x)-\varphi_{0}(x) \in \mathbf{L}^{2}(\mathbb{R})$. Нетрудно доказать существование единственного решения

$$
w(t, x) \in \mathbf{C}\left([0, \infty) ; \mathbf{L}^{2}(\mathbb{R})\right) \cap \mathbf{C}\left((0, \infty) ; \mathbf{H}^{2}(\mathbb{R})\right) \cap \mathbf{C}^{1}\left((0, \infty) ; \mathbf{L}^{\infty}(\mathbb{R}) \cap \mathbf{L}^{2}(\mathbb{R})\right)
$$

задачи (100). Умножив уравнение (100) на $w$ и проинтегрировав по всему пространству $\mathbb{R}$, получим априорную оценку энергетического типа

$$
\begin{gathered}
\frac{1}{2} \frac{d}{d t}\|w\|_{\mathbf{H}^{1}}^{2}+\int_{\mathbb{R}}\left(\frac{w}{\sigma+1} \partial_{x}\left(|w+\varphi|^{\sigma+1}\right)-w \varphi^{\sigma} \varphi_{x}\right) d x \\
=-\left\|w_{x}\right\|_{\mathbf{L}^{2}}^{2}+\int_{\mathbb{R}} w\left(\varphi_{x x}+\varphi_{x x t}\right) d x .
\end{gathered}
$$

Заметим, что (см. [84])

$$
\begin{aligned}
& \int_{\mathbb{R}}(\left.\frac{w}{\sigma+1} \partial_{x}\left(|w+\varphi|^{\sigma+1}\right)-w \varphi^{\sigma} \varphi_{x}\right) d x \\
& \quad= \frac{1}{1+\sigma} \int_{\mathbb{R}}\left(|w+\varphi|^{\sigma+1}-\varphi^{\sigma+1}-(1+\sigma) \varphi^{\sigma} w\right) \varphi_{x} d x \\
&=\sigma \int_{\mathbb{R}} d x w^{2} \varphi_{x} \int_{0}^{1}|\varphi+\lambda w|^{\sigma-1}(1-\lambda) d \lambda \geqslant 0 .
\end{aligned}
$$

Откуда в силу неравенства Коши и оценок (99) имеем $\frac{d}{d t}\|w\|_{\mathbf{H}^{1}}^{2}+2\left\|w_{x}\right\|_{\mathbf{L}^{2}}^{2} \leqslant$ $C\langle t\rangle^{-1-\gamma}\|w\|_{\mathbf{L}^{2}}$. Интегрируя это неравенство по времени, получим

$$
\|w(t)\|_{\mathbf{H}^{1}} \leqslant C \quad \text { и } \quad \int_{0}^{\infty}\left\|w_{x}(\tau)\right\|_{\mathbf{L}^{2}}^{2} d \tau \leqslant C,
$$

значит, существует последовательность $t_{k} \rightarrow \infty$ такая, что $\left\|w_{x}\left(t_{k}\right)\right\|_{\mathbf{L}^{2}} \rightarrow 0$. Для того чтобы получить априорную оценку энергетического типа, умножим уравнение $(100)$ на $w_{x x}$ и проинтегрируем по всему пространству $\mathbb{R}$ :

$$
\begin{gathered}
\frac{1}{2} \frac{d}{d t}\left\|w_{x}\right\|_{\mathbf{H}^{1}}^{2}+\int_{\mathbb{R}} \frac{|w+\varphi|^{\sigma+1}}{w+\varphi} w_{x} w_{x x} d x+\int_{\mathbb{R}}\left(\frac{|w+\varphi|^{\sigma+1}}{w+\varphi}-\varphi^{\sigma}\right) w_{x x} \varphi_{x} d x \\
=-\left\|w_{x x}\right\|_{\mathbf{L}^{2}}^{2}+\int_{\mathbb{R}} w_{x x}\left(\varphi_{x x}+\varphi_{x x t}\right) d x .
\end{gathered}
$$

Используя для интегралов в левой части оценки

$$
\begin{aligned}
\int_{\mathbb{R}} \frac{|w+\varphi|^{\sigma+1}}{w+\varphi} w_{x} w_{x x} d x & \leqslant C\left(\|w\|_{\mathbf{L}^{\infty}}^{\sigma}+1\right)\left\|w_{x}\right\|_{\mathbf{L}^{2}}\left\|w_{x x}\right\|_{\mathbf{L}^{2}}, \\
\int_{\mathbb{R}}\left(\frac{|w+\varphi|^{\sigma+1}}{w+\varphi}-\varphi^{\sigma}\right) w_{x x} \varphi_{x} d x & \leqslant C\|w\|_{\mathbf{L}^{\infty}}^{\sigma}\left\|w_{x x}\right\|_{\mathbf{L}^{2}}\left\|\varphi_{x}\right\|_{\mathbf{L}^{2}} \\
& \leqslant C\left\|w_{x}\right\|_{\mathbf{L}^{2}}^{\frac{\sigma}{2}}\left\|w_{x x}\right\|_{\mathbf{L}^{2}}\left\|\varphi_{x}\right\|_{\mathbf{L}^{2}}
\end{aligned}
$$

и учитывая, что $\left\|\varphi_{x}\right\|_{\mathbf{L}^{2}} \leqslant\left\|\varphi_{x}\right\|_{\mathbf{L}^{\infty}}^{1 / 2}\left\|\varphi_{x}\right\|_{\mathbf{L}^{1}}^{1 / 2}=\left\|\varphi_{x}\right\|_{\mathbf{L}^{\infty}}^{1 / 2} \leqslant C\langle t\rangle^{-1 / 2}$ (так как $\left.\int \varphi_{x} d x=1\right)$, получаем

$$
\begin{aligned}
\frac{d}{d t}\left\|w_{x}\right\|_{\mathbf{H}^{1}}^{2} & \leqslant-\left\|w_{x x}\right\|_{\mathbf{L}^{2}}^{2}+C\left(\langle t\rangle^{-1-\gamma}+\left\|w_{x}\right\|_{\mathbf{L}^{2}}+\langle t\rangle^{-\frac{1}{2}}\|w\|_{\mathbf{L}^{\infty}}^{\sigma}\right)\left\|w_{x x}\right\|_{\mathbf{L}^{2}} \\
& \leqslant C\langle t\rangle^{-1-\gamma}+C\left\|w_{x}\right\|_{\mathbf{L}^{2}}^{2} .
\end{aligned}
$$


Интегрируя по времени, находим

$$
\left\|w_{x}(t)\right\|_{\mathbf{H}^{1}}^{2} \leqslant\left\|w_{x}(T)\right\|_{\mathbf{H}^{1}}^{2}+C \int_{T}^{\infty}\langle\tau\rangle^{-1-\gamma} d \tau+C \int_{T}^{\infty}\left\|w_{x}(\tau)\right\|_{\mathbf{L}^{2}}^{2} d \tau .
$$

Откуда следует, что $\left\|w_{x}(t)\right\|_{\mathbf{L}^{2}} \rightarrow 0$ при $t \rightarrow \infty$, поэтому $\|w(t)\|_{\mathbf{L}^{\infty}} \leqslant C\|w(t)\|_{\mathbf{L}^{2}}^{1 / 2} \times$ $\left\|w_{x}(t)\right\|_{\mathbf{L}^{2}}^{1 / 2} \rightarrow 0$ при $t \rightarrow \infty$. Теорема 21 доказана.

Теперь мы наложим более сильные ограничения на начальные возмущения $u_{0}(x)$ и вычислим более точно асимптотику при больших временах решения $u(t, x)$ задачи Коши (97) с граничными условиями типа волны разряжения. Предположим, что начальные возмущения $u_{0}(x)$ монотонно возрастают от нуля до единицы и медленно меняются так, что старшие производные малы по сравнению с младшими. Более точно, предположим, что начальные данные $u_{0}(x) \in \mathbf{C}^{3}(\mathbb{R})$ удовлетворяют оценкам

$$
\begin{aligned}
\frac{d}{d x} u_{0}^{\sigma}(x) & \leqslant \varepsilon^{\frac{1}{1-\gamma}}\left(u_{0}^{\sigma}(x)\right)^{\frac{2}{1-\gamma}}, \\
\frac{d^{2}}{d x^{2}} u_{0}^{\sigma}(x) & \leqslant \varepsilon\left(\frac{d}{d x} u_{0}^{\sigma}(x)\right)^{\frac{3+\gamma}{2}}, \\
\frac{d^{3}}{d x^{3}} u_{0}^{\sigma}(x) & \leqslant \varepsilon\left(\frac{d}{d x} u_{0}^{\sigma}(x)\right)^{2+\gamma}
\end{aligned}
$$

для всех $x \in \mathbb{R}$, где $\gamma \in(0,1 / 2)$ и $\varepsilon>0$ достаточно мало. Например, мы можем взять начальные возмущения вида $u_{0}(x)=C\left(\int_{-\infty}^{x} \varepsilon^{2}\left(1+\varepsilon^{4} \xi^{2}\right)^{\gamma-1} d \xi\right)^{1 / \sigma}$, где $C>0$ таково, что $u_{0}(+\infty)=1$. Отметим, что такие начальные данные $u_{0}(x)$ убывают со скоростью $O\left(|x|^{(2 \gamma-1) / \sigma}\right)$ при $x \rightarrow-\infty$.

Из теоремы 21 нам известно, что решения задачи (97) приближаются с течением времени к решениям уравнения Хопфа (98). Значит, нелинейность в уравнении (97) доминирует над линейными членами, и асимптотика решений уравнения (97) в основном определяется членами $u_{t}$ и $(\sigma+1)^{-1} \partial_{x}\left(|u|^{\sigma+1}\right)$. Исходя из этого, будем решать уравнение (97) методом характеристик. Определим характеристики $\chi(t, \xi)$ как решения задачи Коши

$$
\begin{gathered}
\partial_{t} \chi=|u|^{\sigma-1} u(t, \chi)-\frac{u_{\chi \chi}}{u_{\chi}}-\frac{u_{\chi \chi_{t}}}{u_{\chi}}, \quad \xi \in \mathbb{R}, \quad t>0, \\
\chi(0, \xi)=\xi, \quad \xi \in \mathbb{R} .
\end{gathered}
$$

Тогда в силу уравнения (97) получаем для новой функции $w(t, \xi)=u(t, \chi(t, \xi))$ уравнение

$$
\begin{aligned}
w_{t}(t, \xi) & =u_{t}+u_{\chi} \chi_{t}=u_{t}+u_{\chi}\left(|u|^{\sigma-1} u-\frac{u_{\chi \chi}}{u_{\chi}}-\frac{u_{\chi \chi_{t}}}{u_{\chi}}\right) \\
& =\partial_{t}\left(u-u_{\chi \chi}\right)-u_{\chi \chi}+\frac{1}{\sigma+1} \partial_{\chi}\left(|u|^{\sigma+1}\right)=0 .
\end{aligned}
$$

Значит, $w(t, \xi)=u_{0}(\xi)$ для всех $t>0$ и $\xi \in \mathbb{R}$. Прямое вычисление дает

$$
\partial_{\chi} u=\frac{u_{0}^{\prime}(\xi)}{\chi_{\xi}(t, \xi)}, \quad \partial_{\chi}^{2} u=\frac{u_{0}^{\prime \prime}(\xi)}{\chi_{\xi}^{2}(t, \xi)}-\frac{u_{0}^{\prime}(\xi) \chi_{\xi \xi}(t, \xi)}{\chi_{\xi}^{3}(t, \xi)}
$$


поэтому уравнение для характеристики $\chi(t, \xi)$ принимает вид

$$
\partial_{t} \chi=u_{0}^{\sigma}(\xi)-\frac{1}{u_{0}^{\prime}(\xi)} \partial_{\xi}\left(\frac{u_{0}^{\prime}(\xi)}{\chi_{\xi}(t, \xi)}\right)-\frac{\chi_{\xi}(t, \xi)}{u_{0}^{\prime}(\xi)} \partial_{t}\left(\frac{1}{\chi_{\xi}(t, \xi)} \partial_{\xi}\left(\frac{u_{0}^{\prime}(\xi)}{\chi_{\xi}(t, \xi)}\right)\right) .
$$

Сделаем замену независимой переменной $\eta=u_{0}^{\sigma}(\xi)$, тогда (в силу предположения $\left.u_{0}^{\prime}(\xi)>0\right)$ числовая ось $\xi \in \mathbb{R}$ взаимно однозначно отображается на отрезок $(0,1)$. Обозначим $m(\eta)=\frac{\partial \eta}{\partial \xi}=\sigma u_{0}^{\sigma-1}(\xi) u_{0}^{\prime}(\xi)$ и $Z(t, \eta)=\frac{m(\eta)}{\chi_{\xi}(t, \xi)}$. Тогда имеем

$$
\begin{aligned}
\partial_{t} \chi & =\eta-\eta^{1-\frac{1}{\sigma}} \partial_{\eta}\left(\eta^{\frac{1}{\sigma}-1} Z\right)-\frac{1}{Z} \partial_{t}\left(Z \eta^{1-\frac{1}{\sigma}} \partial_{\eta}\left(\eta^{\frac{1}{\sigma}-1} Z\right)\right) \\
& =\eta-\partial_{\eta} Z-\kappa \frac{Z}{\eta}-\frac{1}{Z} \partial_{t}\left(Z \partial_{\eta} Z+\frac{2 \kappa}{\eta} Z\right)
\end{aligned}
$$

где $\kappa=1 / \sigma-1$, откуда следует, что

$$
\begin{aligned}
\partial_{t} \chi_{\xi}(t, \xi) & =m(\eta)-m(\eta) \partial_{\eta}\left(\partial_{\eta} Z+\kappa \frac{Z}{\eta}+\frac{1}{Z} \partial_{t}\left(Z \partial_{\eta} Z+\frac{2 \kappa}{\eta} Z\right)\right) \\
& =m(\eta)\left(1-\partial_{\eta}^{2} Z-\kappa \partial_{\eta}\left(\frac{Z}{\eta}\right)-\partial_{\eta}\left(\frac{1}{Z} \partial_{t}\left(Z \partial_{\eta} Z+\frac{2 \kappa}{\eta} Z\right)\right)\right) .
\end{aligned}
$$

Таким образом, для $Z(t, \eta)$ получаем уравнение

$$
\begin{aligned}
\partial_{t} Z=- & Z^{2} \frac{1}{m(\eta)} \partial_{t} \chi_{\xi}(t, \xi)=-Z^{2}+Z^{2} Z_{\eta \eta}+\frac{\kappa}{\eta} Z^{2} Z_{\eta}-\frac{\kappa}{\eta^{2}} Z^{3} \\
& \quad-Z_{t}\left(\frac{3}{2} Z_{\eta}^{2}+Z Z_{\eta \eta}+\frac{4 \kappa}{\eta} Z_{\eta}+\frac{2 \kappa}{\eta^{2}} Z\right)+\partial_{t}\left(\frac{1}{2} Z Z_{\eta}^{2}+Z^{2} Z_{\eta \eta}+\frac{2 \kappa}{\eta} Z Z_{\eta}\right) \\
= & -Z^{2}(1-A),
\end{aligned}
$$

где

$$
\begin{aligned}
A=Z_{\eta \eta} & +\frac{\kappa}{\eta} Z_{\eta}-\frac{\kappa}{\eta^{2}} Z+\frac{1}{Z} Z_{\eta} Z_{\eta t}-\frac{1}{Z^{2}} Z_{t} Z_{\eta}^{2} \\
& +\frac{1}{Z} Z_{t} Z_{\eta \eta}+Z_{\eta \eta t}+\frac{2 \kappa}{Z \eta} Z_{\eta t}-\frac{2 \kappa}{Z \eta^{2}} Z_{t}-\frac{2 \kappa}{\eta Z^{2}} Z_{t} Z_{\eta} .
\end{aligned}
$$

Итак, функция $Z(t, \eta)$ является решением следующей начально-краевой задачи:

$$
\begin{gathered}
Z_{t}=-Z^{2}(1-A), \quad \eta \in(0,1), \quad t>0, \\
Z(0, \eta)=m(\eta), \quad \eta \in(0,1), \\
\left.Z^{k-\frac{3}{2}} \partial_{\eta}^{k} Z\right|_{\eta=0,1}=0, \quad t>0, \quad k=1,2,
\end{gathered}
$$

поскольку ввиду (101) мы предполагаем, что $u_{0}(\xi)$ такова, что $\partial_{\eta}^{k} m(\eta)=o\left(\eta^{2-k}\right)$ при $\eta \rightarrow 0$ для $k=1,2$.

Ввиду существования единственного решения $u(t, x)$ задачи (97) существует единственное глобальное во времени решение $Z(\tau, \eta) \in \mathbf{C}\left([0, \infty) ; \mathbf{C}^{2}(0,1)\right) \cap$ $\left.\mathbf{C}^{1}((0, \infty) ; \mathbf{C}(0,1))\right)$ начально-краевой задачи (102). Интегрирование уравнения (102) по времени $t>0$ дает следующее представление:

$$
Z(t, \eta)=m(\eta)\left(1+m(\eta)\left(t+\int_{0}^{t} A(\tau, \eta) d \tau\right)\right)^{-1}
$$


Следуя работе [67], получаем такой результат:

ТЕОРема 22. Пусть для начального возмущения $u_{0}(x)$ выполнены условия (101) с достаточно мальм $\varepsilon>0$. Тогда имеет место оценка

$$
\sup _{\eta \in(0,1)}\left|\int_{1}^{t} A(\tau, \eta) d \tau\right| \leqslant C \varepsilon t^{1-\gamma}
$$

для всех $t \geqslant 1$, где $\gamma \in(0,1 / 2)$ взято из условия (101).

Сначала рассмотрим вспомогательную задачу построения волны разряжения для уравнения (97)

$$
\begin{gathered}
\partial_{t}\left(\varphi-\varphi_{x x}\right)-\varphi_{x x}+|\varphi|^{\sigma} \varphi_{x}=0, \quad x \in \mathbb{R}, \quad t>1, \\
\varphi(1, x)=\varphi_{0}(x), \quad x \in \mathbb{R},
\end{gathered}
$$

где начальное возмущение $\varphi_{0}(x) \in \mathbf{C}^{2}(\mathbb{R})$ монотонно возрастает $\left(0<\varphi_{0}^{\prime}(x)<C\right.$ для всех $x \in \mathbb{R}), \varphi_{0}(x) \rightarrow 0$ при $x \rightarrow-\infty$ и $\varphi_{0}(x) \rightarrow 1$ при $x \rightarrow+\infty$. Определим теперь решение $r(t, x)$ типа ударной волны:

$$
\begin{gathered}
r_{t}+\varphi^{\sigma}|r|^{\sigma} r_{x}-r_{x x}-\frac{1}{\varphi} \varphi_{x x} r_{t}+\frac{2}{\varphi}\left(\left(\varphi^{\sigma} \varphi_{x}\right)_{x}-\varphi_{x}\right) r_{x}-\frac{2}{\varphi} \varphi_{x} r_{t x} \\
+\varphi^{\sigma-1} \varphi_{x} r_{x x}-r_{t x x}+\varphi^{\sigma-1} \varphi_{x}\left(|r|^{\sigma}-1\right) r=0, \quad x \in \mathbb{R}, \quad t>1, \\
r(1, x)=r_{0}(x), \quad x \in \mathbb{R},
\end{gathered}
$$

где начальное возмущение $r_{0}(x)=u_{0}(x) / \varphi_{0}(x)$ удовлетворяет граничным условиям типа ударной волны $r_{0}(x) \rightarrow 1$ при $x \rightarrow-\infty$ и $r_{0}(x) \rightarrow 0$ при $x \rightarrow+\infty$, т. е. $\varphi_{0}(x) \approx u_{0}(x)$ при $x \rightarrow-\infty$ и $r_{0}(x) \approx u_{0}(x)$ при $x \rightarrow+\infty$. Тогда в силу $(104)$ и (105) функция $u=\varphi r$ удовлетворяет исходной задаче Коши (97).

Сделав замену переменных

$$
r(t, x)=w(t, y), \quad x=y t^{\frac{1}{2}-\alpha}+\frac{2 a_{0}}{1+2 \alpha} t^{\frac{1}{2}+\alpha}+\int_{1}^{t} a_{1}(\tau) \tau^{-\frac{1}{2}-\alpha} d \tau
$$

$\left(a_{0}, a_{1}(t)\right.$ и $\alpha$ выберем позже), получим

$$
\begin{gathered}
w_{t}+\sum_{k=1}^{5} J_{k}=0, \quad y \in \mathbb{R}, \quad t>1, \\
w(1, y)=r_{0}(y), \quad y \in \mathbb{R},
\end{gathered}
$$

где

$$
\begin{aligned}
& J_{1}=t^{2 \alpha-1} \partial_{y}\left(\frac{B}{1+\sigma}|w|^{\sigma} w-a_{0} w-w_{y}\right), \\
& J_{2}=-t^{-1}\left(\nu y+a_{1}(t)\right) w_{y}, \\
& J_{3}=-\frac{\varphi_{x x}}{\varphi} w_{t}-2 t^{\alpha-\frac{1}{2}} \frac{\varphi_{x}}{\varphi} w_{t y}-t^{2 \alpha-1} w_{t y y}, \\
& J_{4}=q_{1} w_{y}+q_{2} w_{y y}+q_{3} w_{y y y}, \\
& J_{5}=\varphi^{\sigma} \frac{\varphi_{x}}{\varphi}\left(|w|^{\sigma}-1\right) w+t^{2 \alpha-1}\left(\varphi^{\sigma} t^{\frac{1}{2}-\alpha}-B\right)|w|^{\sigma} w_{y},
\end{aligned}
$$


где $\nu=1 / 2-\alpha, a(t)=a_{0}+a_{1}(t) t^{-2 \alpha}$,

$$
\begin{aligned}
& q_{1}=\left(\nu y+a t^{2 \alpha}\right) t^{-1} \frac{\varphi_{x x}}{\varphi}+\frac{2}{\varphi} t^{\alpha-\frac{1}{2}}\left(\varphi^{\sigma} \varphi_{x}\right)_{x}+2\left(\nu t^{\alpha-\frac{3}{2}}-t^{\alpha-\frac{1}{2}}\right) \frac{\varphi_{x}}{\varphi} \\
& q_{2}=2\left(\nu y+a t^{2 \alpha}\right) t^{\alpha-\frac{3}{2}} \frac{\varphi_{x}}{\varphi}+t^{2 \alpha-1} \varphi^{\sigma} \frac{\varphi_{x}}{\varphi}+(1-2 \alpha) t^{2 \alpha-2}, \\
& q_{3}=t^{2 \alpha-2}\left(\nu y+t^{2 \alpha} a(t)\right) .
\end{aligned}
$$

Чтобы сократить расходящееся слагаемое $J_{1}$ в левой части уравнения (106), будем искать первое приближение $\Phi(y)$ как решение уравнения $\partial_{y}\left(\frac{B}{1+\sigma}|\Phi|^{\sigma} \Phi-\right.$ $\left.a_{0} \Phi-\Phi^{\prime}\right)=0$ с граничными условиями $\Phi(y) \rightarrow 1$ при $y \rightarrow-\infty$ и $\Phi(y) \rightarrow 0$ при $y \rightarrow+\infty$. Решая это уравнение с заданными граничными условиями, находим $a_{0}=B(1+\sigma)^{-1}$ и единственное с точностью до сдвига решение $\Phi(y)=\left(1+e^{h y}\right)^{-1 / \sigma}$, где $h=a_{0} \sigma$. Рассмотрим кривую $y=0$, т. е. $x_{f}(t)=$ $\int_{1}^{t} a(\tau) \tau^{\alpha-1 / 2} d \tau$, будем называть ее фронтом волны. Имеем

$$
x_{f}(t)=\frac{2 a_{0}}{1+2 \alpha} t^{\alpha+\frac{1}{2}}+O\left(t^{\frac{1}{2}-\alpha}\right)
$$

при $t \rightarrow \infty$. Определим $\xi_{0}(t)$ из условия $\chi\left(t, \xi_{0}(t)\right)=x_{f}(t)$, тогда $\varphi\left(t, x_{f}(t)\right)=$ $\varphi_{0}\left(\xi_{0}(t)\right)$, и в силу $(107)$, для функции $\xi_{0}(t)$ получим из уравнения $\chi(t, \xi)=$ $\xi+t \varphi_{0}^{\sigma}(\xi)+O\left(t^{1 / 2-\alpha}\right)$ соотношение

$$
\xi_{0}+\varphi_{0}^{\sigma}\left(\xi_{0}\right) t=\frac{2 a_{0}}{1+2 \alpha} t^{\alpha+\frac{1}{2}}+O\left(t^{\frac{1}{2}-\alpha}\right)
$$

при $t \rightarrow \infty$, поэтому

$$
t^{\frac{1}{2}-\alpha} \varphi^{\sigma}\left(t, x_{f}(t)\right)-\frac{2 a_{0}}{1+2 \alpha}=O\left(t^{-2 \alpha}\right)+O\left(t^{-\frac{1}{2}-\alpha} \xi_{0}\right)
$$

при $t \rightarrow \infty$. Обозначим $B=\lim _{t \rightarrow \infty} t^{1 / 2-\alpha} \varphi^{\sigma}\left(t, x_{f}(t)\right)$, тогда имеем $B=$ $2 a_{0} /(1+2 \alpha)$, откуда ввиду полученного ранее соотношения $a_{0}=B /(1+\sigma)$ находим $\alpha=1 / 2-\sigma /(1+\sigma)$.

Предположим, что начальное возмущение $\varphi_{0}(\xi)$ имеет асимптотику

$$
\frac{d^{k}}{d \xi^{k}} \varphi_{0}(\xi)=b_{1}|\xi|^{-\frac{1}{\sigma}-k}+O\left(|\xi|^{-\frac{1}{\sigma}-1-k}\right) \quad \text { при } \xi \rightarrow-\infty, k=0,1,2,3,
$$

тогда ввиду (108) имеем уравнение

$$
b_{1}^{\sigma}\left|\xi_{0}(t)\right|^{-1}=B t^{\alpha-\frac{1}{2}}+O\left(t^{-\frac{1}{2}-\alpha}\right)-\xi_{0} t^{-1}+O\left(\left|\xi_{0}\right|^{-1-\sigma}\right),
$$

откуда сначала находим оценку $\xi_{0}(t)=O\left(t^{1 / 2-\alpha}\right)$, а затем асимптотику $\xi_{0}(t)=$ $-t^{1 / 2-\alpha} b_{1}^{\sigma} / B+O\left(t^{1 / 2-3 \alpha}\right)$. Таким образом, $\varphi^{\sigma}\left(t, x_{f}(t)\right)=B t^{\alpha-1 / 2}+O\left(t^{-1 / 2-\alpha}\right)$, $\varphi\left(t, x_{f}(t)\right)=B^{1 / \sigma} t^{-1 /(1+\sigma)}+O\left(t^{-1 /(1+\sigma)-2 \alpha}\right)$.

При $t \rightarrow \infty$ справедливы соотношения

$$
\varphi_{x}\left(t, x_{f}(t)\right)=\frac{\varphi_{0}^{\prime}\left(\xi_{0}\right)}{1+t \sigma \varphi_{0}^{\sigma-1}\left(\xi_{0}\right) \varphi_{0}^{\prime}\left(\xi_{0}\right)}=O\left(\frac{b_{2}\left|\xi_{0}\right|^{-\frac{1}{\sigma}-1}}{1+t \sigma b_{1}^{\sigma-1} b_{2} \xi_{0}^{-2}}\right)=O\left(t^{-1-2 \alpha}\right)
$$


и $\varphi_{x}(t, x)=O\left(t^{-1}\right)$. Поскольку $x-x_{f}(t)=y t^{1 / 2-\alpha}$, по формуле Тейлора имеем $\varphi(t, x)=\varphi\left(t, x_{f}(t)\right)+\left(x-x_{f}(t)\right) \varphi_{x}(t, \tilde{x})=B^{\frac{1}{\sigma}} t^{-\frac{1}{1+\sigma}}+O\left(t^{-\frac{1}{1+\sigma}-2 \alpha}\right)+O\left(y t^{-\frac{1}{2}-\alpha}\right)$.

Таким образом, получаем

$$
t^{\frac{1}{2}-\alpha} \varphi^{\sigma}(t, x)-B=O\left(y t^{-2 \alpha}\right) \quad \text { и } \quad \frac{\varphi_{x}\left(t, x_{f}(t)\right)}{\varphi\left(t, x_{f}(t)\right)}=O\left(t^{-\frac{1}{2}-\alpha}\right) .
$$

Заметим, что $\varphi_{x}(t, x) / \varphi(t, x)=O\left(t^{-1 / 2}\right)$ и $\varphi_{x x}(t, x) / \varphi(t, x)=O\left(t^{-1}\right)$, откуда по формуле Тейлора

$$
\frac{\varphi_{x}(t, x)}{\varphi(t, x)}=\frac{\varphi_{x}\left(t, x_{f}(t)\right)}{\varphi\left(t, x_{f}(t)\right)}+\left(x-x_{f}(t)\right)\left(\frac{\varphi_{x x}(t, \tilde{x})}{\varphi(t, \tilde{x})}-\frac{\varphi_{x}^{2}(t, \tilde{x})}{\varphi^{2}(t, \tilde{x})}\right)=O\left((1+|y|) t^{-\frac{1}{2}-a}\right)
$$

при $t \rightarrow \infty$. Отметим также оценки $q_{2}=O\left((1+|y|) t^{-1}\right), q_{3}=O\left((1+|y|) t^{2 \alpha-2}\right)$ и $q_{4}=\nu y t^{2 \alpha-2}+O\left(t^{4 \alpha-2}\right)$.

Докажем теперь, что решение $w(t, y)$ уравнения (106) сходится к приближенному решению $\Phi(y)$ при $t \rightarrow \infty$. Ввиду (106) получаем для разности $v=w-\Phi_{0}$ уравнение

$$
\begin{gathered}
\partial_{t}\left(v-t^{2 \alpha-1} v_{y y}-\frac{\varphi_{x x}}{\varphi} v-2 t^{\alpha-\frac{1}{2}} \frac{\varphi_{x}}{\varphi} v_{y}\right)+t^{2 \alpha-1} \frac{B}{1+\sigma} \partial_{y}\left(|v+\Phi|^{\sigma}(v+\Phi)-\Phi^{\sigma+1}\right) \\
-t^{-1}\left(\nu y+a t^{2 \alpha}\right) v_{y}-t^{2 \alpha-1}\left(1+2 \nu t^{-1}\right) v_{y y}+R_{1}+R_{2}+R_{3}=0,
\end{gathered}
$$

где

$$
\begin{aligned}
& R_{1}=\left(q_{2}-t^{-1}\left(\nu y+a_{1}(t)\right)\right) \Phi^{\prime}+q_{3} \Phi^{\prime \prime}+q_{4} \Phi^{\prime \prime \prime}, \\
& R_{2}=\varphi^{\sigma} \frac{\varphi_{x}}{\varphi}\left(|v+\Phi|^{\sigma}-1\right)(v+\Phi)+t^{2 \alpha-1}\left(\varphi^{\sigma} t^{\frac{1}{2}-\alpha}-B\right)|v+\Phi|^{\sigma}\left(v_{y}+\Phi^{\prime}\right), \\
& R_{3}=\left(\frac{\varphi_{x x}}{\varphi}\right)_{t} v+2\left(t^{\alpha-\frac{1}{2}} \frac{\varphi_{x}}{\varphi}\right)_{t} v_{y}+q_{2} v_{y}+q_{3} v_{y y}+q_{4} v_{y y y} .
\end{aligned}
$$

Потребуем, чтобы $a_{1}(t)$ удовлетворяло условию

$$
\int_{-\infty}^{\infty}\left(R_{1}+R_{2}+R_{3}\right) d y^{\prime}=0
$$

Тогда

$$
F(t, y)=\int_{-\infty}^{y}\left(R_{1}+R_{2}+R_{3}\right) d y^{\prime}=-\int_{y}^{\infty}\left(R_{1}+R_{2}+R_{3}\right) d y^{\prime}
$$

Имеем оценку $\left|\int_{-\infty}^{y} R_{1} d y^{\prime}\right| \leqslant C h^{-1} t^{-1-2 \alpha}\left(1+y^{2}\right) e^{-2 L(y)}$, где $L(y)=a_{0}|y| / 2$ при $y>0$ и $L(y)=h|y| / 2$ при $y<0 \quad\left(h=\sigma a_{0}\right)$. Интегрирование уравнения (109) по у дает

$$
\begin{gathered}
\partial_{t}\left(V-t^{2 \alpha-1} V_{y y}-\int_{-\infty}^{y}\left(\frac{\varphi_{x x}}{\varphi} V_{y}+2 t^{\alpha-\frac{1}{2}} \frac{\varphi_{x}}{\varphi} V_{y y}\right) d y\right) \\
-t^{-1} \nu y V_{y}+\nu t^{-1} V+t^{2 \alpha-1} a_{0}\left(\left|V_{y}+\Phi\right|^{\sigma}\left(V_{y}+\Phi\right)-\Phi^{\sigma+1}\right) \\
-t^{2 \alpha-1} a V_{y}-t^{2 \alpha-1}\left(1+2 \nu t^{-1}\right) V_{y y}+F=0, \quad y \in \mathbb{R}, \quad t>1, \\
V(0, x)=V_{0}(x), \quad x \in \mathbb{R},
\end{gathered}
$$


где

$$
V(t, y)=\int_{-\infty}^{y} v\left(t, y^{\prime}\right) d y^{\prime} \quad \text { и } \quad F=\int_{-\infty}^{y}\left(R_{1}+R_{2}+R_{3}\right) d y^{\prime} .
$$

Заметим, что $V_{0}(x)=\int_{-\infty}^{x}\left(w_{0}\left(x^{\prime}\right)-W\left(1, x^{\prime}\right)\right) d x^{\prime} \rightarrow 0$ при $x \rightarrow \pm \infty$.

Предположим теперь, что начальные возмущения $w_{0}(x)$ для задачи $(110)$ достаточно близки к приближенной ударной волне $W(1, x)$, так что

$$
e^{L(x)} \int_{-\infty}^{x}\left(w_{0}(\xi)-W(1, \xi)\right) d \xi \in \mathbf{H}^{2},
$$

где $L(x)=a_{0}|x| / 2$ при $x>0$ и $L(x)=h|x| / 2$ при $x<0\left(h=\sigma a_{0}, a_{0}=B /(1+\sigma)\right)$. Обозначим $\alpha=1 / 2-\sigma /(1+\sigma), \sigma \in(0,1)$.

Докажем следующий результат.

Теорема 23. Предположим, что начальное возмущение $u_{0}(\xi)$ имеет следующую асимптотику:

$$
\frac{d^{k}}{d \xi^{k}} u_{0}(\xi)=b_{1}|\xi|^{-\frac{1}{\sigma}-k}+O\left(|\xi|^{-\frac{1}{\sigma}-1-k}\right) \quad \text { npu } \xi \rightarrow-\infty, \quad k=0,1,2,3 .
$$

Пусть функиия $\varphi_{0}(x)$ монотонна $\left(\varphi_{0}^{\prime}(x)>0\right.$ nри всех $\left.x \in \mathbb{R}\right) u \varphi_{0}(x)=u_{0}(x)+$ $o(1)$ при $x \rightarrow-\infty$. Пусть начальные возмущения $u_{0}(x)$ близки $к$ ударной волне $W(0, x), \max$ чmo

$$
\left\|e^{L(x)} \int_{-\infty}^{x}\left(\frac{u_{0}(\xi)}{\varphi_{0}(\xi)}-W(1, \xi)\right) d \xi\right\|_{\mathbf{H}^{2}} \leqslant \varepsilon,
$$

где $\varepsilon>0$ достаточно мало. Предположим, что величина В достаточно велика: $B \geqslant C / \varepsilon$. Тогда единственное решение $u(t, x)$ задачи Коши (97) имеет при $t \rightarrow \infty$ асимптотическое представление

$$
u(t, x)=\varphi(t, x) \Phi(y)+O\left(t^{-\frac{1}{2}-\alpha}\right),
$$

равномерное по $x \in \mathbb{R}$.

ДоказАтЕльство. Сделаем замену $g(t, y)=V(t, y) e^{\theta(y)}$. Функцию $\theta(y)$ определим из уравнения $\theta^{\prime}(y)=(1 / 2)\left(a_{0}-B \Phi^{\sigma}(y)\right)$, тогда

$$
\theta(y)=L(y)+\frac{B}{2 h} \log \left(1+e^{-h|y|}\right)
$$

где $L(x)=a_{0}|x| / 2$ при $x>0$ и $L(x)=h|x| / 2$ при $x<0$ и $h=\sigma a_{0}, a_{0}=B /(1+\sigma)$. Из уравнения (110) найдем

$$
\begin{gathered}
G_{t}+t^{2 \alpha-1} a_{0} e^{\theta}\left(\left|\left(g_{y}-\theta^{\prime} g\right) e^{-\theta}+\Phi\right|^{\sigma}\left(\left(g_{y}-\theta^{\prime} g\right) e^{-\theta}+\Phi\right)-\Phi^{\sigma+1}\right) \\
-t^{2 \alpha-1}\left(1+2 \nu t^{-1}\right) g_{y y}-\phi g_{y}+\psi g+e^{\theta} F=0,
\end{gathered}
$$


где

$$
\begin{gathered}
G=g-t^{2 \alpha-1}\left(g_{y y}-2 \theta^{\prime} g_{y}+\left(\theta^{\prime 2}-\theta^{\prime \prime}\right) g\right)-e^{\theta} \int_{-\infty}^{y}\left(\frac{\varphi_{x x}}{\varphi}\left(g_{y}-\theta^{\prime} g\right) e^{-\theta}\right. \\
\left.\quad+2 t^{\alpha-\frac{1}{2}} \frac{\varphi_{x}}{\varphi}\left(g_{y y}-2 \theta^{\prime} g_{y}+\left(\theta^{\prime 2}-\theta^{\prime \prime}\right) g\right) e^{-\theta}\right) d y \\
\phi(t, y)=t^{2 \alpha-1} a+t^{-1} \nu y-2 \theta^{\prime} t^{2 \alpha-1}\left(1+2 \nu t^{-1}\right), \\
\psi(t, y)=t^{2 \alpha-1} a \theta^{\prime}+t^{-1} \nu y \theta^{\prime}+\nu t^{-1}-t^{2 \alpha-1}\left(1+2 \nu t^{-1}\right)\left(\theta^{\prime 2}-\theta^{\prime \prime}\right) .
\end{gathered}
$$

Докажем, что $\|G(t)\|_{\mathbf{L}^{2}}<C t^{-2 \alpha}$ при всех $t>0$. Предположим противное, тогда в силу непрерывности найдется $T>0$ такое, что

$$
\|G(t)\|_{\mathbf{L}^{2}} \leqslant C t^{-2 \alpha}
$$

при всех $t \in[0, T]$. Применим энергетический метод. Умножим уравнение (111) на $G$ и после этого проинтегрируем результат по $y$, получим оценку

$$
\begin{gathered}
\frac{d}{d t}\|G(t)\|_{\mathbf{L}^{2}}^{2} \leqslant- \\
t^{2 \alpha-1} a_{0}\left(e^{\theta} G,\left|\Phi+\left(g_{y}-\theta^{\prime} g\right) e^{-\theta}\right|^{\sigma}\left(\Phi+\left(g_{y}-\theta^{\prime} g\right) e^{-\theta}\right)-\Phi^{\sigma+1}\right)_{\mathbf{L}^{2}} \\
+t^{2 \alpha-1}\left(1+2 \nu t^{-1}\right)\left(G, g_{y y}\right)_{\mathbf{L}^{2}}+\left(G, \phi g_{y}-\psi g\right)_{\mathbf{L}^{2}}+\left\|e^{\theta} G F\right\|_{\mathbf{L}^{1}} .
\end{gathered}
$$

Ввиду оценок

$$
\begin{gathered}
t^{2 \alpha-1} a_{0} e^{\theta}\left(\left|\Phi+\left(g_{y}-\theta^{\prime} g\right) e^{-\theta}\right|^{\sigma}\left(\Phi+\left(g_{y}-\theta^{\prime} g\right) e^{-\theta}\right)-\Phi^{\sigma+1}\right) \\
=t^{2 \alpha-1} B \Phi^{\sigma}\left(g_{y}-\theta^{\prime} g\right)+O\left(\left(g_{y}-\theta^{\prime} g\right) t^{2 \alpha-1-2 \sigma \alpha}\right)
\end{gathered}
$$

и

$$
\begin{aligned}
-t^{2 \alpha-1} B\left(\theta^{\prime} \Phi^{\sigma}+\frac{1}{2}\left(\Phi^{\sigma}\right)^{\prime}\right)+\psi+\frac{1}{2} \phi_{y} \\
=\frac{B^{2}(1-\sigma)}{4(1+\sigma)} t^{2 \alpha-1}\left(\frac{\sigma^{2}}{1-\sigma^{2}}+\left(1-\Phi^{\sigma}\right)^{2}\right) \geqslant C B^{2} t^{2 \alpha-1}
\end{aligned}
$$

при всех $y \in \mathbb{R}$ и соотношений $\Phi^{\sigma}=\left(1+e^{h y}\right)^{-1}$ и $\theta^{\prime}=(1 / 2)\left(a_{0}-B \Phi^{\sigma}\right)$, а также ввиду оценок $\varphi_{x} / \varphi=O\left((1+|y|) t^{-1 / 2-a}\right)$ и (112), дающих оценку $\left\|e^{\theta} F(t)\right\|_{\mathbf{L}^{2}} \leqslant$ $C t^{-1}$, получаем

$$
\frac{d}{d t}\|G(t)\|_{\mathbf{L}^{2}} \leqslant-C B^{2} t^{2 \alpha-1}\|G(t)\|_{\mathbf{L}^{2}}+C t^{-1},
$$

откуда, интегрируя по времени, находим, что $\|G(t)\|_{\mathbf{L}^{2}} \leqslant C \varepsilon t^{-2 \alpha}$ при всех $t \geqslant 0$. Аналогично оценим производную $G_{y}$. Имеем

$$
\begin{gathered}
G_{y t}-\phi g_{y y}+\partial_{y} t^{2 \alpha-1} a_{0} e^{\theta}\left(\left|\left(g_{y}-\theta^{\prime} g\right) e^{-\theta}+\Phi\right|^{\sigma}\left(\left(g_{y}-\theta^{\prime} g\right) e^{-\theta}+\Phi\right)-\Phi^{\sigma+1}\right) \\
-t^{2 \alpha-1}\left(1+2 \nu t^{-1}\right) g_{y y y}+\psi_{y} g+\left(\psi-\phi_{y}\right) g_{y}+\partial_{y}\left(e^{\theta} F\right)=0 .
\end{gathered}
$$

Применяя энергетический метод, умножим (114) на $G_{y}$ и проинтегрируем по $y$; в итоге получим

$$
\begin{gathered}
\frac{d}{d t}\left\|G_{y}(t)\right\|_{\mathbf{L}^{2}}^{2} \leqslant-\left(G_{y}, t^{2 \alpha-1} e^{\theta} \theta^{\prime} a_{0}\left(\left|\Phi+\left(g_{y}-\theta^{\prime} g\right) e^{-\theta}\right|^{\sigma}\left(\Phi+\left(g_{y}-\theta^{\prime} g\right) e^{-\theta}\right)\right.\right. \\
\left.\left.-\Phi^{\sigma+1}-(1+\sigma) \Phi^{\sigma}\left(g_{y}-\theta^{\prime} g\right) e^{-\theta}\right)\right)_{\mathbf{L}^{2}}
\end{gathered}
$$




$$
\begin{aligned}
& -\left(G_{y}, t^{2 \alpha-1} \Phi_{y} e^{\theta} B\left(\left|\Phi+\left(g_{y}-\theta^{\prime} g\right) e^{-\theta}\right|^{\sigma}-\Phi^{\sigma}-\sigma \Phi^{\sigma-1}\left(g_{y}-\theta^{\prime} g\right) e^{-\theta}\right)\right)_{\mathbf{L}^{2}} \\
& +\left(G_{y}, t^{2 \alpha-1} B\left(\left|\Phi+\left(g_{y}-\theta^{\prime} g\right) e^{-\theta}\right|^{\sigma}-\Phi^{\sigma}\right)\left(2 \theta^{\prime} g_{y}-\left(\theta^{2}-\theta^{\prime \prime}\right) g\right)\right)_{\mathbf{L}^{2}} \\
& -\left(G_{y},\left(\psi-\phi_{y}-t^{2 \alpha-1} B|\Phi|^{\sigma} \theta^{\prime}+t^{2 \alpha-1} \sigma B \Phi^{\sigma-1} \Phi_{y}\right) g_{y}\right)_{\mathbf{L}^{2}} \\
& -\left(G_{y},\left(\psi_{y}-t^{2 \alpha-1} B|\Phi|^{\sigma} \theta^{\prime 2}-t^{2 \alpha-1} \sigma B \Phi^{\sigma-1} \Phi_{y} \theta^{\prime}\right) g\right)_{\mathbf{L}^{2}}-\left(G_{y}, \partial_{y}\left(e^{\theta} F\right)\right)_{\mathbf{L}^{2}} .
\end{aligned}
$$

Как и выше, получим оценки

$$
\begin{gathered}
t^{2 \alpha-1} e^{\theta} \theta^{\prime} a_{0}\left(\left|\Phi+\left(g_{y}-\theta^{\prime} g\right) e^{-\theta}\right|^{\sigma}\left(\Phi+\left(g_{y}-\theta^{\prime} g\right) e^{-\theta}\right)-\Phi^{\sigma+1}\right. \\
\left.-(1+\sigma) \Phi^{\sigma}\left(g_{y}-\theta^{\prime} g\right) e^{-\theta}\right)=O\left(B t^{-1}\right), \\
t^{2 \alpha-1} \Phi_{y} B e^{\theta}\left(\left|\Phi+\left(g_{y}-\theta^{\prime} g\right) e^{-\theta}\right|^{\sigma}-\Phi^{\sigma}-\sigma \Phi^{\sigma-1}\left(g_{y}-\theta^{\prime} g\right) e^{-\theta}\right)=O\left(B t^{-1}\right), \\
t^{2 \alpha-1} B\left(\left(\Phi+\left(g_{y}-\theta^{\prime} g\right) e^{-\theta}\right)^{\sigma}-\Phi^{\sigma}\right)\left(2 \theta^{\prime} g_{y}-\left(\theta^{\prime 2}-\theta^{\prime \prime}\right) g\right)=O\left(t^{-1}\right)
\end{gathered}
$$

И

$$
\psi-\phi_{y}-t^{2 \alpha-1} B \Phi^{\sigma} \theta^{\prime}+t^{2 \alpha-1} \sigma B \Phi^{\sigma-1} \Phi_{y} \geqslant C B^{2} t^{2 \alpha-1}
$$

для всех $y \in \mathbb{R}$. Также имеем оценки

$$
\psi_{y}-t^{2 \alpha-1} B \Phi^{\sigma} \theta^{\prime 2}-t^{2 \alpha-1} \sigma B \Phi^{\sigma-1} \Phi_{y} \theta^{\prime}=O\left(B^{3} t^{2 \alpha-1}\right)
$$

и $\left(e^{\theta} F\right)_{y}=O\left(t^{-1}\right)$. Таким образом,

$$
\frac{d}{d t}\left\|G_{y}\right\|_{\mathbf{L}^{2}} \leqslant-t^{2 \alpha-1} C B^{2}\left\|G_{y}\right\|_{\mathbf{L}^{2}}+C B^{3} t^{2 \alpha-1}\|G\|_{\mathbf{L}^{2}}+C t^{-1} .
$$

Комбинируя это неравенство и (113), имеем

$$
\frac{d}{d t}\left(\left\|G_{y}\right\|_{\mathbf{L}^{2}}+B\|G\|_{\mathbf{L}^{2}}\right) \leqslant-C B^{2} t^{2 \alpha-1}\left(\left\|G_{y}\right\|_{\mathbf{L}^{2}}+B\|G\|_{\mathbf{L}^{2}}\right)+C t^{-1},
$$

откуда, интегрируя по времени, находим, что $\left\|G_{y}\right\|_{\mathbf{L}^{2}}^{2}+B\|G\|_{\mathbf{L}^{2}}^{2} \leqslant C t^{-2 \alpha}$ при всех $t \geqslant 0$. Аналогично оценим старшую производную: $\left\|G_{y y}\right\|_{\mathbf{L}^{2}}+B\left\|G_{y}\right\|_{\mathbf{L}^{2}}+$ $B^{2}\|G\|_{\mathbf{L}^{2}} \leqslant C t^{-2 \alpha}$ при всех $t \geqslant 0$. Результат теоремы теперь следует из оценки

$$
\begin{aligned}
|u(t, x)-\varphi(t, x) \Phi(y)| & \leqslant|u(t, x)-\varphi(t, x) \Phi(t, y)| \leqslant|\varphi(t, x)|\left|V_{y}(t, y)\right|+C t^{-\frac{1}{2}-\alpha} \\
& \leqslant|\varphi|\left|\left(g_{y}-\theta^{\prime} g\right) e^{-\theta}\right|+C t^{-\frac{1}{2}-\alpha} \leqslant C t^{-\frac{1}{2}-\alpha} .
\end{aligned}
$$

Теорема 23 доказана.

Мы глубоко признательны С. И. Похожаеву и С. Ю. Доброхотову за весьма ценные замечания и предложения.

\section{Список литературы}

[1] С. Л. Соболев, “Об одной новой задаче математической физики”, Изв. АН СССР. Сер. матем., 18:1 (1954), 3-50.

[2] Р.А. Александрян, "Спектральные свойства операторов, порожденных системой дифференциальных уравнений типа С. Л. Соболева", Тp. MMO, 9 (1980), 455-505. 
[3] С. А. Габов, Новые задачи математической теории волн, Физматлит, М., 1998.

[4] С. Ю. Доброхотов, "Нелокальные аналоги нелинейного уравнения Буссинеска для поверхностных волн над неровным дном и их асимптотические решения", Докл. АН СССР, 292:1 (1987), 63-67; англ. пер.: S. Yu. Dobrokhotov, "Nonlocal analogues of the nonlinear Boussinesq equation for surface waves over an uneven bottom and their asymptotic solutions", Soviet Phys. Dokl., 32:1 (1987), 18-20.

[5] Т. И. Зеленяк, Избранные вопросы качественной теории уравнений с частными производными, Спецкурс для студентов-математиков НГУ, Изд-во НГУ, Новосибирск, 1970.

[6] Б. В. Капитонов, "Теория потенциала для уравнения малых колебаний вращающейся жидкости", Матем. сб., 109:4 (1979), 607-628; англ. пер.: В. V. Kapitonov, "Potential theory for the equation of small oscillations of a rotating fluid", Math. USSR-Sb., 37:4 (1980), 559-579.

[7] В.Н. Масленникова, "Явные представления и априорные оценки решений граничных задач для систем Соболева", Сиб. матем. журн., 9:5 (1968), 1182-1198; англ. пер.: V. N. Maslennikova, "Explicit representations and a priori estimates for the solutions of boundary problems for Sobolev systems", Siberian Math. J., 9:5 (1968), 883-897.

[8] В.П. Маслов, "О существовании убывающего при $t \rightarrow \infty$ решения уравнения С. Л. Соболева для малых колебаний вращающейся жидкости в цилиндрической области", Сиб. матем. журн., 9:6 (1968), 1351-1359; англ. пер.: V.P. Maslov, "On the existence of a solution, decreasing as $t \rightarrow \infty$, of Sobolev's equation for small oscillations of a rotating fluid in a cylindrical domain", Siberian Math. J., 9:6 (1968), 1013-1020.

[9] Л.В. Овсянников, Н.И. Макаренко, В.Н.Налимов, В.Ю. Ляпидевский, П. И. Плотников, И. В. Штурова, В. И. Букреев, В. А. Владимиров, Нелинейные проблемы теории поверхностных и внутренних волн, Наука, Новосибирск, 1985.

[10] А.Г. Свешников, А.Б. Альшин, М. О. Корпусов, Ю. Д. Плетнер, Линейные и нелинейные уравнения соболевского типа, Физматлит, М., 2007.

[11] Г. И. Баренблатт, Ю.П. Желтов, И.Н. Кочина, "Об основных представлениях теории фильтрации в трещиноватых средах", ПМM, 24:5 (1960), 852-864; англ. пер.: G. I. Barenblatt, Iu. P. Zheltov, I. N. Kochina, "Basic concepts in the theory of seepage of homogeneous liquids in fissured rocks (strata)", J. Appl. Math. Mech., 24:5 (1960), 1286-1303.

[12] С.А. Габов, А. Г. Свешников, Линейные задачи теории нестационарных внутренних волн, Наука, М., 1990.

[13] С.Я. Секерж-Зенькович, "Построение фундаментального решения оператора внутренних волн”, Докл. АН СCCP, 246:2 (1979), 286-289; англ. пер.: S. Ja. Sekerž-Zen'kovič, "Fundamental solution of the interior wave operator", Soviet Phys. Dokl., 24:5 (1979), 347-349.

[14] М. О. Корпусов, А. Г. Свешников, "Разрушение решений абстрактных задач Коши для нелинейных дифференциально-операторных уравнений", Докл. $P A H$, 401:1 (2005), 12-15.

[15] J. Albert, "On the decay of solutions of the generalized Benjamin-Bona-Mahony equation", J. Math. Anal. Appl., 141:2 (1989), 527-537.

[16] J. W. Bebernes, A. A. Lacey, "Global existence and finite-time blow-up for a class of nonlocal parabolic problems", Adv. Differential Equations, 2:6 (1997), 927-953.

[17] T. B. Benjamin, J. L. Bona, J. J. Mahony, "Model equations for long waves in nonlinear dispersive systems", Philos. Trans. Roy. Soc. London Ser. A, 272:1220 (1972), $47-78$.

[18] P. Biler, "Long time behavior of the generalized Benjamin-Bona-Mahony equation in two space dimensions", Differential Integral Equations, 5:4 (1992), 891-901. 
[19] Y.M. Chen, "Remark on the global existence for the generalized Benjamin-BonaMahony equations in arbitrary dimension", Appl. Anal., 30:1-3 (1988), 1-15.

[20] T. Hagen, J. Turi, "On a class of nonlinear BBM-like equations", Comput. Appl. Math., 17:2 (1998), 161-172.

[21] G. Karch, "Large-time behavior of solutions to non-linear wave equations: higher-order asymptotics", Math. Methods Appl. Sci., 22:18 (1999), 1671-1697.

[22] L. Liu, M. Mei, "A better asymptotic profile of Rosenau-Burgers equation", Appl. Math. Comput., 131:1 (2002), 147-170.

[23] Ya. Liu, W. Wan, Sh. Lü, "Nonlinear pseudoparabolic equations in arbitrary dimensions", Acta Math. Appl. Sinica (English Ser.), 13:3 (1997), 265-278.

[24] L. A. Medeiros, M. G. Perla, "On global solutions of a nonlinear dispersive equation of Sobolev type", Bul. Soc. Bras. Math., 9:1 (1978), 49-59.

[25] M. Mei, "Long-time behavior of solution for Rosenau-Burgers equation. II", Appl. Anal., 68:3-4 (1998), 333-356.

[26] P. I. Naumkin, "Large-time asymptotic behaviour of a step for the BenjaminBona-Mahony-Burgers equation", Proc. Roy. Soc. Edinburgh Sect. A, 126:1 (1996), $1-18$.

[27] M. A. Park, "On the Rosenau equation in multidimensional space", Nonlinear Anal., 21:1 (1993), 77-85.

[28] J. M. Pereira, "Stability of multidimensional traveling waves for a BenjaminBona-Mahony type equation", Differential Integral Equations, 9:4 (1996), 849-863.

[29] L. Zhang, "Decay of solutions of generalized Benjamin-Bona-Mahony equations", Acta Math. Sinica (N.S.), 10:4 (1994), 428-438.

[30] Х. Гаевский, К. Грегер, К. Захариас, Нелинейные операторные уравнения и операторные дифференциальные уравнения, Мир, М., 1978; пер. с нем.: Н. Gajewski, K. Gröger, K. Zacharias, Nichtlineare Operatorgleichungen und Operatordifferentialgleichungen, Mathematische Lehrbucher und Monographien, II, Mathematische Monographien, 38, Akademie-Verlag, Berlin, 1974.

[31] Г. В. Демиденко, С.В. Успенский, Уравнения и системы, не разрешенные относительно старшей производной, Научная книга, Новосибирск, 1998; англ. пер.: G. V. Demidenko, S. V. Uspenskii, Partial differential equations and systems not solvable with respect to the highest-order derivative, Monogr. Textbooks Pure Appl. Math., 256, Dekker, New York, 2003.

[32] И.Е. Егоров, С. Г. Пятков, С. В. Попов, Неклассические дифференциально-операторные уравнения, Наука, Новосибирск, 2000.

[33] Г. А. Свиридюк, В. Е. Фёдоров, “Аналитические полугруппы с ядрами и линейные уравнения типа Соболева", Сиб. матем. журн., 36:5 (1995), 1130-1145; англ. пер.: G. A. Sviridyuk, V.E. Fedorov, "Analytic semigroups with kernels and linear equations of Sobolev type", Siberian Math. J., 36:5 (1995), 973-987.

[34] A. Favini, A. Yagi, Degenerate differential equations in Banach spaces, Monogr. Textbooks Pure Appl. Math., 215, Dekker, New York, 1999.

[35] U. Stefanelli, "On a class of doubly nonlinear nonlocal evolution equations", Differential Integral Equations, 15:8 (2002), 897-922.

[36] А.Л. Гладков, "Единственность решения задачи Коши для некоторых квазилинейных псевдопараболических уравнений", Матем. заметки, 60:3 (1996), 356-362; англ. пер.: A. L. Gladkov, "Unique solvability of the Cauchy problem for certain quasilinear pseudoparabolic equations", Math. Notes, 60:3 (1996), 264-268.

[37] А. И. Кожанов, "Начально-краевая задача для уравнений типа обобщенного уравнения Буссинеска с нелинейным источником", Матем. заметки, 65:1 (1999), 70-75; англ. пер.: A. I. Kozhanov, "Initial boundary value problem for generalized Boussinesq type equations with nonlinear source", Math. Notes, 65:1 (1999), 59-63. 
[38] С.И. Ляшко, Обобщенное управление линейными системами, Наукова думка, Киев, 1998; англ. пер.: S. I. Lyashko, Generalized optimal control of linear systems with distributed parameters, Appl. Optimization, 69, Kluwer, Dordrecht, 2002.

[39] Д. А. Номировский, “О гомеоморфизмах, осуществляемых некоторыми дифференциальными операторами с частными производными”, Укр. матем. журн., 2004, № 12, 1707-1716; англ. пер.: D. A. Nomirovskii, "On homeomorphisms realized by certain partial differential operators", Ukrainian Math. J., 56:12 (2005), 2017-2027.

[40] С. Г. Пятков, "Краевые задачи для некоторых уравнений и систем, возникающих в теории электрических цепей", Актуалъные проблемы современной математикu, 1, Изд-во НИИ МИОО НГУ, Новосибирск, 1995, 121-133.

[41] C. Guowang, W. Shubin, "Existence and non-existence of global solutions for nonlinear hyperbolic equations of higher order", Comment. Math. Univ. Carolin., 36:3 (1995), 475-487.

[42] H. Begehr, D. Q. Dai, "Initial boundary value problem for nonlinear pseudoparabolic equations", Complex Variables Theory Appl., 18:1-2 (1992), 33-47.

[43] E. Di Benedetto, Degenerate parabolic equations, Universitext, Springer-Verlag, New York, 1993.

[44] R. E. Showalter, Monotone operators in Banach space and nonlinear partial differential equations, Math. Surveys Monogr., 49, Amer. Math. Soc., Providence, RI, 1997.

[45] H. Fujita, "On the blowing up of solutions to the Cauchy problem for $u_{t}=\Delta u+u^{1+\alpha}$ ", J. Fac. Sci. Univ. Tokyo Sect. I, 13 (1966), 109-124.

[46] И.Е. Егоров, "Разрешимость одной краевой задачи для уравнения смешанного типа высокого порядка", Дифферени. уравнения, 23:9 (1987), 1560-1567; англ. пер.: I. E. Egorov, "Solvability of a boundary value problem for a high-order equation of mixed type", Differ. Equ., 23:9 (1987), 1075-1081.

[47] И. Е. Егоров, В. Е. Федоров, Неклассические уравнения математической физики высокого порядка, Изд-во ВЦ СО РАН, Новосибирск, 1995.

[48] Э. Митидиери, С. И. Похожаев, “Априорные оценки и отсутствие решений нелинейных уравнений и неравенств в частных производных", Тр. МИАН, 234, 2001, 1-383; англ. пер.: E. Mitidieri, S. I. Pokhozhaev, "A priori estimates and blow-up of solutions to nonlinear partial differential equations and inequalities", Proc. Steklov Inst. Math., 234 (2001), 1-362.

[49] А.А. Самарский, В.А. Галактионов, С.П. Курдюмов, А.П. Михайлов, Режимы с обострением в задачах для квазилинейных параболических уравнений, Наука, М., 1987; англ. пер.: А.А. Samarskii, V.A. Galaktionov, S.P. Kurdyumov, A.P. Mikhailov, Blow-up in quasilinear parabolic equations, de Gruyter Exp. Math., 19, de Gruyter, Berlin, 1995.

[50] H. Amann, M. Fila, "A Fujita-type theorem for the Laplace equation with a dynamical boundary condition", Acta Math. Univ. Comenian. (N.S.), 66:2 (1997), 321-328.

[51] S. Kaplan, "On the growth of solutions of quasi-linear parabolic equations", Comm. Pure Appl. Math., 16:3 (1963), 305-330.

[52] H. A. Levine, "Some nonexistence and instability theorems for solutions of the formally parabolic equations of the form $P u_{t}=-A u+F(u)$ ", Arch. Rational Mech. Anal., 51:5 (1973), 371-386.

[53] М. О. Корпусов, А. Г. Свешников, "О разрушении решений класса сильно нелинейных волновых диссипативных уравнений типа Соболева с источниками", Изв. РАН. Сер. матем., 69:4 (2005), 89-128; англ. пер.: М. О. Korpusov, A. G. Sveshnikov, "Blow-up of solutions of a class of strongly non-linear dissipative wave equations of Sobolev type with sources", Izv. Math., 69:4 (2005), 733-770.

[54] А. М. Ильин, "О поведении решения задачи Коши для параболического уравнения при неограниченном возрастании времени”, УМН, 16:2 (1961), 115-121. 
[55] C. J. Amick, J. L. Bona, M. E. Schonbek, "Decay of solutions of some nonlinear wave equations", J. Differential Equations, 81:1 (1989), 1-49.

[56] V. Bisognin, "On the asymptotic behavior of the solutions of a nonlinear dispersive system of Benjamin-Bona-Mahony's type", Boll. Un. Mat. Ital. B (7), 10:1 (1996), 99-128.

[57] J. L. Bona, L. Luo, "Decay of solutions to nonlinear dispersive wave equations", Differential Integral Equations, 6:5 (1993), 961-980.

[58] J. L. Bona, L. Luo, "More results on the decay of solutions to nonlinear, dispersive wave equations", Discrete Contin. Dynam. Systems, 1:2 (1995), 151-193.

[59] D. B. Dix, "The dissipation of nonlinear dispersive waves: the case of asymptotically weak nonlinearity", Comm. Partial Differential Equations, 17:9-10 (1992), 1665-1693.

[60] G. Karch, "Asymptotic behaviour of solutions to some pseudoparabolic equations", Math. Methods Appl. Sci., 20:3 (1997), 271-289.

[61] M. Mei, " $L_{q}$-decay rates of solutions for Benjamin-Bona-Mahony-Burgers equations", J. Differential Equations, 158:2 (1999), 314-340.

[62] M. Mei, C. Schmeiser, "Asymptotic profiles of solutions for the BBM-Burgers equations", Funkcial. Ekvac., 44:1 (2001), 151-170.

[63] R. Prado, E. Zuazua, "Asymptotic expansion for the generalized Benjamin-BonaMahony-Burgers equation”, Differential Integral Equations, 15:12 (2002), 1409-1434.

[64] Е.И. Кайкина, П. И. Наумкин, И. А. Шишмарев, “Задача Коши для уравнения типа Соболева со степенной нелинейностью", Изв. РАН. Сер. матем., 69:1 (2005), 61-114; англ. пер.: E. I. Kaikina, P. I. Naumkin, I. A. Shishmarev, "The Cauchy problem for an equation of Sobolev type with power non-linearity", Izv. Math., 69:1 (2005), 59-111.

[65] Е.И. Кайкина, П.И. Наумкин, И.А. Шишмарёв, "Асимптотика для уравнения типа Соболева с критической нелинейностью", Дифферени. уравнения, 43:5 (2007), 655-668; англ. пер.: E. I. Kaikina, P. I. Naumkin, I. A. Shishmarev, "Asymptotics for a Sobolev type equation with a critical nonlinearity", Differ. Equ., 43:5 (2007), 673-687.

[66] И. А. Шишмарёв, “Об одном нелинейном уравнении типа Соболева”, Дифферени. уравнения, 41:1 (2005), 138-140; англ. пер.: I. A. Shishmarev, "On a nonlinear Sobolev type equation", Differ. Equ., 41:1 (2005), 146-149.

[67] N. Hayashi, E. I. Kaikina, P. I. Naumkin, I. A. Shishmarev, Asymptotics for dissipative nonlinear equations, Lecture Notes in Math., 1884, Springer-Verlag, Berlin, 2006.

[68] S. Kamin, L. A. Peletier, "Large time behaviour of solutions of the heat equation with absorption", Ann. Scuola Norm. Sup. Pisa Cl. Sci. (4), 12:3 (1985), 393-408.

[69] В. А. Галактионов, С. П. Курдюмов, А. А. Самарский, "Об асимптотических "собственных функциях' задачи Коши для одного нелинейного параболического уравнения", Матем. сб., 126:4 (1985), 435-472; англ. пер.: V. A. Galaktionov, S.P. Kurdyumov, A. A. Samarskii, "On asymptotic 'eigenfunctions' of the Cauchy problem for a nonlinear parabolic equation", Math. USSR-Sb., 54:2 (1986), 421-455.

[70] M. Escobedo, O. Kavian, "Asymptotic behavior of positive solutions of a non-linear heat equation", Houston J. Math., 14:1 (1988), 39-50.

[71] M. Escobedo, O. Kavian, H. Matano, "Large time behavior of solutions of a dissipative semilinear heat equation", Comm. Partial Differential Equations, 20:7-8 (1994), $1427-1452$.

[72] A. Gmira, L. Veron, "Large time behavior of solutions of a semilinear parabolic equation in $R^{N} "$, J. Differential Equations, 53:2 (1984), 258-276.

[73] O. Kavian, "Remarks on the large time behavior of a nonlinear diffusion equation", Ann. Inst. H. Poincaré Anal. Non Linéaire, 4:5 (1987), 423-452. 
[74] E. Zuazua, "A dynamical system approach to the self-similar large time behavior in scalar convection-diffusion equation", J. Differential Equations, 108:1 (1994), 1-35.

[75] E. Zuazua, "Some recent results on the large time behavior for scalar parabolic conservation laws", Elliptic and parabolic problems (Pont-à-Mousson, 1994), Pitman Res. Notes Math. Ser., 325, Longman Sci. Tech., Harlow, 1995, 251-263.

[76] О. В. Бесов, В. П. Ильин, С. М. Никольский, Интегральные представления функиий и теоремы вложения, Наука, М., 1975; англ. пер.: О. V. Besov, V.P. Il'in, S. M. Nikol'skii, Integral representations of functions and imbedding theorems, Vol. I, Winston, Washington, DC; Wiley, New York-Toronto-London, 1978; O. V. Besov, V.P. Il'in, S. M. Nikol'skii, Integral representations of functions and imbedding theorems, Vol. II, Winston, Washington, DC; Wiley, New York-Toronto-London, 1979.

[77] Е. Стейн, Сингулярнье интеграль и дифференциальнье свойства функиий, Мир, M., 1973; пер. с англ.: Е. M. Stein, Singular integrals and differentiability properties of functions, Princeton Math. Ser., 30, Princeton Univ. Press, Princeton, NJ, 1970.

[78] Е. Титчмарш, Введение в теорию интегралов Фурье, Гостехиздат, М., Л., 1948; пер. с англ.: E. C. Titchmarsh, Introduction to the theory of Fourier integrals, Clarendon Press, Oxford, 1937.

[79] Дж. Ватсон, Теория Бесселевых функиий, ИЛ, М., 1949; пер. с англ.: G.N. Watson, A treatise on the theory of Bessel functions, Cambridge Univ. Press, Cambridge; Macmillan Company, New York, 1944.

[80] А.И. Кожанов, "Параболические уравнения с нелинейным нелокальным источником", Сиб. матем. журн., 35:5 (1994), 1062-1073; англ. пер.: А. I. Kozhanov, "Parabolic equations with nonlocal nonlinear source", Siberian Math. J., 35:5 (1994), 945-956.

[81] М. О. Корпусов, А. Г. Свешников, "Трехмерные нелинейные эволюционные уравнения псевдопараболического типа в задачах математической физики", ЖKBM $u$ $M \Phi, 43: 12$ (2003), 1835-1869; англ. пер.: M. O. Korpusov, A. G. Sveshnikov, "Threedimensional nonlinear evolution equations of pseudoparabolic type in problems of mathematical physics", Comput. Math. Math. Phys., 43:12 (2003), 1765-1797.

[82] M.E. Schonbek, "The Fourier splitting method", Advances in geometric analysis and continuum mechanics (Stanford, CA, 1993), Int. Press, Cambridge, MA, 1995, 269-274.

[83] N. Hayashi, E. I. Kaikina, P. I. Naumkin, "Large time behavior of solutions to dissipative nonlinear Schrödinger equation", Proc. Roy. Soc. Edinburgh Sect. A, 130:5 (2000), 1029-1043.

[84] A. Matsumura, K. Nishihara, "Asymptotics toward the rarefaction wave of the solutions of Burgers' equation with nonlinear degenerate viscosity", Nonlinear Anal., 23:5 (1994), 605-614.

\section{Е. И. Кайкина (Е. I. Kaikina)}

National Autonomous University of Mexico,

Institute of Mathematics

E-mail: ekaikina@matmor.unam.mx

\section{П. И. Наумкин (P. I. Naumkin)}

National Autonomous University of Mexico,

Institute of Mathematics

E-mail: pavelni@matmor.unam.mx

\section{И. А. Шишмарёв (I. A. Shishmarev)}

Московский государственный университет

им. М. В. Ломоносова

E-mail: shish@voxnet.ru
Поступила в редакцию 25.07 .2008 